TRANSACTIONS OF THE

AMERICAN MATHEMATICAL SOCIETY

Volume 364, Number 7, July 2012, Pages 3715-3749

S 0002-9947(2012)05604-2

Article electronically published on February 15, 2012

\title{
FINITE ORDER AUTOMORPHISMS ON REAL SIMPLE LIE ALGEBRAS
}

\author{
MENG-KIAT CHUAH
}

\begin{abstract}
We add extra data to the affine Dynkin diagrams to classify all the finite order automorphisms on real simple Lie algebras. As applications, we study the extensions of automorphisms on the maximal compact subalgebras and also study the fixed point sets of automorphisms.
\end{abstract}

\section{INTRODUCTION}

Let $\mathfrak{g}$ be a complex simple Lie algebra with Dynkin diagram D. A diagram automorphism on $\mathrm{D}$ of order $r$ leads to the affine Dynkin diagram $\mathrm{D}^{r}$. By assigning integers to the vertices, Kac [6] uses $\mathrm{D}^{r}$ to represent finite order $\mathfrak{g}$-automorphisms. This paper extends Kac's result. It uses $\mathrm{D}^{r}$ to represent finite order automorphisms on the real forms $\mathfrak{g}_{0} \subset \mathfrak{g}$. As applications, it studies the extensions of finite order automorphisms on the maximal compact subalgebras of $\mathfrak{g}_{0}$ and studies their fixed point sets. The special case of $\mathfrak{g}_{0}$-involutions (i.e. automorphisms of order 2) is discussed in [2].

In general, Gothic letters denote complex Lie algebras or spaces, and adding the subscript 0 denotes real forms. The finite order automorphisms on compact or complex simple Lie algebras have been classified by Kac, so we ignore these cases. Thus we always assume that $\mathfrak{g}_{0}$ is a noncompact real form of the complex simple Lie algebra $\mathfrak{g}$ (this automatically rules out complex $\mathfrak{g}_{0}$, since in this case $\mathfrak{g}$ has two simple factors).

The affine Dynkin diagrams are $\mathrm{D}^{1}$ for all $\mathrm{D}$, as well as $A_{n}^{2}, D_{n}^{2}, E_{6}^{2}$ and $D_{4}^{3}$. By Definition 1.1 below, we ignore $D_{4}^{3}$. We shall explain in (2.1) how $\mathrm{D}^{r}$ is related to the roots of $\mathfrak{g}$. These roots $\alpha$ are linearly dependent, and there are canonical positive integers $a_{\alpha}$ without nontrivial common factor such that

$$
\sum_{\mathrm{D}^{r}} a_{\alpha} \alpha=0
$$

[4. Ch. X-5], 7, Ch. 4]. For convenience, $\mathrm{D}^{r}$ denotes the diagram as well as its vertices, so we casually use the notation $\sum_{\mathrm{D}^{r}}$ or $\alpha \in \mathrm{D}^{r}$. We shall always let $a_{\alpha}$ be these canonical integers, known as the labels of $\mathrm{D}^{r}$.

Received by the editors October 4, 2010 and, in revised form, January 4, 2011.

2010 Mathematics Subject Classification. Primary 17B20, 17B22, 17B40, 20B25.

Key words and phrases. Real simple Lie algebra, Dynkin diagram, finite order automorphism.

This work was supported in part by the National Science Council of Taiwan.

(C)2012 American Mathematical Society Reverts to public domain 28 years from publication 
Let $\mathfrak{g}_{0}=\mathfrak{k}_{0}+\mathfrak{p}_{0}$ be a Cartan decomposition, namely $\mathfrak{k}_{0}$ is a maximal compact subalgebra of $\mathfrak{g}_{0}$ and $\mathfrak{p}_{0}$ is the orthogonal complement of $\mathfrak{k}_{0}$ under the Killing form.

Definition 1.1. A painted diagram is the diagram $\mathrm{D}^{r}$ such that each vertex has white or black color and $r \sum_{\text {black }} a_{\alpha}=2$. It is said to represent a real form $\mathfrak{g}_{0}=$ $\mathfrak{k}_{0}+\mathfrak{p}_{0}$ if $\mathfrak{k}$ has a Cartan subalgebra $\mathfrak{h}$ such that

(a) the root system $\Delta(\mathfrak{k}, \mathfrak{h})$ has a simple system represented by the white vertices so that the subdiagram of white vertices form the Dynkin diagram of $\mathfrak{k}$;

(b) the corresponding lowest weights of the adjoint $\mathfrak{k}$-representation on $\mathfrak{p}$ are represented by the black vertices.

Furthermore, the edges of the painted diagram are drawn according to the usual conditions of Dynkin diagrams.

Let aut(.) denote automorphisms, and int $(\cdot)$ denote inner automorphisms. The painted diagrams with underlying Dynkin diagrams $\mathrm{D}^{r}$ are denoted by $\mathrm{P}^{r}$. By Corollary 2.2, they correspond bijectively to the real forms of $\mathfrak{g}$ : If for $i=0,1, \mathrm{P}_{i}^{r}$ represents the real form $\mathfrak{g}_{i}$ of $\mathfrak{g}$, then $\mathrm{P}_{0}^{r}$ and $\mathrm{P}_{1}^{r}$ are related by a diagram symmetry if and only if $\mathfrak{g}_{0}$ and $\mathfrak{g}_{1}$ are isomorphic. If these conditions hold, the isomorphism $\phi: \mathfrak{g}_{0} \longrightarrow \mathfrak{g}_{1}$ extends naturally to aut $(\mathfrak{g})$. If we can choose $\phi$ which extends to $\operatorname{int}(\mathfrak{g})$, then we say that $\mathfrak{g}_{0}$ and $\mathfrak{g}_{1}$ are inner isomorphic. In Section 3 , we give a complete list of all painted diagrams and their corresponding real simple Lie algebras, up to isomorphism and inner isomorphism. Technical computations are used to distinguish isomorphic real forms which are not inner isomorphic, so they are left as an appendix in Section 10.

Due to Definition 1.1(a), let

$$
\mathrm{D}_{\mathfrak{k}}=\left\{\alpha \in \mathrm{P}^{r} ; \alpha \text { is white }\right\} .
$$

Here (1.2) denotes vertices as well as a subdiagram, where the edge relations of vertices of $D_{\mathfrak{k}}$ are the same as their edge relations in $\mathrm{P}^{r}$. Strictly speaking, $\mathrm{D}_{\mathfrak{k}}$ is the Dynkin diagram of $[\mathfrak{k}, \mathfrak{k}]$, as $\mathfrak{k}$ may have a nontrivial center (see (2.2) ).

Let $U$ be the multiplicative group of complex numbers of finite order, namely

$$
U=\left\{z \in \mathbb{C} ; z^{m}=1 \text { for some } m \in \mathbb{N}\right\} .
$$

In the following, $c: \mathrm{P}^{r} \longrightarrow U$ means $c_{\alpha} \in U$ for all vertices $\alpha \in \mathrm{P}^{r}$. The diagram automorphism $d \in \operatorname{aut}\left(\mathrm{P}^{r}\right)$ is always understood to preserve vertex colors.

Let $\mathfrak{g}_{\alpha}$ be the root spaces of $\alpha \in \Delta(\mathfrak{g}, \mathfrak{h})$. If $r=1$, then $\mathfrak{h}$ is a Cartan subalgebra of $\mathfrak{g}$, so $\operatorname{dim} \mathfrak{g}_{\alpha}=1$. But if $r=2$, then $\operatorname{rank} \mathfrak{g}>\operatorname{dim} \mathfrak{h}$ and we may have $\operatorname{dim} \mathfrak{g}_{\alpha}=2$, where $\mathfrak{g}_{\alpha}=\left(\mathfrak{g}_{\alpha} \cap \mathfrak{k}\right)+\left(\mathfrak{g}_{\alpha} \cap \mathfrak{p}\right)$ is a decomposition into 1-dimensional subspaces. Given $\alpha \in \mathrm{P}^{r}$, we define $\mathfrak{g}_{\alpha}^{1}=\mathfrak{g}_{\alpha} \cap \mathfrak{k}$ if $\alpha$ is white and $\mathfrak{g}_{\alpha}^{1}=\mathfrak{g}_{\alpha} \cap \mathfrak{p}$ if $\alpha$ is black. Then $\operatorname{dim} \mathfrak{g}_{\alpha}^{1}=1$ for all $\alpha \in \mathrm{P}^{r}$.

Definition 1.2. Let $\mathrm{P}^{r}$ represent $\mathfrak{g}_{0}$. A marking on $\mathrm{P}^{r}$ is a pair $(c, d)$, where

$$
c: \mathrm{P}^{r} \longrightarrow U, d \in \operatorname{aut}\left(\mathrm{P}^{r}\right) .
$$

It is said to represent a finite order $\mathfrak{g}_{0}$-automorphism $\sigma$ if there exist vector space automorphisms $\sigma_{c}, \sigma_{d}$ on $\sum_{\alpha \in \mathrm{P}^{r}} \mathfrak{g}_{\alpha}^{1}$ and basis $\left\{X_{\alpha} \in \mathfrak{g}_{\alpha}^{1}\right\}_{\alpha \in \mathrm{P}^{r}}$ such that

(a) $\sigma_{c}$ acts as multiplication by $c_{\alpha}$ on $\mathfrak{g}_{\alpha}^{1}$;

(b) $\sigma_{d} X_{\alpha}=X_{d \alpha}$ for all $\alpha \in \mathrm{P}^{r}$;

(c) $\sigma=\sigma_{c} \cdot \sigma_{d}$ on $\sum_{\alpha \in \mathrm{P}^{r}} \mathfrak{g}_{\alpha}^{1}$. 
Not all markings represent an automorphism; we next look for the ones which do. Given $d \in \operatorname{aut}\left(\mathrm{P}^{r}\right)$, a pair of adjacent vertices $\{\gamma, \delta\}$ such that $d \gamma=\delta$ and $d \delta=\gamma$ is called a special orbit of $d$. Let $S$ be the set of all special orbits, namely

$$
S=\{\{\gamma, \delta\} \text { adjacent; } d \gamma=\delta, d \delta=\gamma\}
$$

If $\beta=\{\gamma, \delta\}$ is a $d$-orbit, then $a_{\gamma}=a_{\delta}$, so we can define $a_{\beta}=a_{\gamma}=a_{\delta}$. We say that a marking $(c, d)$ on $\mathrm{P}^{r}$ is admissible if

$$
\Pi_{\beta \in S}(-1)^{a_{\beta}} \Pi_{\alpha \in \mathrm{P}^{r}} c_{\alpha}^{r a_{\alpha}}=1 .
$$

Let $\mathrm{lcm}$ denote the least common multiple, and let ord denote the order. If $(c, d)$ is admissible, we define its order by

$$
\operatorname{ord}(c, d)=\operatorname{lcm}\left\{|\mathcal{O}| \operatorname{ord}\left(\Pi_{\alpha \in \mathcal{O}} c_{\alpha}\right) ; \mathcal{O} \subset \mathrm{P}^{r} \text { is a } d \text {-orbit }\right\}
$$

where $|\mathcal{O}|$ is the number of vertices in $\mathcal{O}$. The following is the main theorem of this article.

Theorem 1.3. Let $\mathrm{P}^{r}$ represent $\mathfrak{g}_{0}$. An admissible marking $(c, d)$ on $\mathrm{P}^{r}$ represents a unique finite order $\mathfrak{g}_{0}$-automorphism $\sigma$, and $\operatorname{ord}(\sigma)=\operatorname{ord}(c, d)$. Conversely, every finite order $\mathfrak{g}_{0}$-automorphism is represented by an admissible marking on $\mathrm{P}^{r}$.

In this theorem, uniqueness of $\sigma$ is understood to be up to its conjugate class in $\operatorname{aut}\left(\mathfrak{g}_{0}\right)$. The theorem says that every admissible marking on $\mathrm{P}^{r}$ represents a unique finite order $\mathfrak{g}_{0}$-automorphism. But conversely, a $\mathfrak{g}_{0}$-automorphism may be represented by several admissible markings. In view of this, we shall introduce the notions of equivalent and inner equivalent admissible markings in Definition 5.3. and prove the following theorem.

Theorem 1.4. Let $(c, d),\left(c^{\prime}, d^{\prime}\right)$ be admissible markings on $\mathrm{P}^{r}$ which represent $\sigma, \sigma^{\prime} \in \operatorname{aut}\left(\mathfrak{g}_{0}\right)$, respectively. Then $(c, d)$ and $\left(c^{\prime}, d^{\prime}\right)$ are equivalent (resp. inner equivalent) if and only if $\sigma$ and $\sigma^{\prime}$ are conjugate by aut $\left(\mathfrak{g}_{0}\right)\left(r e s p\right.$. $\left.\operatorname{int}\left(\mathfrak{g}_{0}\right)\right)$.

Theorems 1.3 and 1.4 lead to a bijective correspondence between the equivalence (resp. inner equivalence) classes of admissible markings on $\mathrm{P}^{r}$ and the finite order $\mathfrak{g}_{0}$-automorphisms up to conjugation by $\operatorname{aut}\left(\mathfrak{g}_{0}\right)\left(\operatorname{resp} \operatorname{int}\left(\mathfrak{g}_{0}\right)\right)$.

Next we provide two applications of Theorem 1.3. The first application studies the extensions of finite order $\mathfrak{k}_{0}$-automorphisms to $\mathfrak{g}_{0}$, as given by Corollaries 1.5 and 1.6. The second application finds the fixed point sets of $\mathfrak{g}_{0}$-automorphisms, as given by Theorem 1.7 .

We say that $\mathfrak{g}_{0}$ is of Hermitian type if the Riemannian symmetric space [4] of $\mathfrak{g}_{0}$ has an invariant Hermitian structure. We say that $\mathfrak{g}_{0}$ is of equal rank type if $\mathfrak{g}_{0}$ and $\mathfrak{k}_{0}$ have equal rank. There are three cases of $\mathfrak{g}_{0}$ :

$$
\begin{aligned}
& \text { (a) Hermitian type (automatically equal rank type); } \\
& \text { (b) non-Hermitian and equal rank type; } \\
& \text { (c) non-Hermitian and nonequal rank type. }
\end{aligned}
$$

These cases are revealed by $\mathrm{P}^{r}$ in (2.2).

Automorphisms which are not inner are said to be outer. We write aut $\left(\mathfrak{k}_{0}\right)=$ $\operatorname{int}\left(\mathfrak{k}_{0}\right) \cup \operatorname{out}\left(\mathfrak{k}_{0}\right)$ accordingly. Write $\mathfrak{k}_{0}=\left[\mathfrak{k}_{0}, \mathfrak{k}_{0}\right]+\mathfrak{z}_{0}$, where $\left[\mathfrak{k}_{0}, \mathfrak{k}_{0}\right]$ is semisimple and $\mathfrak{z}_{0}$ is the center of $\mathfrak{k}_{0}$. For $\mathfrak{g}_{0}$ of Hermitian type $(1.6)(\mathrm{a}), \operatorname{dim} \mathfrak{z}_{0}=1$. So if $\sigma \in \operatorname{out}\left(\mathfrak{k}_{0}\right)$, it may act as an outer automorphism on $\left[\mathfrak{k}_{0}, \mathfrak{k}_{0}\right]$ or $\mathfrak{z}_{0}$.

Every finite order $\mathfrak{g}_{0}$-automorphism preserves a Cartan decomposition $\mathfrak{g}_{0}=\mathfrak{k}_{0}+$ $\mathfrak{p}_{0}$, so it can be regarded as an extension from $\mathfrak{k}_{0}$. Let aut ${ }_{n}(\cdot)$ denote automorphisms 
of order $n$, and let $\sigma \in \operatorname{aut}_{n}\left(\mathfrak{k}_{0}\right)$. By Corollary 4.3, $\sigma$ can be represented by a marking $(c, d)$ on the white vertices $\mathrm{D}_{\mathfrak{k}}$, namely $c: \mathrm{D}_{\mathfrak{k}} \longrightarrow U$ and $d \in \operatorname{aut}\left(\mathrm{D}_{\mathfrak{k}}\right)$. The next two corollaries study the extension of $\sigma$ to aut ${ }_{m}\left(\mathfrak{g}_{0}\right)$. It is necessary that $n$ divides $m$ and that $d$ extends to aut $\left(\mathrm{P}^{r}\right)$. Similar to (1.4), let

$$
S_{\mathfrak{k}}=\left\{\text { special } d \text {-orbits in } \mathrm{D}_{\mathfrak{k}}\right\} \subset S \text {. }
$$

Define

$$
w=\Pi_{\beta \in S_{\mathfrak{k}}}(-1)^{a_{\beta}} \Pi_{\alpha \in \mathrm{D}_{\mathfrak{k}}} c_{\alpha}^{r a_{\alpha}} .
$$

Corollary 1.5. Let $\mathfrak{g}_{0}$ be of Hermitian type. Let $\sigma \in \operatorname{aut}_{n}\left(\mathfrak{k}_{0}\right)$ be represented by a marking $(c, d)$ on $\mathrm{D}_{\mathfrak{k}}$, and suppose that d extends to aut $\left(\mathrm{P}^{1}\right)$.

(a) $\sigma \in \operatorname{int}\left(\mathfrak{k}_{0}\right): \sigma$ extends to aut $_{q n}\left(\mathfrak{g}_{0}\right)$ for all $q \in \mathbb{N}$.

(b) $\sigma \in \operatorname{int}\left(\left[\mathfrak{k}_{0}, \mathfrak{k}_{0}\right]\right) \oplus \operatorname{out}\left(\mathfrak{z}_{0}\right):$ For $\mathfrak{s u}(2,2), \mathfrak{s p}(2, \mathbb{R})$ and $\mathfrak{s o}(2, q)$, $\sigma$ extends to aut $_{\operatorname{lcm}(n, 2 \operatorname{ord}(w))}\left(\mathfrak{g}_{0}\right)$. In all other cases, $\sigma$ does not extend to $\mathfrak{g}_{0}$.

(c) $\sigma \in \operatorname{out}\left(\left[\mathfrak{k}_{0}, \mathfrak{k}_{0}\right]\right) \oplus \operatorname{int}\left(\mathfrak{z}_{0}\right):$ For $\mathfrak{s u}(p, p)$ and $\mathfrak{s o}(2,2 q), \sigma$ extends to $\operatorname{aut}_{q n}\left(\mathfrak{g}_{0}\right)$ for all $q \in \mathbb{N}$. In all other cases, $\sigma$ does not extend to $\mathfrak{g}_{0}$.

(d) $\sigma \in \operatorname{out}\left(\left[\mathfrak{k}_{0}, \mathfrak{k}_{0}\right]\right) \oplus \operatorname{out}\left(\mathfrak{z}_{0}\right):$ For $\mathfrak{s u}(1, q), \sigma$ extends to $\operatorname{aut}_{\operatorname{lcm}(n, 2 \operatorname{ord}(-w))}\left(\mathfrak{g}_{0}\right)$. In all other cases, $\sigma$ extends to aut $\operatorname{acm}_{\operatorname{lcm}(n \operatorname{ord}(w))}\left(\mathfrak{g}_{0}\right)$.

Corollary 1.6. Let $\mathfrak{g}_{0}$ be of non-Hermitian type, and $\sigma \in \operatorname{aut}_{n}\left(\mathfrak{k}_{0}\right)$ be represented by a marking $(c, d)$ on $\mathrm{D}_{\mathfrak{k}}$. Then $\sigma$ extends to aut $\left(\mathfrak{g}_{0}\right)$ if and only if $d$ extends to aut $\left(\mathrm{P}^{r}\right)$. In that case $\sigma$ has exactly two extensions, and they belong to

$$
\text { aut }_{\operatorname{lcm}\{n, \operatorname{ord}( \pm \sqrt{w})\}}\left(\mathfrak{g}_{0}\right) \subset \operatorname{aut}_{n}\left(\mathfrak{g}_{0}\right) \cup \operatorname{aut}_{2 n}\left(\mathfrak{g}_{0}\right) .
$$

As explained in Remark 8.1 for $\mathrm{P}^{2}$ the two extensions of $\sigma$ to $\mathfrak{g}_{0}$ are not conjugate. But for $\mathrm{P}^{1}$ different extensions of $\sigma$ to $\mathfrak{g}_{0}$ may or may not be conjugate. They are judged by Theorem 1.4 .

Consider the markings $(c, d)$ on $\mathrm{P}^{r}$ of the form

$$
d=1, \quad \Pi_{\mathrm{P}^{1}} c_{\alpha}^{a_{\alpha}}=1 \text { or } \Pi_{\mathrm{P}^{2}} c_{\alpha}^{a_{\alpha}}=-1 .
$$

By (1.5) they are admissible, and so they represent finite order $\mathfrak{g}_{0}$-automorphisms $\sigma$. We shall explain in Proposition 8.2 that for markings in (1.8), $\sigma$ is inner for $\mathrm{P}^{1}$ and outer for $\mathrm{P}^{2}$. Another application of Theorem 1.3 is to find their fixed point sets

$$
\mathfrak{g}_{0}^{\sigma}=\left\{X \in \mathfrak{g}_{0} ; \sigma X=X\right\} .
$$

We shall construct two Dynkin diagrams $\mathrm{D}\left(\sigma_{\mathfrak{g}}\right)$ and $\mathrm{D}\left(\sigma_{\mathfrak{k}}\right)$ in (8.3) and (8.6), as well as two nonnegative integers $p$ and $q$ in (8.4) and (8.7). They describe $\mathfrak{g}_{0}^{\sigma}$ by the following theorem.

Theorem 1.7. Suppose that $\sigma$ is represented by a marking in (1.8). Then $\mathfrak{g}_{0}^{\sigma}$ is the real reductive Lie algebra such that

(a) $\mathfrak{g}^{\sigma}$ has Dynkin diagram $\mathrm{D}\left(\sigma_{\mathfrak{g}}\right)$ and center $\mathbb{C}^{p}$;

(b) $\mathfrak{k}^{\sigma}$ has Dynkin diagram $\mathrm{D}\left(\sigma_{\mathfrak{k}}\right)$ and center $\mathbb{C}^{q}$;

(c) rank $\mathfrak{g}^{\sigma}=\operatorname{rank} \mathfrak{k}^{\sigma}$, so the center of $\mathfrak{g}_{0}^{\sigma}$ is compact.

Although $\mathfrak{g}_{0}^{\sigma}$ may have a nontrivial center, the Killing form of $\mathfrak{g}_{0}$ provides the notion of compactness on $\mathfrak{g}_{0}^{\sigma}$. So in Theorem 1.7(b), (c), the maximal compact subalgebra of $\mathfrak{g}_{0}^{\sigma}$ is defined by $\mathfrak{k}_{0}^{\sigma}=\mathfrak{k}_{0} \cap \mathfrak{g}_{0}^{\sigma}$.

Theorem 1.7 provides an effective method to compute $\mathfrak{g}_{0}^{\sigma}$. In general, a real simple Lie algebra is determined by its complexification and its maximal compact subalgebra [4, Ch. X-6, Thm. 6.2]. Here $\mathfrak{g}_{0}^{\sigma}$ is real reductive, so the presence of 
several simple ideals and centers makes it harder to determine $\mathfrak{g}_{0}^{\sigma}$ solely by $\mathfrak{g}^{\sigma}$ and $\mathfrak{k}^{\sigma}$. However, since we also have rank $\mathfrak{g}^{\sigma}=\operatorname{rank} \mathfrak{k}^{\sigma}$, the theorem essentially determines $\mathfrak{g}_{0}^{\sigma}$.

We shall provide three examples, Examples 9.1, 9.2 and 9.3, to illustrate the above ideas. They show how to find $\operatorname{aut}_{m}\left(\mathfrak{g}_{0}\right)$ up to conjugation by automorphisms, to find the extensions of $\operatorname{aut}_{n}\left(\mathfrak{k}_{0}\right)$ to aut ${ }_{m}\left(\mathfrak{g}_{0}\right)$, to find the fixed point sets $\mathfrak{g}_{0}^{\sigma}$, and to find the outer automorphisms.

This paper is organized as follows. In Section 2, we provide some preliminary facts about Lie algebras. Section 3 provides the list of all the real forms $\mathfrak{g}_{0}$ with their painted diagrams up to isomorphism and inner isomorphism. In Section 4, we prove Theorem 1.3, which shows that the admissible markings represent finite order $\mathfrak{g}_{0}$-automorphisms. In Section 5, we prove Theorem 1.4 which judges markings for conjugate $\mathfrak{g}_{0}$-automorphisms. To study the extensions of finite order $\mathfrak{k}_{0}$-automorphisms, we prove Corollary 1.5 in Section 6 and prove Corollary 1.6 in Section 7. In Section 8, we study the fixed point sets and prove Theorem 1.7. In Section 9, we give some examples to illustrate the above ideas. In Section 10, we provide the computations which distinguish the isomorphic real forms that are not inner isomorphic, leading to Figure 4 of Section 3.

\section{Preliminaries on Simple Lie algebras}

In this section, we provide some basic properties of Lie algebras which will be used later. Kac's fundamental theorem (Theorem 2.1) first appears as a brief announcement in [6] and later as a detailed exposition in [4, Ch. X-5] and [7, Ch. 8]. Let $\mathfrak{g}$ be a complex simple Lie algebra with Cartan subalgebra $\tilde{\mathfrak{h}}$. A simple system $\Pi_{\mathfrak{g}}$ of $\Delta(\mathfrak{g}, \tilde{\mathfrak{h}})$ leads to the Dynkin diagram D whose vertices represent $\Pi_{\mathfrak{g}}$. Consider a diagram symmetry $d$ on $\mathrm{D}$ of order $r \in\{1,2\}$ (we ignore $r=3$ due to Definition 1.11). It lifts to an $\tilde{\mathfrak{h}}$-stable $\mathfrak{g}$-automorphism of order $r$ whose eigenspaces form a $\mathbb{Z}_{r}$-grading $\mathfrak{g}=\sum_{i \in \mathbb{Z}_{r}} \mathfrak{g}^{i}$, and $\mathfrak{h}=\tilde{\mathfrak{h}} \cap \mathfrak{g}^{\overline{0}}$ is a Cartan subalgebra of $\mathfrak{g}^{\overline{0}}$. Let $\Pi_{\overline{0}} \subset \Delta\left(\mathfrak{g}^{\overline{0}}, \mathfrak{h}\right)$ be the simple system and root system of $\mathfrak{g}^{\overline{0}}$, obtained by restricting $\Pi_{\mathfrak{g}} \subset \Delta(\mathfrak{g}, \tilde{\mathfrak{h}})$ to $\mathfrak{h}$. Construct $\mathrm{D}^{r}$ by letting its vertices be

$$
\mathrm{D}^{r}=\Pi_{\overline{0}} \cup\left\{\text { lowest weight of the adjoint } \mathfrak{g}^{\overline{0}} \text {-representation on } \mathfrak{g}^{\overline{1}}\right\} \text {. }
$$

Also, adjacent vertices of $\mathrm{D}^{r}$ correspond to the simple rank two subalgebra indicated by the subdiagram (except for $A_{1}^{1}$ ). For $r=1$, we have $\mathfrak{g}=\mathfrak{g}^{\overline{0}}=\mathfrak{g}^{\overline{1}}$ and $\mathfrak{h}=\tilde{\mathfrak{h}}$, so $\mathrm{D}^{1}$ is just the extended Dynkin diagram $\Pi_{\mathfrak{g}} \cup\{$ lowest root $\}$. For $r=2$, the subdiagram $\Pi_{\overline{0}} \subset \mathrm{D}^{2}$ consists of the $d$-orbits of vertices of $\Pi_{\mathfrak{g}}$, and the edges of $\Pi_{\overline{0}}$ are drawn according to the root lengths

$$
\begin{aligned}
\text { adjacent } 2 \text {-element } d \text {-orbit } & <\text { nonadjacent } 2 \text {-element } d \text {-orbit } \\
& <\text { vertices fixed by } d .
\end{aligned}
$$

In each case of $\mathrm{D}^{r}$, we have added one extra vertex to $\Pi$, so $\sharp\left(\mathrm{D}^{r}\right)=\operatorname{dim} \mathfrak{h}+1$. Hence there is a nontrivial linear combination $\sum_{\mathrm{D}^{r}} a_{\alpha} \alpha=0$, and the coefficients $\left\{a_{\alpha}\right\}_{\mathrm{D}^{r}}$ in (1.1) are the unique ones which are positive integers without nontrivial common factor.

Theorem 2.1 (Kac). Let $\left\{n_{\alpha}\right\}_{\mathrm{D}^{r}}$ be nonnegative integers without nontrivial common factor, let $m=r \sum_{\mathrm{D}^{r}} a_{\alpha} n_{\alpha}$, and let $c_{\alpha}=\left(\exp \frac{2 \pi i}{m}\right)^{n_{\alpha}}$. Fix $\left\{X_{\alpha}\right\}_{\mathrm{D}^{r}}$ from the simple root vectors of $\mathfrak{g}^{\overline{0}}$ and a lowest root vector of $\mathfrak{g}^{\overline{1}}$. Then $\left\{X_{\alpha}\right\}_{\mathrm{D}^{r}}$ generate $\mathfrak{g}$. 
(a) The data $\left\{c_{\alpha}\right\}_{\mathrm{D}^{r}}$ represents a unique $\mathfrak{g}$-automorphism $\sigma$ of order $m$ by $\sigma X_{\alpha}=$ $c_{\alpha} X_{\alpha}$ for all $\alpha \in \mathrm{D}^{r}$.

(b) The fixed point set of $\sigma$ is a reductive algebra $\mathfrak{g}^{\sigma}=\left[\mathfrak{g}^{\sigma}, \mathfrak{g}^{\sigma}\right]+Z\left(\mathfrak{g}^{\sigma}\right)$. The Dynkin diagram of the semisimple subalgebra $\left[\mathfrak{g}^{\sigma}, \mathfrak{g}^{\sigma}\right]$ is the subdiagram $\left\{\alpha \in \mathrm{D}^{r} ; c_{\alpha}=1\right\}$. The dimension of the center $Z\left(\mathfrak{g}^{\sigma}\right)$ is $\sharp\left\{\alpha \in \mathrm{D}^{r} ; c_{\alpha} \neq 1\right\}-1$.

(c) Every finite order $\mathfrak{g}$-automorphism can be represented uniquely in this way.

The uniqueness of $\sigma$ in Theorem 2.1(a) is up to conjugate class in aut( $\mathfrak{g})$. In Theorem 2.1(b), $\sharp(\cdot)$ denotes the number of vertices. The uniqueness of $\left\{n_{\alpha}\right\}_{\mathrm{D}^{r}}$ in Theorem 2.1 (c) is up to diagram symmetry.

Set $m=2$ in Theorem 2.1. Then $c_{\alpha}= \pm 1$ for all $\alpha \in \mathrm{D}^{r}$. We can paint $\alpha$ as white if $c_{\alpha}=1$ and paint $\alpha$ as black if $c_{\alpha}=-1$. This leads to the painted diagram $\mathrm{P}^{r}$ of Definition 1.1. By Theorem 2.1(a), $\mathrm{P}^{r}$ represents a $\mathfrak{g}$-involution $\theta$, where $\theta=1$ on the root spaces of white vertices and $\theta=-1$ on the root spaces of black vertices. The $\mathfrak{g}$-involutions $\theta$ correspond to the real forms $\mathfrak{g}_{0}$ of $\mathfrak{g}$, given by $\theta$ which preserves $\mathfrak{g}_{0}$ and restricts to a Cartan involution on $\mathfrak{g}_{0}$. This leads to the following corollary. The painted diagrams and real forms are listed in Section 3.

Corollary 2.2. There is a bijective correspondence between the painted diagrams (up to diagram symmetry) and the real forms of $\mathfrak{g}$ (up to isomorphism).

By Definition 1.1, the condition $r \sum_{\text {black }} a_{\alpha}=2$ produces three cases of painted diagrams $\mathrm{P}^{r}$. They correspond to the three cases (1.6) of $\mathfrak{g}_{0}$ as follows:

$$
\begin{aligned}
& \text { (a) } r=1 \text {, two black vertices } \gamma, \delta \text { with } a_{\gamma}=a_{\delta}=1 \\
& \Longleftrightarrow \mathfrak{g}_{0} \text { is of Hermitian type; } \\
& \text { (b) } r=1 \text {, one black vertex } \gamma \text { with } a_{\gamma}=2 \\
& \Longleftrightarrow \mathfrak{g}_{0} \text { is of non-Hermitian and equal rank type; } \\
& \text { (c) } r=2 \text {, one black vertex } \gamma \text { with } a_{\gamma}=1 \\
& \Longleftrightarrow \mathfrak{g}_{0} \text { is of non-Hermitian and nonequal rank type. }
\end{aligned}
$$

The Cartan involution $\theta$ leads to a Cartan decomposition $\mathfrak{g}_{0}=\mathfrak{k}_{0}+\mathfrak{p}_{0}$, where $\mathfrak{k}_{0}$ and $\mathfrak{p}_{0}$ are the \pm 1 -eigenspaces of $\theta$. Write $\mathfrak{k}_{0}=\left[\mathfrak{k}_{0}, \mathfrak{k}_{0}\right]+\mathfrak{z}_{0}$. By Theorem 2.1(b), the white vertices form the Dynkin diagram of $\left[\mathfrak{k}_{0}, \mathfrak{k}_{0}\right]$, denoted by $\mathrm{D}_{\mathfrak{k}}$. Theorem 2.1(b) also says that $\operatorname{dim} \mathfrak{z}_{0}=1$ in (2.2) (a), and $\mathfrak{z}_{0}=0$ in (2.2) (b), (c). By Definition 1.1(b), based on the number of black vertices, $\mathfrak{p}$ has two irreducible components in (2.2) (a), and $\mathfrak{p}$ is irreducible in (2.2) (b), (c).

In the following proposition and corollary, we shall show that finite order $\mathfrak{g}_{0^{-}}$ automorphisms can be identified with finite order $\mathfrak{g}$-automorphisms which commute with $\theta$.

Proposition 2.3. Let $G$ be a connected real semisimple Lie group, and let $\Gamma \subset$ $\operatorname{aut}(G)$ be a finite subgroup. Then $G$ has a $\Gamma$-stable maximal compact subgroup.

Proof. Let 1 denote the identity element of $G$ or $\Gamma$. Since $\Gamma$ acts on $G$, we can form the semidirect product

$$
\tilde{G}=G \rtimes \Gamma
$$

with normal subgroup $(G, 1)$. Identify $G$ with $(G, 1)$ and write $G \subset \tilde{G}$. Similarly, identify $\Gamma$ with $(1, \Gamma)$ and write $\Gamma \subset \tilde{G}$. Since $\Gamma$ is finite, it is a compact subgroup of $\tilde{G}$. By [10, Thm. 3.1], $\Gamma$ must be contained in a maximal compact subgroup $\tilde{K}$ of $\tilde{G}$. Let

$$
K=\tilde{K} \cap G
$$


We now show that $K$ is a $\Gamma$-stable maximal compact subgroup of $G$, which proves the proposition.

We first show that $K$ is $\Gamma$-stable. Pick $k \in K$ and $\gamma \in \Gamma$, and we need to check that $\gamma k \in K$. Here $(1, \gamma),\left(1, \gamma^{-1}\right) \in \Gamma \subset \tilde{K}$ and $(k, 1) \in K \subset \tilde{K}$, hence $(1, \gamma)(k, 1)\left(1, \gamma^{-1}\right) \in \tilde{K}$. By the group law of a semidirect product, $(1, \gamma)(k, 1)\left(1, \gamma^{-1}\right)$ $=(\gamma k, 1) \in G$. We conclude that $\gamma k \in \tilde{K} \cap G=K$. Hence $K$ is $\Gamma$-stable.

It remains to show that $K$ is a maximal compact subgroup of $G$. Suppose otherwise, namely there exists a maximal compact subgroup $U$ of $G$ such that $K \subset U \subset G$ and $K \neq U$. Since $G$ is connected, so is $U$. Hence $\operatorname{dim} K<\operatorname{dim} U$. Let $\tilde{U}$ be a maximal compact subgroup of $\tilde{G}$ which contains $U$. Then $\operatorname{dim} U \leq \operatorname{dim} \tilde{U}$. Since $\tilde{G}$ has finitely many connected components, its maximal compact subgroups $\tilde{K}$ and $\tilde{U}$ are conjugate [10, Thm. 3.1]. Hence $\operatorname{dim} \tilde{K}=\operatorname{dim} \tilde{U}$. We have obtained

$$
\operatorname{dim} K<\operatorname{dim} U \leq \operatorname{dim} \tilde{U}=\operatorname{dim} \tilde{K} .
$$

Since $K=\tilde{K} \cap G$ and since $G$ is a connected component of $\tilde{G}$, it follows that $\operatorname{dim} K=\operatorname{dim} \tilde{K}$. Hence (2.3) is a contradiction. We conclude that $K$ is a maximal compact subgroup of $G$.

\section{Corollary 2.4.}

(a) Given a finite subgroup $\Gamma \subset$ aut $\left(\mathfrak{g}_{0}\right)$, there exists a Cartan involution $\theta$ on $\mathfrak{g}_{0}$ which centralizes $\Gamma$. Furthermore, $\theta$ is unique up to conjugation by an inner $\mathfrak{g}_{0}$-automorphism which centralizes $\Gamma$.

(b) Let $\theta$ and $\sigma$ be commuting $\mathfrak{g}$-automorphisms, where $\operatorname{ord}(\theta)=2$ and $\operatorname{ord}(\sigma)<$ $\infty$. Then there exists a $\theta$-stable and $\sigma$-stable real form $\mathfrak{g}_{0} \subset \mathfrak{g}$ such that $\theta$ is a Cartan involution on $\mathfrak{g}_{0}$.

Proof. Let $G=\operatorname{int}\left(\mathfrak{g}_{0}\right)$. Then $\Gamma$ may be identified with a finite subgroup of aut $(G)$, still denoted by $\Gamma$. By Proposition 2.3 , $G$ has a $\Gamma$-stable maximal compact subgroup $K$. Its Lie algebra $\mathfrak{k}_{0}$ is also $\Gamma$-stable.

The Killing form of $\mathfrak{g}_{0}$ is nondegenerate. Let $\mathfrak{p}_{0}$ be the orthogonal complement of $\mathfrak{k}_{0}$ with respect to the Killing form. Then $\mathfrak{g}_{0}=\mathfrak{k}_{0}+\mathfrak{p}_{0}$ is a Cartan decomposition. Let $\theta$ be the corresponding Cartan involution. Each element of $\Gamma$ preserves $\mathfrak{k}_{0}$ and the Killing form, so it also preserves $\mathfrak{p}_{0}$ and therefore commutes with $\theta$.

The second statement of Corollary 2.4(a), on the uniqueness of $\theta$, is given by the proof of [4, Chap. III, Thms. 7.1, 7.2]. We briefly provide its outline here. Suppose that for $j=1,2, \mathfrak{g}_{0}=\mathfrak{k}_{j}+\mathfrak{p}_{j}$ are Cartan decompositions which are stable under every element of $\Gamma$. Then $\mathfrak{u}_{j}=\mathfrak{k}_{j}+i \mathfrak{p}_{j}$ are compact real forms of $\mathfrak{g}$. Let $\tau_{j}$ be the conjugation of $\mathfrak{g}$ with respect to $\mathfrak{u}_{j}$. Then $\left(\tau_{1} \tau_{2}\right)^{2}$ is a positive definite semisimple inner $\mathfrak{g}$-automorphism which preserves $\mathfrak{g}_{0}$, and $\left(\tau_{1} \tau_{2}\right)^{\frac{1}{2}}$ maps $\mathfrak{k}_{2}$ to $\mathfrak{k}_{1}$ and maps $\mathfrak{p}_{2}$ to $\mathfrak{p}_{1}$. Since $\tau_{1}$ and $\tau_{2}$ centralize $\Gamma$, so does $\left(\tau_{1} \tau_{2}\right)^{\frac{1}{2}}$. Hence $\left(\tau_{1} \tau_{2}\right)^{\frac{1}{2}}$ is the desired automorphism. This proves Corollary 2.4(a).

For Corollary 2.4(b), let $\theta$ and $\sigma$ be commuting $\mathfrak{g}$-automorphisms, where $\operatorname{ord}(\theta)=$ 2 and $\operatorname{ord}(\sigma)<\infty$. Write $\mathfrak{g}=\mathfrak{k}+\mathfrak{p}$, where $\mathfrak{k}$ and $\mathfrak{p}$ are the \pm 1 -eigenspaces of $\theta$. Treat $\mathfrak{g}$ as a real simple Lie algebra and apply Corollary 2.4 (a) to $\Gamma=\langle\theta, \sigma\rangle \subset \operatorname{aut}(\mathfrak{g})$; there exists a Cartan decomposition of $\mathfrak{g}$ which is stable under $\theta$ and $\sigma$. Since $\mathfrak{g}$ is complex, this Cartan decomposition is given by $\mathfrak{g}=\mathfrak{u}_{0}+i \mathfrak{u}_{0}$ for some compact real form $\mathfrak{u}_{0}$ of $\mathfrak{g}$. Here $\theta$ and $\sigma$ preserve $\mathfrak{u}_{0}$ and $i \mathfrak{u}_{0}$. Let

$$
\mathfrak{k}_{0}=\mathfrak{u}_{0} \cap \mathfrak{k}, \mathfrak{p}_{0}=i \mathfrak{u}_{0} \cap \mathfrak{p}, \mathfrak{g}_{0}=\mathfrak{k}_{0}+\mathfrak{p}_{0} .
$$


We claim that $\mathfrak{g}_{0}$ is the desired real form. By (2.4) and properties of $\mathfrak{k}, \mathfrak{p}$ and $\mathfrak{u}_{0}$,

$$
\left[\mathfrak{k}_{0}, \mathfrak{k}_{0}\right] \subset \mathfrak{k}_{0},\left[\mathfrak{k}_{0}, \mathfrak{p}_{0}\right] \subset \mathfrak{p}_{0} \quad, \quad\left[\mathfrak{p}_{0}, \mathfrak{p}_{0}\right] \subset \mathfrak{k}_{0} .
$$

Hence $\mathfrak{g}_{0}$ is a real Lie subalgebra of $\mathfrak{g}$. Also,

$$
\begin{array}{rlrl}
\operatorname{dim}_{\mathbb{C}} \mathfrak{g} & =\operatorname{dim}_{\mathbb{R}} \mathfrak{u}_{0} & & \\
& =\operatorname{dim}_{\mathbb{R}}\left(\mathfrak{u}_{0} \cap \mathfrak{k}\right)+\operatorname{dim}_{\mathbb{R}}\left(\mathfrak{u}_{0} \cap \mathfrak{p}\right) & & \text { as } \mathfrak{u}_{0} \text { is } \theta \text {-stable } \\
& =\operatorname{dim}_{\mathbb{R}}\left(\mathfrak{u}_{0} \cap \mathfrak{k}\right)+\operatorname{dim}_{\mathbb{R}}\left(i \mathfrak{u}_{0} \cap \mathfrak{p}\right) & & \text { as } \mathfrak{p}=i \mathfrak{p} \\
& =\operatorname{dim}_{\mathbb{R}} \mathfrak{k}_{0}+\operatorname{dim}_{\mathbb{R}} \mathfrak{p}_{0} & & \text { by (2.4) } \\
& =\operatorname{dim}_{\mathbb{R}} \mathfrak{g}_{0} . &
\end{array}
$$

Furthermore,

$$
\begin{aligned}
& \mathfrak{g}_{0}+i \mathfrak{g}_{0}=\mathfrak{k}_{0}+\mathfrak{p}_{0}+i \mathfrak{k}_{0}+i \mathfrak{p}_{0} \\
& =\left(\mathfrak{u}_{0} \cap \mathfrak{k}\right)+\left(i \mathfrak{u}_{0} \cap \mathfrak{p}\right)+\left(i \mathfrak{u}_{0} \cap i \mathfrak{k}\right)+\left(\mathfrak{u}_{0} \cap i \mathfrak{p}\right) \quad \text { by (2.4) } \\
& =\left(\mathfrak{u}_{0} \cap \mathfrak{k}\right)+\left(i \mathfrak{u}_{0} \cap \mathfrak{p}\right)+\left(i \mathfrak{u}_{0} \cap \mathfrak{k}\right)+\left(\mathfrak{u}_{0} \cap \mathfrak{p}\right) \quad \text { as } \mathfrak{k}=i \mathfrak{k} \text { and } \mathfrak{p}=i \mathfrak{p} \\
& =\mathfrak{u}_{0}+i \mathfrak{u}_{0} \\
& =\mathfrak{g} \text {. } \\
& \text { as } \mathfrak{u}_{0} \text { is } \theta \text {-stable }
\end{aligned}
$$

By (2.6) and (2.7), $\mathfrak{g}_{0}$ is a real form of $\mathfrak{g}$.

Since $\mathfrak{k}_{0} \subset \mathfrak{u}_{0}$ and $\mathfrak{p}_{0} \subset i \mathfrak{u}_{0}$, the Killing form is negative definite on $\mathfrak{k}_{0}$ and positive definite on $\mathfrak{p}_{0}$. So together with (2.5), it follows that $\mathfrak{g}_{0}=\mathfrak{k}_{0}+\mathfrak{p}_{0}$ is a Cartan decomposition, with Cartan involution $\theta$.

Since $\sigma$ preserves $\mathfrak{u}_{0}, i \mathfrak{u}_{0}$ and commutes with $\theta$, it also preserves $\mathfrak{k}$ and $\mathfrak{p}$, as well as $\mathfrak{k}_{0}=\mathfrak{u}_{0} \cap \mathfrak{k}$ and $\mathfrak{p}_{0}=i \mathfrak{u}_{0} \cap \mathfrak{p}$. Hence $\mathfrak{g}_{0}=\mathfrak{k}_{0}+\mathfrak{p}_{0}$ is $\sigma$-stable. This proves Corollary 2.4(b).

Let $\sigma$ be a finite order $\mathfrak{g}_{0}$-automorphism. Apply Corollary 2.4(a) to $\Gamma=\langle\sigma\rangle \subset$ $\operatorname{aut}\left(\mathfrak{g}_{0}\right)$. We identify $\sigma$ with a pair of commuting automorphisms $(\theta, \sigma)$ on $\mathfrak{g}$, where $\theta$ is the Cartan involution on $\mathfrak{g}_{0}$. The pair $(\theta, \sigma)$ is also related to involution on an affine Kac-Moody Lie algebra [9]: We can use $\sigma$ to construct an affine Kac-Moody Lie algebra [7; then $\theta$ leads to an involution on the algebra.

Proposition 2.5. Let $\sigma$ be a finite order $\mathfrak{k}$-automorphism. Then $\mathfrak{k}$ has a $\sigma$-stable Cartan subalgebra $\mathfrak{h}$ which has a $\sigma$-stable simple system. Furthermore, $\mathfrak{h}$ is unique up to conjugation by an inner $\mathfrak{k}$-automorphism which commutes with $\sigma$.

Proof. The existence of a $\sigma$-stable Cartan subalgebra which has a $\sigma$-stable simple system is proved in 3 and [11, $§ 4.4 .2]$. We now prove its uniqueness. Suppose that $\mathfrak{h}$ and $\mathfrak{h}_{1}$ are such Cartan subalgebras. Let $\Pi$ and $\Pi_{1}$ be their $\sigma$-stable simple systems, respectively. Let $K^{\mathbb{C}}$ be the connected and simply connected complex Lie group whose Lie algebra is $\mathfrak{k}$. Then $\sigma$ lifts to an automorphism on $K^{\mathbb{C}}$, still denoted by $\sigma$. Let $H$ be the Cartan subgroup of $K^{\mathbb{C}}$ whose Lie algebra is $\mathfrak{h}$, and let $U^{ \pm}$be the maximal nilpotent subgroup whose Lie algebra $\mathfrak{u}^{ \pm}$contains the root spaces of $\pm \Pi$. We define $\mathfrak{u}_{1}^{+}$similarly from $\Pi_{1}$.

Let $K^{\mathbb{C}}$ act on $\mathfrak{k}$ by the adjoint representation. By the conjugacy theorems of Cartan and Borel subalgebras of $\mathfrak{k}$ [5. Ch. 16], there exists $g_{1} \in K^{\mathbb{C}}$ such that $g_{1} \cdot \mathfrak{h}=\mathfrak{h}_{1}$ and $g_{1} \cdot \mathfrak{u}^{+}=\mathfrak{u}_{1}^{+}$. The adjoint action of $\sigma\left(g_{1}\right)$ also has the same property because $\mathfrak{h}, \mathfrak{h}_{1}, \Pi, \Pi_{1}$ are all $\sigma$-stable. Therefore, since $H$ is the stabilizer of $\mathfrak{h}$ and $\mathfrak{u}^{+}$in $K^{\mathbb{C}}$, there exists $h \in H$ such that

$$
\sigma\left(g_{1}\right)=g_{1} h .
$$


Let $N$ be the normalizer of $H$ in $K^{\mathbb{C}}$. By the Bruhat decomposition (see for example [12, §8.3]), there exist unique $u \in U^{+}, n \in N$ and $u^{\prime} \in U^{+} \cap n^{-1} U^{-} n$ such that $g_{1}=u n u^{\prime}$. By (2.8),

$$
\sigma(u) \sigma(n) \sigma\left(u^{\prime}\right)=\sigma\left(u n u^{\prime}\right)=\sigma\left(g_{1}\right)=g_{1} h=u n u^{\prime} h=u(n h)\left(h^{-1} u^{\prime} h\right) .
$$

Since $\mathfrak{h}$ and $\Pi$ are $\sigma$-stable, so are $U^{ \pm}$and $N$. Therefore, (2.9) implies that

$$
\sigma(u)=u, \quad \sigma(n)=n h, \quad \sigma\left(u^{\prime}\right)=h^{-1} u^{\prime} h .
$$

Let $g=g_{1} n^{-1}$. We claim that the adjoint action of $g$ commutes with $\sigma$ and maps $\mathfrak{h}$ to $\mathfrak{h}_{1}$. By (2.8) and (2.10), $\sigma(g)=\sigma\left(g_{1}\right) \sigma(n)^{-1}=\left(g_{1} h\right)\left(h^{-1} n^{-1}\right)=g$. Therefore, for all $x \in K^{\mathbb{C}}$,

$$
\sigma\left(g x g^{-1}\right)=\sigma(g) \sigma(x) \sigma(g)^{-1}=g \sigma(x) g^{-1} .
$$

We let $\sigma$ denote both a Lie group automorphism and a Lie algebra automorphism. Taking the differential of (2.11), we see that $\sigma(g \cdot X)=g \cdot(\sigma X)$ for all $X \in \mathfrak{k}$, namely the adjoint action of $g$ commutes with $\sigma$.

Since $n$ normalizes $H$, we have $g \cdot \mathfrak{h}=g_{1} n^{-1} \cdot \mathfrak{h}=g_{1} \cdot \mathfrak{h}=\mathfrak{h}_{1}$. So the adjoint action of $g$ maps $\mathfrak{h}$ to $\mathfrak{h}_{1}$. This proves the proposition.

Proposition 2.6. Suppose that $\sigma \in \operatorname{aut}(\mathfrak{g})$ preserves a Cartan subalgebra of $\mathfrak{g}$ and acts as multiplication by $z, v \in \mathbb{C}$ on the root spaces $\mathfrak{g}_{\alpha}, \mathfrak{g}_{\beta}$, respectively. Then:

(a) $\sigma$ acts as multiplication by $z v$ on $\mathfrak{g}_{\alpha+\beta}$;

(b) $\sigma$ acts as multiplication by $z^{-1}$ on $\mathfrak{g}_{-\alpha}$.

Proof. For part (a), if $X_{\alpha} \in \mathfrak{g}_{\alpha}$ and $X_{\beta} \in \mathfrak{g}_{\beta}$, then

$$
\sigma\left[X_{\alpha}, X_{\beta}\right]=\left[\sigma X_{\alpha}, \sigma X_{\beta}\right]=\left[z X_{\alpha}, v X_{\beta}\right]=z v\left[X_{\alpha}, X_{\beta}\right]
$$

Next we prove part (b). We first consider the special case $\mathfrak{g}=\mathfrak{s l}(2, \mathbb{C})$. Let $H, E, F$ be its standard triple, where $H$ is diagonal, $E$ is upper triangular and $F$ is lower triangular. Suppose that $\sigma E=z E$. If $\sigma H=u H$, then

$$
2 z E=2 \sigma E=\sigma[H, E]=[\sigma H, \sigma E]=u z[H, E]=2 u z E .
$$

It forces $u=1$. Consequently, if $\sigma F=x F$, then

$$
H=\sigma H=\sigma[E, F]=[\sigma E, \sigma F]=z x[E, F]=z x H .
$$

It forces $x=z^{-1}$.

For general $\mathfrak{g}$, suppose that $\sigma$ acts by $z$ on $\mathfrak{g}_{\alpha}$. There exists some $E \in \mathfrak{g}_{\alpha}$, $F \in \mathfrak{g}_{-\alpha}$ and semisimple element $H$ such that $H, E, F$ form an $\mathfrak{s l}(2, \mathbb{C})$-triple in $\mathfrak{g}$. By the above argument, it follows that $\sigma$ acts by $z^{-1}$ on $\mathfrak{g}_{-\alpha}$. This proves part (b).

Suppose that the hypothesis of Proposition 2.6 is modified to let $\sigma$ preserve a Cartan subalgebra of $\mathfrak{k}$ instead of $\mathfrak{g}$. If $\mathfrak{g}$ is of equal rank type (1.6) (a), (b), the same conclusions follow. If $\mathfrak{g}$ is of nonequal rank type (1.6) (c), then we need to deal with $\mathfrak{g}_{\alpha} \cap \mathfrak{k}$ or $\mathfrak{g}_{\alpha} \cap \mathfrak{p}$, and the conclusions are expressed as $\mathfrak{g}_{\alpha+\beta} \cap \mathfrak{k}$ or $\mathfrak{g}_{\alpha+\beta} \cap \mathfrak{p}$, and so on.

Finally we recall the reflection of roots. Given a root $\alpha$, let $r_{\alpha}$ denote the reflection which sends $\alpha$ to $-\alpha$ and fixes the hyperplane perpendicular to $\alpha$. Let $\alpha--\beta$ denote "vertices $\alpha$ and $\beta$ are joined by a single edge", let $\alpha==>\beta$ denote "vertices $\alpha$ and $\beta$ are joined by a double edge with arrow pointing toward $\beta$ ", and so on. 
Proposition 2.7. Let $\alpha$ and $\beta$ be adjacent vertices of a Dynkin diagram.

(a) If $\alpha--\beta$, then $r_{\alpha} \beta=\alpha+\beta$.

(b) If $\alpha==>\beta$, then $r_{\alpha} \beta=\alpha+\beta$.

(c) If $\alpha<==\beta$, then $r_{\alpha} \beta=2 \alpha+\beta$.

(d) If $\alpha \equiv \equiv>\beta$, then $r_{\alpha} \beta=\alpha+\beta$.

(e) If $\alpha<\equiv \equiv \beta$, then $r_{\alpha} \beta=3 \alpha+\beta$.

By drawing the picture of the rank-two root system generated by $\alpha$ and $\beta$, we immediately obtain the above proposition. It can also be computed easily, for example Proposition 2.7(a) is $r_{\alpha} \beta=\beta-2 \frac{(\beta, \alpha)}{(\alpha, \alpha)} \alpha=\beta+\alpha$, and so on.

\section{PAinted Diagrams AND REAL Forms}

Corollary 2.2 and (2.2) imply that the painted diagrams correspond to the real simple Lie algebras. We list all of them in Figures 1,2 and 3 to facilitate our references later, though they also appear in [11, Table 7]. For exceptional Lie algebras, we use Cartan's notation $\mathfrak{g}_{n(m)}$ (see for instance [4, Ch. X-6]) to denote the real form of $\mathfrak{g}_{n}$ with $m=\operatorname{dim} \mathfrak{p}-\operatorname{dim} \mathfrak{k}$.

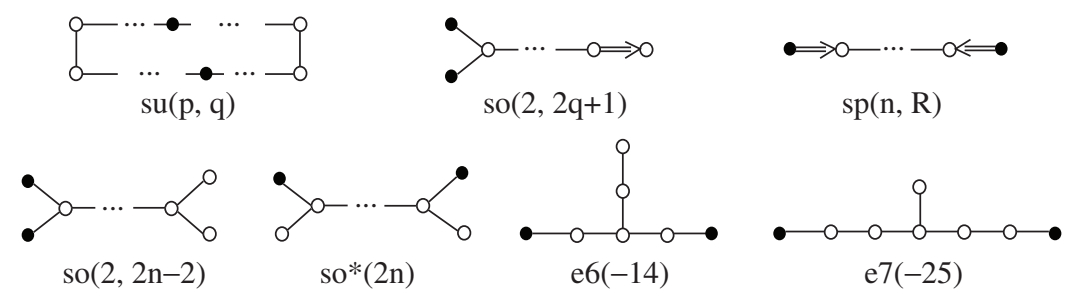

FiguRE 1. Hermitian type

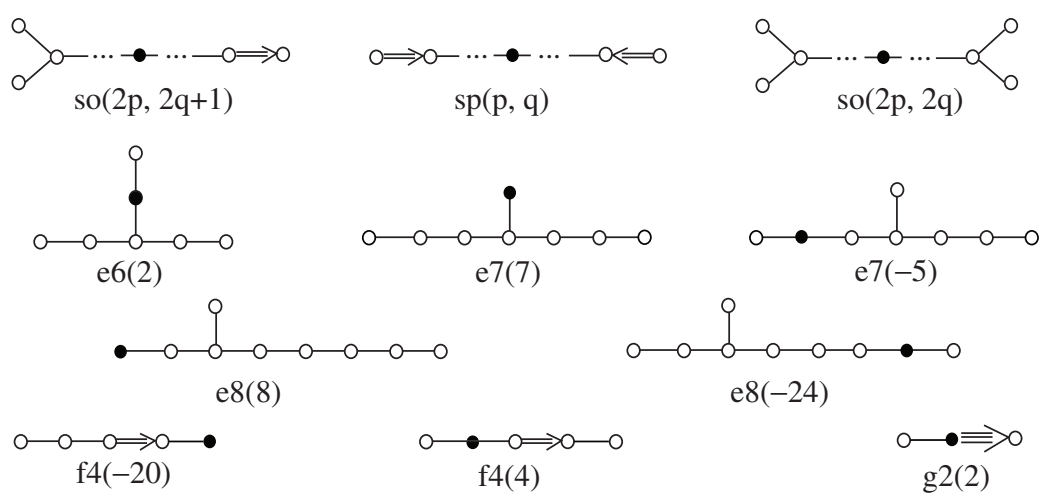

Figure 2. Non-Hermitian equal rank type

Figures 1, 2 and 3 list all the real forms of complex simple Lie algebras up to isomorphism, along with their painted diagrams. Two isomorphic real forms $\mathfrak{g}_{0}, \mathfrak{g}_{1} \subset \mathfrak{g}$ are said to be inner isomorphic if there exists an isomorphism $\mathfrak{g}_{0} \longrightarrow \mathfrak{g}_{1}$ which extends to $\operatorname{int}(\mathfrak{g})$. Figure 4 shows the painted diagrams of all isomorphic real forms that are not inner isomorphic, so that Figures 1, 2, 3 and 4 provide a 

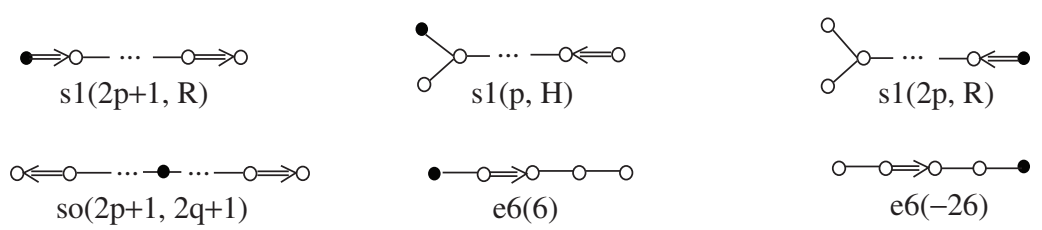

FIGURE 3. Non-Hermitian nonequal rank type
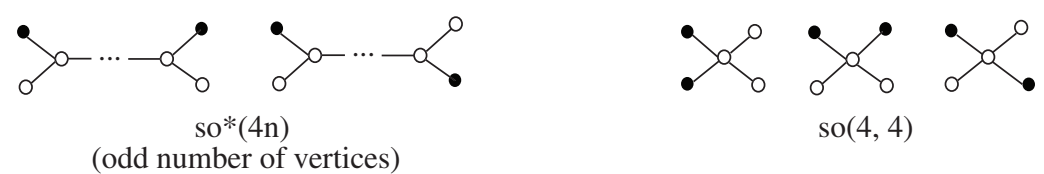

FigURE 4. Isomorphic real forms that are not inner isomorphic

complete list of all real forms up to inner isomorphism. Its proof involves technical computations, so we leave it as an appendix in Section 10.

\section{Diagram markings And aUtomorphisms}

In this section, we prove Theorem 1.3. A marking on a diagram $\mathcal{D}$ is a pair $(c, d)$,

$$
c: \mathcal{D} \longrightarrow U, d \in \operatorname{aut}(\mathcal{D})
$$

where $U$ is the multiplicative group (1.3). The diagram $\mathcal{D}$ can be $\mathrm{D}_{\mathfrak{k}}, \mathrm{D}, \mathrm{D}^{r}$ or $\mathrm{P}^{r}$. If $\mathcal{D}=\mathrm{P}^{r}$, then aut $\left(\mathrm{P}^{r}\right)$ is also understood to preserve vertex colors. The idea is to show that suitable markings represent finite order automorphisms on the related Lie algebras, in the sense of Definition [1.2, For instance Theorem 2.1 uses certain markings on $\mathrm{D}^{r}$ with $d=1$, and it classifies aut $m(\mathfrak{g})$.

The Vogan diagrams $\left[8\right.$ are the markings on D which satisfy $c_{\alpha}= \pm 1, \operatorname{ord}(d)=$ 1,2 , and also $c_{\alpha}=1$ whenever $d \alpha \neq \alpha$.

Theorem 4.1 ([8]). Every Vogan diagram represents a unique real form, or equivalently an involution, on $\mathfrak{g}$. Conversely, every real form or involution on $\mathfrak{g}$ is represented by a Vogan diagram.

The following extends Theorem 4.1 to higher order $\mathfrak{g}$-automorphisms.

Theorem 4.2. Every marking on $\mathrm{D}$ represents a unique finite order $\mathfrak{g}$-automorphism. Conversely, every finite order $\mathfrak{g}$-automorphism is represented by a marking on $\mathrm{D}$.

Proof. Let $(c, d)$ be a marking on D. Suppose that $d=1$. By Proposition 2.6. it represents a unique $\mathfrak{g}$-automorphism which acts as an identity on the Cartan subalgebra and acts as multiplication by $\Pi_{\mathrm{D}} c_{\alpha}^{s_{\alpha}}$ on the root space of $\sum_{\mathrm{D}} s_{\alpha} \alpha$.

Next we consider $d \neq 1$. Write $(c, d)$ as the composite of two other markings,

$$
(c, d)=(c, 1) \cdot(1, d) .
$$

Here $(c, 1) \cdot(1, d)$ indicates the marking $(1, d)$ followed by $(c, 1)$, where $(c, 1)$ has trivial diagram automorphism and $(1, d)$ assigns $1 \in U$ to all the vertices. By Definition 1.2. if $(1, d)$ and $(c, 1)$ represent $\mathfrak{g}$-automorphisms $\sigma_{1}$ and $\sigma_{2}$, respectively, then $(c, 1) \cdot(1, d)$ represents $\sigma_{2} \cdot \sigma_{1}$. So it suffices to consider $(1, d)$ and $(c, 1)$ separately. 
If $\operatorname{ord}(d)=2$, Theorem 4.1 says that the Vogan diagram $(1, d)$ represents some $\sigma_{1} \in \operatorname{aut}_{2}(\mathfrak{g})$. Also, by the above argument, $(c, 1)$ represents some $\sigma_{2} \in \operatorname{aut}_{m}(\mathfrak{g})$. Consequently $(c, d)$ represents $\sigma_{2} \cdot \sigma_{1} \in \operatorname{aut}(\mathfrak{g})$. The only case with $\operatorname{ord}(d)>2$ happens on $D_{4}$. Here $d$ is the composite of two diagram involutions $d_{1}, d_{2}$, so that $(c, d)=(c, 1) \cdot\left(1, d_{2}\right) \cdot\left(1, d_{1}\right)$. By the above arguments, each of $(c, 1),\left(1, d_{2}\right),\left(1, d_{1}\right)$ represents a finite order $\mathfrak{g}$-automorphism, so $(c, d)$ represents their composite. This proves the first statement of the theorem.

Conversely, let $\sigma$ be a finite order $\mathfrak{g}$-automorphism. By Proposition 2.5, there exists a $\sigma$-stable Cartan subalgebra which has a $\sigma$-stable simple system. Let D be its corresponding Dynkin diagram. Then $\sigma$ induces $d \in \operatorname{aut}(\mathrm{D})$ by $\sigma \mathfrak{g}_{\alpha}=\mathfrak{g}_{d \alpha}$. By Theorem 4.1, $d$ represents a finite order $\mathfrak{g}$-automorphism $\sigma_{d}$ (if $\mathfrak{g}=D_{4}$ and $\operatorname{ord}(d)=3$, we may write $d$ as the composite of two involutions and apply Theorem 4.1 to both involutions). There are canonical root vectors $\left\{X_{\alpha}\right\}_{\mathrm{D}}$ of $\mathfrak{g}_{\alpha}$ such that $\sigma_{d} X_{\alpha}=X_{d \alpha}$ [8, Thm. 6.88]. Since $\sigma X_{\alpha}, X_{d \alpha} \in \mathfrak{g}_{d \alpha}$, there exist $\left\{c_{\alpha}\right\}_{\mathrm{D}} \subset \mathbb{C}$ such that $\sigma X_{\alpha}=c_{d \alpha} X_{d \alpha}$.

Finally, we show that by renormalizing $\left\{X_{\alpha}\right\}_{\mathrm{D}}$, we can make $c_{\alpha} \in U$ for all $\alpha \in$ D. If $d \gamma=\gamma$, then $c_{\gamma}$ is an eigenvalue of $\sigma$, and it follows from $\operatorname{ord}(\sigma)<\infty$ that $c_{\gamma} \in U$. It remains to consider $d \gamma \neq \gamma$. Fix $\gamma \in \mathcal{O}$, where $\mathcal{O}$ is a $d$-orbit and $|\mathcal{O}|=k>1$. By Definition 1.2 ,

$$
\sigma\left(X_{\gamma}\right)=\sigma_{c} \sigma_{d}\left(X_{\gamma}\right)=\sigma_{c}\left(X_{d \gamma}\right)=c_{d \gamma} X_{d \gamma} .
$$

We compute inductively and get $\sigma^{k}\left(X_{\gamma}\right)=c_{d^{k} \gamma} c_{d^{k-1} \gamma} \ldots c_{d \gamma} X_{\gamma}$. Let $\operatorname{ord}\left(\sigma, X_{\gamma}\right)$ be the order of $X_{\gamma}$ under the action of $\sigma$. Then

$$
\operatorname{ord}\left(\sigma, X_{\gamma}\right)=k \operatorname{ord}\left(c_{d^{k} \gamma} c_{d^{k-1} \gamma} \ldots c_{d \gamma}\right)=|\mathcal{O}| \operatorname{ord}\left(\Pi_{\alpha \in \mathcal{O}} c_{\alpha}\right) .
$$

Since $\sigma$ is of finite order, (4.2) implies that $\operatorname{ord}\left(\Pi_{\alpha \in \mathcal{O}} c_{\alpha}\right)<\infty$. There are many ways to replace $\left\{c_{\alpha}\right\}_{\mathcal{O}}$ by $\left\{c_{\alpha}^{\prime}\right\}_{\mathcal{O}} \subset U$. For example, if we replace $\left\{X_{\alpha}\right\}_{\mathcal{O}}$ by $\left\{\sigma^{i} X_{\gamma}\right\}_{i=1}^{k}$, then $\left\{c_{\alpha}^{\prime}\right\}_{\mathcal{O}}=\left\{1,1, \ldots, 1, \Pi_{\alpha \in \mathcal{O}} c_{\alpha}\right\} \subset U$. This proves the theorem.

We have obtained two ways to represent a finite order $\mathfrak{g}$-automorphism $\sigma$ :

(a) a marking $c$ on $\mathrm{D}^{r}$ in Theorem 2.1

(b) a marking $(c, d)$ on $\mathrm{D}$ in Theorem 4.2 ,

They reveal whether $\sigma$ is an inner or outer automorphism by [4, Ch. X-5, Thm. 5.16] and [5, §16.5]:

(a) $\sigma \in \operatorname{int}(\mathfrak{g}) \Longleftrightarrow r=1$ in (4.3)(a) $\Longleftrightarrow d=1$ in (4.3)(b);

(b) $\sigma \in \operatorname{out}(\mathfrak{g}) \Longleftrightarrow r=2,3$ in (4.3)(a) $\Longleftrightarrow d \neq 1$ in (4.3)(b).

Theorem 2.1 has imposed conditions so that the marking is unique. But the marking in Theorem 4.2 is not unique, so it remains to determine those markings that represent conjugate $\mathfrak{g}$-automorphisms. This has been done for Vogan diagrams for $\mathfrak{g}$-involutions [1]. For higher order automorphisms, since we intend to apply Theorem 4.2 to $D_{\mathfrak{k}}$ and $\operatorname{aut}_{m}([\mathfrak{k}, \mathfrak{k}])$, we will deal with this issue on the entire $\mathfrak{g}$ in Section 5. Recall that $\mathrm{D}_{\mathfrak{k}}$ is the subdiagram (1.2) of white vertices of $\mathrm{P}^{r}$.

Corollary 4.3. Every marking on $\mathrm{D}_{\mathfrak{k}}$ represents a unique finite order automorphism on $[\mathfrak{k}, \mathfrak{k}]$. Conversely, every finite order automorphism on $[\mathfrak{k}, \mathfrak{k}]$ is represented by a marking on $\mathrm{D}_{\mathfrak{k}}$.

Proof. The Dynkin diagram of $[\mathfrak{k}, \mathfrak{k}]$ is $\mathrm{D}_{\mathfrak{k}}$. Since Theorem 4.2 also holds for semisimple Lie algebras, we may apply it to $\mathrm{D}_{\mathfrak{k}}$ and obtain this corollary. 
Let $n$ and $m$ be positive integers. The following proposition gives a necessary condition for the extension of $\sigma \in \operatorname{aut}_{n}(\mathfrak{k})$ to $\operatorname{aut}_{m}(\mathfrak{g})$.

Proposition 4.4. Let $\sigma \in$ aut $_{n}(\mathfrak{k})$ be represented by a marking $(c, d)$ on $\mathrm{D}_{\mathfrak{k}}$. If $\sigma$ extends to some $\operatorname{aut}_{m}(\mathfrak{g})$, then d extends to aut $\left(\mathrm{P}^{r}\right)$.

Proof. Suppose that $\sigma$ extends to $\operatorname{aut}_{m}(\mathfrak{g})$. Then it permutes the extreme weight spaces of $\mathfrak{p}$. The simple roots of $\mathfrak{k}$ correspond to $D_{\mathfrak{k}}$. Since the restriction of $\sigma$ to $\mathfrak{k}$ is represented by a marking on $D_{\mathfrak{k}}$, it follows that $\sigma$ permutes the simple root spaces of $\mathfrak{k}$. Therefore, $\sigma$ indeed permutes the corresponding lowest weight spaces of $\mathfrak{p}$. These lowest weights are the black vertices of $\mathrm{P}^{r}$, so $d$ extends to aut $\left(\mathrm{P}^{r}\right)$.

In (2.2) (a), $\mathfrak{k}$ has nontrivial center $\mathfrak{z}$, so $D_{\mathfrak{k}}$ represents $[\mathfrak{k}, \mathfrak{k}]$ rather than $\mathfrak{k}$. However, the following proposition says that if $\sigma \in \operatorname{aut}\left(\mathfrak{g}_{0}\right)$ is represented by a marking on $\mathrm{P}^{r}$, then its value on $\mathfrak{z}$ is determined.

Proposition 4.5. Suppose that a marking $(c, d)$ on $\mathrm{P}^{r}$ represents $\sigma \in \operatorname{aut}\left(\mathfrak{g}_{0}\right)$.

(a) If $d$ fixes the black vertices, then $\sigma=1$ on $\mathfrak{z}$.

(b) If $d$ interchanges the two black vertices, then $\sigma=-1$ on $\mathfrak{z}$.

Proof. By (2.2), we may assume that $\mathfrak{g}_{0}$ is of Hermitian type, for otherwise $\mathfrak{z}=0$ and there is nothing to prove. Its painted diagram $\mathrm{P}^{1}$ has two black vertices $\{\gamma, \delta\}$ with $a_{\gamma}=a_{\delta}=1$, and they are the only vertices of $\mathrm{P}^{1}$ which do not annihilate $\mathfrak{z}$. Let $B$ be the Killing form of $\mathfrak{g}$. Let $X_{\alpha} \in \mathfrak{g}_{\alpha}$ be root vectors such that

$$
B\left(\left[X_{\alpha}, X_{-\alpha}\right], \cdot\right)=\alpha .
$$

We first prove part (a), where $d$ fixes $\gamma$ and $\delta$. By Proposition 2.6(b),

$$
\sigma\left[X_{\gamma}, X_{-\gamma}\right]=\left[\sigma X_{\gamma}, \sigma X_{-\gamma}\right]=\left[c_{\gamma} X_{\gamma}, c_{\gamma}^{-1} X_{-\gamma}\right]=\left[X_{\gamma}, X_{-\gamma}\right] .
$$

Pick $0 \neq Z \in \mathfrak{z}$. Since $\sigma$ preserves the Killing form, by (4.5) and (4.6),

$$
\gamma(Z)=B\left(Z,\left[X_{\gamma}, X_{-\gamma}\right]\right)=B\left(\sigma Z,\left[\sigma X_{\gamma}, \sigma X_{-\gamma}\right]\right)=B\left(\sigma Z,\left[X_{\gamma}, X_{-\gamma}\right]\right)=\gamma(\sigma Z) .
$$

Since $\gamma$ does not annihilate $Z$, it implies that $\sigma=1$ on $\mathfrak{z}$. This proves part (a).

Next we prove part (b), where $d$ interchanges $\gamma$ and $\delta$. There are root vectors $X_{ \pm \gamma} \in \mathfrak{g}_{ \pm \gamma}$ and $X_{ \pm \delta} \in \mathfrak{g}_{ \pm \delta}$ such that $\sigma X_{\gamma}=X_{\delta}$ and $\sigma X_{-\gamma}=X_{-\delta}$, Ch. VI-8, (6.91)]. Pick $0 \neq Z \in \mathfrak{z}$. By (4.5),

$$
\gamma(Z)=B\left(Z,\left[X_{\gamma}, X_{-\gamma}\right]\right)=B\left(\sigma Z,\left[\sigma X_{\gamma}, \sigma X_{-\gamma}\right]\right)=B\left(\sigma Z,\left[X_{\delta}, X_{-\delta}\right]\right)=\delta(\sigma Z) .
$$

Since $\gamma$ and $\delta$ are the only vertices of $\mathrm{P}^{1}$ which do not annihilate $Z$, by (1.1) we get $\gamma(Z)+\delta(Z)=0$. Then (4.7) implies that $\sigma Z=-Z$. This proves part (b).

In [2], we prove the special case of Theorem 1.3 for $\mathfrak{g}_{0}$-involutions. We include it here for future reference. Recall that the admissible markings are defined in (1.5).

Theorem 4.6 ([2, Thm.1.3]). An admissible marking $(c, d)$ on $\mathrm{P}^{r}$ such that

$$
c: \mathrm{P}^{r} \longrightarrow\{ \pm 1\}, c_{\alpha}=1 \text { whenever } d \alpha \neq \alpha, \operatorname{ord}(d) \in\{1,2\}
$$

represents a unique $\mathfrak{g}_{0}$-involution. Conversely, every $\mathfrak{g}_{0}$-involution is represented by an admissible marking on $\mathrm{P}^{r}$ which satisfies (4.8).

Theorem 4.7. A marking on $\mathrm{P}^{r}$ represents a finite order $\mathfrak{g}_{0}$-automorphism if and only if it is admissible. 
Proof. Let $(c, d)$ be a marking on $\mathrm{P}^{r}$. Consider the following cases:

$$
\begin{aligned}
& \text { (a) } d=1, \\
& \text { (b) } \operatorname{ord}(d)=2, \\
& \text { (c) } \operatorname{ord}(d)>2 .
\end{aligned}
$$

We start with (4.9) (a), where $d=1$. By Proposition 2.6 and Corollary 4.3 the restriction of $c$ to $D_{\mathfrak{k}}$ represents a $\mathfrak{k}$-automorphism $\sigma_{\mathfrak{k}}$ by

$$
\sigma_{\mathfrak{k}} X=\Pi_{\mathrm{D}_{\mathfrak{k}}} c_{\alpha}^{q_{\alpha}} X, X \in \mathfrak{k}_{\sum_{\mathrm{D}_{\mathfrak{k}}} q_{\alpha} \alpha},
$$

where $\mathfrak{k}_{\sum_{\mathrm{D}_{\mathfrak{k}}} q_{\alpha} \alpha}$ is the root space of $\sum_{\mathrm{D}_{\mathfrak{k}}} q_{\alpha} \alpha$. Let $\gamma, \delta$ be the black vertices of $\mathrm{P}^{r}$ (if $\mathrm{P}^{r}$ has only one black vertex, then $\gamma=\delta$ ). Since $\gamma, \delta$ are the lowest weights of $\mathfrak{p}$, the weights of $\mathfrak{p}$ are of the form $\gamma+\sum_{D_{\mathfrak{k}}} p_{\alpha} \alpha$ or $\delta+\sum_{D_{\mathfrak{k}}} p_{\alpha} \alpha$. Define a vector space automorphism $\sigma_{\mathfrak{p}}$ on the weight spaces of $\mathfrak{p}$ by

$$
\sigma_{\mathfrak{p}} X=c_{\gamma} \Pi_{D_{\mathfrak{k}}} c_{\alpha}^{p_{\alpha}} X, X \in \mathfrak{p}_{\gamma+\sum_{\mathrm{D}_{\mathfrak{k}}} p_{\alpha} \alpha},
$$

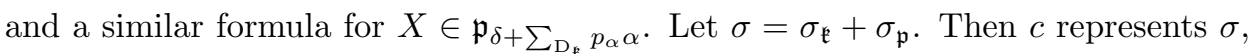
but we need to check whether $\sigma$ is a $\mathfrak{g}$-automorphism, namely $\sigma[X, Y]=[\sigma X, \sigma Y]$. By (4.10), it holds for $X, Y \in \mathfrak{k}$. By (4.11), it holds for $X \in \mathfrak{k}$ and $Y \in \mathfrak{p}$. So it remains to consider $X, Y \in \mathfrak{p}$. It suffices to let $[X, Y] \in \mathfrak{k}_{\beta} \subset \mathfrak{k}$. Suppose that $\beta=\sum_{\mathrm{D}_{\mathrm{k}}} q_{\alpha} \alpha=\gamma+\delta+\sum_{\mathrm{D}_{\mathrm{e}}} p_{\alpha} \alpha$ (if $\mathrm{P}^{r}$ has only one black vertex $\gamma$, then $\gamma+\delta=2 \gamma$ ). Then (4.10) and (4.11) imply that

$$
\sigma_{\mathfrak{k}}[X, Y]=\Pi_{\mathrm{D}_{\mathfrak{k}}} c_{\alpha}^{q_{\alpha}}[X, Y],\left[\sigma_{\mathfrak{p}} X, \sigma_{\mathfrak{p}} Y\right]=c_{\gamma} c_{\delta} \Pi_{D_{\mathfrak{k}}} c_{\alpha}^{p_{\alpha}}[X, Y] .
$$

Therefore, $\sigma_{\mathfrak{k}}[X, Y]=\left[\sigma_{\mathfrak{p}} X, \sigma_{\mathfrak{p}} Y\right]$ if and only if

$$
c_{\gamma} c_{\delta} \Pi_{\mathrm{D}_{\mathfrak{k}}} c_{\alpha}^{p_{\alpha}-q_{\alpha}}=1 .
$$

In all three cases of $\mathfrak{g}_{0}$ in (2.2), $\gamma+\delta=r \sum_{\text {black }} a_{\alpha} \alpha$. Then

$$
\left.\begin{array}{c}
\gamma+\delta+\sum_{\mathrm{D}_{\mathfrak{k}}}\left(p_{\alpha}-q_{\alpha}\right) \alpha=0 \\
r \sum_{\mathrm{D}^{r}} a_{\alpha} \alpha=0 \\
\gamma+\delta=r \sum_{\text {black }} a_{\alpha} \alpha
\end{array}\right\} \Longrightarrow p_{\alpha}-q_{\alpha}=r a_{\alpha} \text { for all } \alpha \in \mathrm{D}_{\mathfrak{k}} .
$$

So (4.12) becomes $\Pi_{\mathrm{P}^{r}} c_{\alpha}^{r a_{\alpha}}=1$, which is precisely condition (1.5) for an admissible marking. This proves the theorem for (4.9) (a).

Next we consider (4.9) (b). We first assume that $\mathrm{D}^{r} \neq A_{2 n}^{1}$. Write $(c, d)=$ $(c, 1) \cdot(1, d)$ as in (4.1). Checking through the painted diagrams in Section 3, we see that the special $d$-orbits satisfy $\Pi_{\beta \in S}(-1)^{a_{\beta}}=1$ (except for $A_{2 n}^{1}$ ), so $(1, d)$ is admissible. Then $\Pi_{\beta \in S}(-1)^{a_{\beta}} \Pi_{\alpha \in \mathrm{P}^{r}} c_{\alpha}^{r a_{\alpha}}=\Pi_{\alpha \in \mathrm{P}^{r}} c_{\alpha}^{r a_{\alpha}}$, namely $(c, d)$ is admissible if and only if $(c, 1)$ is admissible. By Theorem 4.6, $(1, d)$ represents a $\mathfrak{g}_{0}$-involution $\sigma_{d}$. Hence $(c, d)$ represents $\sigma_{c} \sigma_{d}$ if and only if $(c, 1)$ represents $\sigma_{c}$. We conclude that

$$
\begin{aligned}
& (c, d) \text { is admissible } \\
& \Longleftrightarrow(c, 1) \text { is admissible } \\
& \Longleftrightarrow(c, 1) \text { represents a finite order } \mathfrak{g}_{0} \text {-automorphism by (4.9) (a) } \\
& \Longleftrightarrow(c, d) \text { represents a finite order } \mathfrak{g}_{0} \text {-automorphism. }
\end{aligned}
$$

Now consider (4.9) (b) for $\mathrm{D}^{r}=A_{2 n}^{1}$. The painted diagram, along with $d$, is given below. Here $\beta$ is a special $d$-orbit, and $d \gamma=\gamma$.

Write $(c, d)=\left(c^{\prime}, 1\right) \cdot\left(c^{\prime \prime}, d\right)$, where

$$
c_{\alpha}^{\prime}=c_{\alpha} \text { and } c_{\alpha}^{\prime \prime}=1 \text { for all } \alpha \neq \gamma ; c_{\gamma}^{\prime}=-c_{\gamma}, c_{\gamma}^{\prime \prime}=-1 .
$$




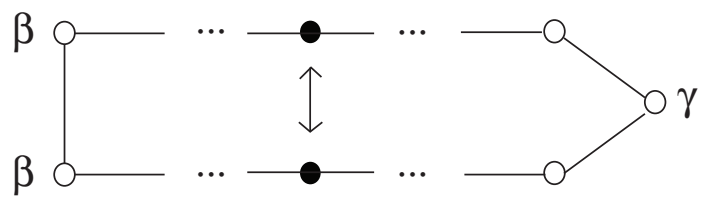

Here $\left(c^{\prime \prime}, d\right)$ is admissible because $(-1)^{a_{\beta}}\left(c_{\gamma}^{\prime \prime}\right)^{a_{\gamma}}=(-1)(-1)=1$. It follows that $(-1)^{a_{\beta}} \prod_{\alpha \in \mathrm{P}^{1}} c_{\alpha}^{r a_{\alpha}}=\Pi_{\alpha \in \mathrm{P}^{1}}\left(c_{\alpha}^{\prime}\right)^{r a_{\alpha}}$, hence $(c, d)$ is admissible if and only if $\left(c^{\prime}, 1\right)$ is admissible. Also, by Theorem [4.6, $\left(c^{\prime \prime}, d\right)$ represents a $\mathfrak{g}_{0}$-involution. Hence $(c, d)$ represents a finite order automorphism if and only if $\left(c^{\prime}, 1\right)$ represents a finite order automorphism. By (4.9) (a), $\left(c^{\prime}, 1\right)$ satisfies this theorem. By repeating the arguments in (4.13) for $(c, d)$ and $\left(c^{\prime}, 1\right)$, we see that $(c, d)$ also satisfies this theorem. This proves the theorem for (4.9) (b).

Finally we consider (4.9) (c). It only happens on $D_{4}^{1}$ with $\operatorname{ord}(d)=3$ and on $D_{2 p}^{1}$ with $\operatorname{ord}(d)=4$. Again write $(c, d)=(c, 1) \cdot(1, d)$ as in (4.1). Here $d$ is the composite of several diagram involutions $d_{i}$. Each $d_{i}$ has no special orbit, so $\left(1, d_{i}\right)$ is admissible. By (4.9) (b), each $\left(1, d_{i}\right)$ represents a $\mathfrak{g}_{0}$-involution $\sigma_{i}$. Then $(1, d)$ is admissible and represents the composite of these $\sigma_{i}$. By (4.9) (a), $(c, 1)$ satisfies this theorem. By repeating the arguments in (4.13), we see that $(c, d)$ also satisfies this theorem. This proves the theorem for (4.9) (c).

We now prove Theorem 1.3. It says that an admissible marking on $\mathrm{P}^{r}$ represents a unique finite order $\mathfrak{g}_{0}$-automorphism, and conversely such an automorphism is represented by an admissible marking. Consequently it uses admissible markings to compute the orders of $\mathfrak{g}_{0}$-automorphisms.

Proof of Theorem 1.3. By Corollary 2.2. a painted diagram $\mathrm{P}^{r}$ represents $\mathfrak{g}_{0}=$ $\mathfrak{k}_{0}+\mathfrak{p}_{0}$, and its white vertices $\mathrm{D}_{\mathfrak{k}} \subset \mathrm{P}^{r}$ is the Dynkin diagram of $\left[\mathfrak{k}_{0}, \mathfrak{k}_{0}\right]$.

Let $(c, d)$ be an admissible marking on $\mathrm{P}^{r}$. By Theorem 4.7, it represents a finite order $\mathfrak{g}_{0}$-automorphism $\sigma$. We prove uniqueness of $\sigma$. By Corollary 2.4, $\sigma$ preserves $\mathfrak{k}$ and $\mathfrak{p}$. Write $\mathfrak{k}=[\mathfrak{k}, \mathfrak{k}]+\mathfrak{z}$. By Corollary 4.3, the restriction of $(c, d)$ to $\mathrm{D}_{\mathfrak{k}}$ uniquely determines $\sigma$ on $[\mathfrak{k}, \mathfrak{k}]$. Since the black vertices of $\mathrm{P}^{r}$ are the lowest weights of $\mathfrak{p}$, the marking also uniquely determines $\sigma$ on the lowest weight spaces of $\mathfrak{p}$, and hence determines $\sigma$ on the entire $\mathfrak{p}$. Further, Proposition 4.5 says that $\sigma$ on $\mathfrak{z}$ is also determined. This proves the uniqueness of $\sigma$.

Recall that in Definition 1.2, for $\alpha \in \mathrm{P}^{r}$, we define $\mathfrak{g}_{\alpha}^{1}=\mathfrak{g}_{\alpha} \cap \mathfrak{k}$ if $\alpha$ is white and $\mathfrak{g}_{\alpha}^{1}=\mathfrak{g}_{\alpha} \cap \mathfrak{p}$ if $\alpha$ is black.

Next we compute $\operatorname{ord}(\sigma)$. Let $\left\{X_{\alpha} \in \mathfrak{g}_{\alpha}^{1}\right\}_{\mathrm{P}^{r}}$ be the root vectors in Definition 1.2. By (4.2), if $\mathcal{O}$ is a $d$-orbit containing $\gamma$, then $\operatorname{ord}\left(\sigma, X_{\gamma}\right)=|\mathcal{O}| \operatorname{ord}\left(\Pi_{\alpha \in \mathcal{O}} c_{\alpha}\right)$. Therefore, $\operatorname{ord}(\sigma)=\operatorname{lcm}_{\mathcal{O}}\left\{\operatorname{ord}\left(\sigma, X_{\alpha}\right)\right\}_{\alpha \in \mathcal{O}}=\operatorname{lcm}_{\mathcal{O}}\left\{|\mathcal{O}| \operatorname{ord}\left(\Pi_{\alpha \in \mathcal{O}} c_{\alpha}\right)\right\}$.

Conversely, let $\sigma$ be a finite order $\mathfrak{g}_{0}$-automorphism. By Corollary 2.4, $\sigma$ preserves $\mathfrak{k}$ and $\mathfrak{p}$. By Corollary 4.3, the restriction of $\sigma$ to $\mathfrak{k}$ is represented by a marking $(c, d)$ on $\mathrm{D}_{\mathfrak{k}}$. By Proposition 4.4 $d$ extends to aut $\left(\mathrm{P}^{r}\right)$. Hence $\sigma \mathfrak{g}_{\alpha}^{1}=\mathfrak{g}_{d \alpha}^{1}$ for all $\alpha \in \mathrm{P}^{r}$. We next extend $c$ to $\mathrm{P}^{r}$ so that $(c, d)$ represents $\sigma$. We divide into two cases:

(a) $\mathrm{D}^{r}=A_{2 n}^{1}$ with $d \neq 1$; (b) remaining situations.

For (4.15) (a), we refer to Figure 4 in the proof of Theorem 4.7. Let $c^{\prime \prime}$ be as given in (4.14). Then $\left(c^{\prime \prime}, d\right)$ is admissible. By Theorem 4.7. $\left(c^{\prime \prime}, d\right)$ represents a 
$\mathfrak{g}_{0}$-involution $\tau$, namely there exist $\left\{X_{\alpha} \in \mathfrak{g}_{\alpha}^{1}\right\}_{\mathrm{P}^{r}}$ such that $\tau X_{\alpha}=X_{d \alpha}$ for all $\alpha \in \mathrm{P}^{r} \backslash \gamma$, and $\tau X_{\gamma}=-X_{\gamma}$. Define $c^{\prime}$ by $\sigma X_{\alpha}=c_{d \alpha}^{\prime} X_{d \alpha}$ for all $\alpha \in \mathrm{P}^{r} \backslash \gamma$, and $\sigma X_{\gamma}=-c_{\gamma}^{\prime} X_{\gamma}$. Then $\left(c^{\prime} c^{\prime \prime}, d\right)$ represents $\sigma$.

For (4.15) (b), the list of all painted diagrams in Section 3 shows that $(1, d)$ is admissible. By Theorem 4.7, $(1, d)$ represents a $\mathfrak{g}_{0}$-automorphism $\sigma_{d}$. So there exist $\left\{X_{\alpha} \in \mathfrak{g}_{\alpha}^{1}\right\}_{\mathrm{P} r}$ such that $\sigma_{d} X_{\alpha}=X_{d \alpha}$ for all $\alpha \in \mathrm{P}^{r}$. Define $c_{\alpha}$ by $\sigma X_{\alpha}=c_{d \alpha} X_{d \alpha}$; then $(c, d)$ represents $\sigma$.

We have shown that in both cases of (4.15) the marking $(c, d)$ on $\mathrm{D}_{\mathfrak{k}}$ extends to $\mathrm{P}^{r}$ and represents $\sigma$. Similar to the arguments in Theorem 4.2. for the black vertices $\beta$ of $\mathrm{P}^{r}$, we may renormalize $X_{\beta}$ so that $c_{\beta} \in U$. By Theorem 4.7 $(c, d)$ is admissible. This proves the theorem.

\section{Equivalent MARKINGS}

In this section, we prove Theorem 1.4. It judges markings on $\mathrm{P}^{r}$ which represent finite order $\mathfrak{g}_{0}$-automorphisms up to conjugation by $\operatorname{int}\left(\mathfrak{g}_{0}\right)$ or aut $\left(\mathfrak{g}_{0}\right)$.

A trivial case is provided by markings $(c, d)$ and $\left(c^{\prime}, d^{\prime}\right)$ which differ merely by a diagram symmetry $f$, namely

$$
c_{f \alpha}=c_{\alpha}^{\prime}, d f \alpha=f d^{\prime} \alpha .
$$

However, there are other situations which may lead to conjugate automorphisms.

Let $(c, d)$ be an admissible marking on $\mathrm{P}^{r}$, and let $\alpha \in \mathrm{D}_{\mathfrak{k}}$. We define the reflection algorithm $F_{\alpha}$ which transforms $(c, d)$ to another marking $\left(F_{\alpha} c, d\right)$ as follows. Given $\beta \in \mathrm{P}^{r}$, let

$$
\left(F_{\alpha} c\right)_{\beta}= \begin{cases}c_{\alpha}^{-1} & \text { if } \beta=\alpha \\ c_{\alpha} c_{\beta} & \text { if } \alpha, \beta \text { are adjacent and }|\alpha| \geq|\beta| \\ c_{\alpha}^{s} c_{\beta} & \text { if } \beta \text { is a longer root joint to } \alpha \text { by an } s \text {-edge } \\ c_{\beta} & \text { otherwise. }\end{cases}
$$

Here $|\alpha|$ and $|\beta|$ are the root lengths.

Let $\sigma$ be a finite order $\mathfrak{g}_{0}$-automorphism. Apply Corollary 2.4(a) to $\Gamma=\langle\sigma\rangle$; there exists a $\sigma$-stable Cartan decomposition $\mathfrak{g}_{0}=\mathfrak{k}_{0}+\mathfrak{p}_{0}$. By Proposition 2.5. there exists a $\sigma$-stable Cartan subalgebra $\mathfrak{h}$ of $\mathfrak{k}$. Let $\Delta=\Delta(\mathfrak{g}, \mathfrak{h})$. The $\mathfrak{k}$-roots $\Delta(\mathfrak{k}, \mathfrak{h})$ have a $\sigma$-stable simple system $\Pi_{\mathfrak{k}}$. The vertices of $D_{\mathfrak{k}}$ represent $\Pi_{\mathfrak{k}}$, and the vertices of $\mathrm{P}^{r}$ represent $\Pi=\Pi_{\mathfrak{k}} \cup$ \{lowest weights of $\left.\mathfrak{p}\right\} \subset \Delta$.

Given $\alpha \in \mathrm{D}_{\mathfrak{k}}$, let $r_{\alpha}: \Delta \longrightarrow \Delta$ be the reflection which maps $\alpha$ to $-\alpha$ and fixes $\alpha^{\perp}$. The following proposition describes the effect of $r_{\alpha}$ on the marking with $d=1$.

Proposition 5.1. Let $(c, 1)$ be a marking on $\mathrm{P}^{r}$ which represents $\sigma$ with respect to $\Pi$. Let $\alpha \in \mathrm{D}_{\mathfrak{k}}$. Then the marking which represents $\sigma$ with respect to $r_{\alpha} \Pi$ is $\left(F_{\alpha} c, 1\right)$.

Proof. Let $\left(c^{\prime}, 1\right)$ be the marking which represents $\sigma$ with respect to $r_{\alpha} \Pi$. Let $\beta \in \mathrm{P}^{r}$. We claim that $c_{\beta}^{\prime}$ is given by (5.2).

Case 1: $r=1$.

Here $\mathfrak{k}$ and $\mathfrak{g}$ have equal rank, and each root space $\mathfrak{g}_{\gamma}$ is 1-dimensional. Extend $c$ to all of $\Delta$ by letting $c_{\gamma}$ be the eigenvalue of $\sigma$ on $\mathfrak{g}_{\gamma}$. By Proposition 2.6,

$$
c_{\gamma+\delta}=c_{\gamma} c_{\delta}, \quad c_{-\gamma}=c_{\gamma}^{-1} .
$$


There are several situations for $\beta$ :

(a) $\beta=\alpha$;

(b) $\beta$ is adjacent to $\alpha$;

(c) $\beta$ and $\alpha$ are distinct and not adjacent.

In (5.4) (a), $c_{\alpha}^{\prime}=c_{r_{\alpha} \alpha}=c_{-\alpha}=c_{\alpha}^{-1}$ by (5.3). This is given by the first line of (5.2). In (5.4) (c), $\beta$ is perpendicular to $\alpha$. Hence $c_{\beta}^{\prime}=c_{r_{\alpha} \beta}=c_{\beta}$. This is given by the last line of (5.2).

It remains to consider $c_{\beta}^{\prime}$ in (5.4)(b). Depending on the root lengths of $\alpha$ and $\beta$, there are several cases as given in Proposition 2.7. In each case, Proposition 2.7 and (5.3) show that $c_{\beta}^{\prime}$ satisfies (5.2). For example, if $|\alpha|=|\beta|$, then Proposition 2.7(a) and (5.3) say that $c_{\beta}^{\prime}=c_{r_{\alpha} \beta}=c_{\alpha+\beta}=c_{\alpha} c_{\beta}$. Similarly, if $\alpha$ is a shorter root joint to $\beta$ by a double edge, then Proposition 2.7(c) and (5.3) say that $c_{\beta}^{\prime}=c_{r_{\alpha} \beta}=$ $c_{2 \alpha+\beta}=c_{\alpha}^{2} c_{\beta}$. The other cases in Proposition 2.7 are handled similarly and are given by the second and third line of (5.2). This proves the proposition for $r=1$.

Case 2: $r=2$.

As explained after Proposition 2.6. the complication comes from the fact that $\mathfrak{g}_{\gamma}$ can be 2-dimensional, and it intersects both $\mathfrak{k}$ and $\mathfrak{p}$ nontrivially. Nevertheless we can repeat the arguments with some modification. We now sketch the method.

Let $\mathbb{Z}_{2}=\{\overline{0}, \overline{1}\}$. Write $\mathfrak{g}=\mathfrak{k}+\mathfrak{p}=\mathfrak{g}^{\overline{0}}+\mathfrak{g}^{\overline{1}}$ so that $\left[\mathfrak{g}^{a}, \mathfrak{g}^{b}\right] \subset \mathfrak{g}^{a+b}$. Let $c_{(\gamma, a)}$ be the eigenvalue of $\sigma$ on $\mathfrak{g}_{\gamma} \cap \mathfrak{g}^{a}$. For example, if $\gamma \in \Pi$ is a black vertex, then $c_{(\gamma, \overline{1})}=c_{\gamma}$. Then (5.3) becomes $c_{(\gamma+\delta, a+b)}=c_{(\gamma, a)} c_{(\delta, b)}$ and $c_{(-\gamma, a)}=c_{(\gamma, a)}^{-1}$. Since $\alpha \in \mathrm{D}_{\mathfrak{k}}$, we have $c_{(\beta, a)}^{\prime}=c_{\left(r_{\alpha} \beta, a\right)}$ for all $\beta \in \Pi$. We then apply similar arguments as in Case 1. For example, let $\beta$ be joint to $\alpha$ by a single edge. Let $a=\overline{0}$ if $\beta$ is white and $a=\overline{1}$ if $\beta$ is black. Then

$$
c_{\beta}^{\prime}=c_{(\beta, a)}^{\prime}=c_{\left(r_{\alpha} \beta, a\right)}=c_{(\alpha+\beta, a)}=c_{(\alpha, \overline{0})} c_{(\beta, a)}=c_{\alpha} c_{\beta} .
$$

The other cases follow similarly.

Corollary 5.2. Let $(c, d)$ be a marking on $\mathrm{P}^{r}$ which represents $\sigma$ with respect to $\Pi$. Let $\alpha_{1}, \ldots, \alpha_{n} \in \mathrm{D}_{\mathfrak{k}}$, and let $\Pi^{\prime}=r_{\alpha_{n}} \cdot \ldots \cdot r_{\alpha_{1}} \Pi$. If $\Pi^{\prime}$ is $\sigma$-stable, then a marking which represents $\sigma$ with respect to $\Pi^{\prime}$ is $\left(F_{\alpha_{n}} \cdot \ldots \cdot F_{\alpha_{1}} c, d\right)$.

Proof. If $d=1$, it follows directly from Proposition 5.1. So we consider $d \neq 1$. We may assume that $\operatorname{ord}(d)=2$, since the case of ord $(d)>2$ for $D_{2 p}^{1}$ can be studied by decomposing $d=d_{1} \cdot d_{2}$, where $\operatorname{ord}\left(d_{i}\right)=2$.

Suppose that $\mathrm{D}^{r} \neq A_{2 m}^{1}$, namely $\mathfrak{g}_{0}$ is not $\mathfrak{s u}(p, q)$ with $p+q$ odd. Let $(c, d)$ represent $\sigma$ with respect to $\Pi$. As discussed in (4.15), for $\mathrm{D}^{r} \neq A_{2 m}^{1},(c, 1)$ and $(1, d)$ are both admissible. So they represent some $\sigma_{c}$ and $\sigma_{d}$, respectively, and $\sigma=\sigma_{c} \cdot \sigma_{d}$. Let $\alpha_{1}, \ldots, \alpha_{n} \in \mathrm{D}_{\mathfrak{k}}$, and let $\Pi^{\prime}=r_{\alpha_{n}} \cdot \ldots \cdot r_{\alpha_{1}} \Pi$ be $\sigma$-stable. We claim that

(a) $\left(F_{\alpha_{n}} \cdot \ldots \cdot F_{\alpha_{1}} c, 1\right)$ represents $\sigma_{c}$ with respect to $\Pi^{\prime}$;

(b) $(1, d)$ represents $\sigma_{d}$ with respect to $\Pi^{\prime}$.

Proposition 5.1 implies (5.5) (a). We now prove (5.5) (b). Since $\Pi^{\prime}$ is $\sigma$-stable, it is also $\sigma_{d}$-stable because $\sigma_{c}$ preserves each root space. Hence $\sigma_{d}$ is represented by a marking $\left(c^{\prime}, d^{\prime}\right)$ with respect to $\Pi^{\prime}$. We shall choose root vectors of $\Pi^{\prime}$ in Definition 1.2 so that $(1, d)=\left(c^{\prime}, d^{\prime}\right)$. Let the subscripts $(\cdot)_{\mathfrak{k}}$ and $(\cdot)_{\mathfrak{p}}$ denote the restrictions 
of a $\mathfrak{g}$-automorphism to $\mathfrak{k}$ and $\mathfrak{p}$ or the restrictions of a marking on $\mathrm{P}^{r}$ to the white and black vertices. Thus we want to show that

$$
\begin{aligned}
& \text { (a) } \quad(1, d)_{\mathfrak{k}}=\left(c^{\prime}, d^{\prime}\right)_{\mathfrak{k}} ; \\
& \text { (b) }(1, d)_{\mathfrak{p}}=\left(c^{\prime}, d^{\prime}\right)_{\mathfrak{p}} .
\end{aligned}
$$

By aut $(\mathfrak{k})=\operatorname{int}(\mathfrak{k}) \rtimes \operatorname{aut}\left(D_{\mathfrak{k}}\right)$, we see that $\left(\sigma_{d}\right)_{\mathfrak{k}}$ uniquely determines the diagram symmetry on $D_{\mathfrak{k}}$ so that $(d)_{\mathfrak{k}}=\left(d^{\prime}\right)_{\mathfrak{k}}$. Furthermore, according to $(1, d)$ with respect to $\Pi$, the inner component of $\left(\sigma_{d}\right)_{\mathfrak{k}}$ is $1 \in \operatorname{int}(\mathfrak{k})$, hence $\left(c^{\prime}\right)_{\mathfrak{k}}=1$. This proves (5.6) (a). In fact it has been verified directly in [1] that the two Vogan diagrams $(1, d)_{\mathfrak{k}}$ and $\left(c^{\prime}, d^{\prime}\right)_{\mathfrak{k}}$ are equivalent if and only if $(d)_{\mathfrak{k}}=\left(d^{\prime}\right)_{\mathfrak{k}}$ and $\left(c^{\prime}\right)_{\mathfrak{k}}=1$.

Next we check (5.6) (b). Depending on whether $\sigma_{d}$ preserves each irreducible component of $\mathfrak{p}$ or interchanges the two irreducible components, one of the following holds:

(a) both $d$ and $d^{\prime}$ fix the black vertices of $\mathrm{P}^{r}$;

(b) both $d$ and $d^{\prime}$ interchange the two black vertices of $\mathrm{P}^{r}$.

We conclude that $(d)_{\mathfrak{p}}=\left(d^{\prime}\right)_{\mathfrak{p}}$. It remains to show that we can make $\left(c^{\prime}\right)_{\mathfrak{p}}=1$ in both situations of (5.7) (a), (b).

Consider (5.7) (a). Let $\beta$ and $\beta^{\prime}$ be extreme $\mathfrak{p}$-weights that are represented by the same black vertex of $\mathrm{P}^{r}$ with respect to $\Pi$ and $\Pi^{\prime}$, respectively. In fact, $(1, d)$ with respect to $\Pi$ implies that $\operatorname{ord}\left(\sigma_{d}\right)=2$, and hence $c_{\beta^{\prime}}^{\prime}= \pm 1$. But we still need $c_{\beta^{\prime}}^{\prime}=1$ to prove (5.6) (b). According to $(1, d)$ with respect to $\Pi, \sigma_{d}=1$ on $\mathfrak{p}_{\beta}$. Write $\beta^{\prime}=\beta+\sum_{\Pi_{\mathfrak{k}}} r_{\alpha} \alpha$. Since $\sigma_{d}$ preserves $\mathfrak{p}_{\beta}$ and $\mathfrak{p}_{\beta^{\prime}}$, it follows that $r_{\alpha}=r_{d \alpha}$ for all $\alpha \in \Pi_{\mathfrak{k}}$. Let $\left\{X_{\alpha}\right\}_{\Pi}$ be the root vectors in Definition 1.2 for the representation of $\sigma_{d}$ by $(1, d)$ with respect to $\Pi$. Thus $\sigma_{d} X_{\alpha}=X_{d \alpha}$. A root vector of $\mathfrak{p}_{\beta^{\prime}}$ is given by $X_{\beta^{\prime}}=\left[\ldots\left[\left[X_{\alpha_{1}}, X_{\alpha_{2}}\right], X_{\alpha_{3}}\right], \ldots, X_{\alpha_{m}}\right]$, where each $\alpha \in \Pi_{\mathfrak{k}}$ appears $r_{\alpha}$ times in $\left\{\alpha_{1}, \ldots, \alpha_{m}\right\}, \beta$ appears once in $\left\{\alpha_{1}, \ldots, \alpha_{m}\right\}$, and each 2-element $d$-orbit appears in successive terms as $\alpha_{i}, \alpha_{i+1}$. Then

$$
\begin{aligned}
\sigma_{d} X_{\beta^{\prime}} & =\left[\ldots\left[\left[\sigma_{d} X_{\alpha_{1}}, \sigma_{d} X_{\alpha_{2}}\right], \sigma_{d} X_{\alpha_{3}}\right], \ldots, \sigma_{d} X_{\alpha_{m}}\right] \\
& =\left[\ldots\left[\left[X_{d \alpha_{1}}, X_{d \alpha_{2}}\right], X_{d \alpha_{3}}\right], \ldots, X_{d \alpha_{m}}\right] .
\end{aligned}
$$

We want to remove all the subscripts $d$ from the last term of (5.8). Since $d$ fixes the black vertices, all the painted diagrams in Section 3 show that there is no special $d$-orbit. By [2, Prop. 3.1(a,d)], we can directly remove all the subscripts $d$. For example if $d \alpha_{1}=\alpha_{1}$ and $d \alpha_{2}=\alpha_{3}$, then $\left[\left[X_{d \alpha_{1}}, X_{d \alpha_{2}}\right], X_{d \alpha_{3}}\right]=\left[\left[X_{\alpha_{1}}, X_{\alpha_{3}}\right], X_{\alpha_{2}}\right]=$ $\left[\left[X_{\alpha_{1}}, X_{\alpha_{2}}\right], X_{\alpha_{3}}\right]$. So the last term of (5.8) becomes $\left[\ldots\left[\left[X_{\alpha_{1}}, X_{\alpha_{2}}\right], X_{\alpha_{3}}\right], \ldots, X_{\alpha_{m}}\right]$. Hence $\sigma_{d} X_{\beta^{\prime}}=X_{\beta^{\prime}}$, namely $c_{\beta^{\prime}}^{\prime}=1$. This proves (5.6) (b) in situation (5.7) (a).

Next consider (5.7) (b), where $d$ interchanges the two black vertices of $\mathrm{P}^{r}$. Let $\beta, \gamma \in \Pi^{\prime}$ be represented by the two black vertices. According to $(1, d)$ with respect to $\Pi, \operatorname{ord}\left(\left(\sigma_{d}\right)_{\mathfrak{p}}\right)=2$. By Theorem 1.3, it forces $c_{\beta}^{\prime} c_{\gamma}^{\prime}=1$. According to $\left(c^{\prime}, d\right)$ with respect to $\Pi^{\prime}$, the root vectors $X_{\beta}, X_{\gamma}$ in Definition 1.2 satisfy $\sigma_{d} X_{\beta}=c_{\gamma}^{\prime} X_{\gamma}$ and $\sigma_{d} X_{\gamma}=c_{\beta}^{\prime} X_{\beta}$. Let $X_{\beta}^{\prime}=c_{\beta}^{\prime} X_{\beta}$ and $X_{\gamma}^{\prime}=X_{\gamma}$. Then $\sigma_{d} X_{\beta}^{\prime}=X_{\gamma}^{\prime}$ and $\sigma_{d} X_{\gamma}^{\prime}=X_{\beta}^{\prime}$, so that $\left(\sigma_{d}\right)_{\mathfrak{p}}$ is represented by $(1, d)_{\mathfrak{p}}$ with respect to $\Pi^{\prime}$ and $X_{\beta}^{\prime}, X_{\gamma}^{\prime}$. This proves (5.6) (b) in situation (5.7) (b). The general effect of the renormalization $X_{\alpha} \mapsto X_{\alpha}^{\prime}$ on the marking will be studied systematically in (5.11) (d).

We have proved (5.6), which implies (5.5) (b). By (5.5), $\left(F_{\alpha_{n}} \cdot \ldots \cdot F_{\alpha_{1}} c, d\right)$ represents $\sigma$ with respect to $\Pi^{\prime}$.

If $\mathrm{D}^{r}=A_{2 m}^{1}$, we write $(c, d)=\left(c^{\prime}, 1\right) \cdot\left(c^{\prime \prime}, d\right)$ as in (4.14). Then $\left(c^{\prime}, 1\right)$ and $\left(c^{\prime \prime}, d\right)$ are both admissible, and we proceed by similar arguments. 
Definition 5.3. Write $(c, d) \rightarrow\left(c^{\prime}, d^{\prime}\right)$ if

(a) $\quad d=d^{\prime}$ and $F_{\alpha} c=c^{\prime}$ for some $F_{\alpha}$; or

(b) $d=d^{\prime}$ and $\Pi_{\alpha \in \mathcal{O}} c_{\alpha}=\Pi_{\alpha \in \mathcal{O}} c_{\alpha}^{\prime}$ for each $d$-orbit $\mathcal{O} \subset \mathrm{P}^{r}$; or

(c) they are related by a diagram symmetry $f$ of (5.1).

Write $(c, d) \rightarrow\left(c^{\prime}, d^{\prime}\right)$ if (5.9) (a) or (5.9) (b) holds. We say that $(c, d)$ and $\left(c^{\prime}, d^{\prime}\right)$ are equivalent if there exist markings $\left(c_{i}, d_{i}\right)$ such that

$$
(c, d)=\left(c_{0}, d_{0}\right) \rightarrow\left(c_{1}, d_{1}\right) \rightarrow \ldots \rightarrow\left(c_{n}, d_{n}\right)=\left(c^{\prime}, d^{\prime}\right) .
$$

We say that $(c, d)$ and $\left(c^{\prime}, d^{\prime}\right)$ are inner equivalent if each " - " in (5.10) can be replaced by " $\rightarrow$ ".

We now prove Theorem 1.4, which uses the equivalence relation in Definition 5.3 to judge conjugate $\mathfrak{g}_{0}$-automorphisms.

Proof of Theorem 1.4. Let $\sigma$ be a finite order $\mathfrak{g}_{0}$-automorphism. By Definition 1.2, it is represented by an admissible marking $(c, d)$ on $\mathrm{P}^{r}$ after we choose

(a) a $\sigma$-stable Cartan decomposition $\mathfrak{k}_{0}+\mathfrak{p}_{0}$;

(b) a $\sigma$-stable Cartan subalgebra $\mathfrak{h}$ of $\mathfrak{k}$ which has a $\sigma$-stable simple system;

(c) a $\sigma$-stable simple system $\Pi_{\mathfrak{k}} \subset \mathfrak{h}^{*}$;

(d) root vectors $\left\{X_{\alpha}\right\}_{\mathrm{P}^{r}}$.

We want to study the effect on $(c, d)$ when (5.11) is changed.

Apply Corollary 2.4(a) to $\Gamma=\langle\sigma\rangle$. Then $\mathfrak{k}_{0}+\mathfrak{p}_{0}$ (or equivalently $\theta$ ) is unique in (5.11) (a). By Proposition 2.5, h is unique in (5.11) (b). Thus (5.11) (a), (b) do not affect the marking.

We next show that the changes in (5.11) (c), (d) are described by (5.9). Recall that $\mathfrak{h}$ is a Cartan subalgebra of $\mathfrak{k}, \Delta=\Delta(\mathfrak{g}, \mathfrak{h}), \Pi_{\mathfrak{k}}$ is a simple system of $\Delta(\mathfrak{k}, \mathfrak{h})$ represented by the vertices of $D_{\mathfrak{k}}$, and $\Pi=\Pi_{\mathfrak{k}} \cup\{$ lowest weights of $\mathfrak{p}\}$ is represented by the vertices of $\mathrm{P}^{r}$.

For (5.11) (c), let $\Pi_{\mathfrak{k}}, \Pi_{\mathfrak{k}}^{\prime} \subset \Delta$ be $\sigma$-stable simple systems of $\mathfrak{k}$. By adding the lowest roots of $\mathfrak{p}$, they extend to $\Pi$ and $\Pi^{\prime}$. Let $(c, d)$ and $\left(c^{\prime}, d\right)$ be the markings on $\mathrm{P}^{r}$ which represent $\sigma$ with respect to $\Pi$ and $\Pi^{\prime}$, respectively. Since aut $(\Delta)$ acts transitively on the family of simple systems, there exists $f \in \operatorname{aut}(\Delta)$ such that $f \Pi_{\mathfrak{k}}=\Pi_{\mathfrak{k}}^{\prime}$ and $f \Pi=\Pi^{\prime}$. Let $f_{\mathfrak{k}}$ be the restriction of $f$ to $\Delta(\mathfrak{k}, \mathfrak{h})$. There is a semidirect product aut $(\Delta(\mathfrak{k}, \mathfrak{h}))=W(\mathfrak{k}) \rtimes \operatorname{aut}\left(\mathrm{D}_{\mathfrak{k}}\right)$, where $W(\mathfrak{k})$ is the Weyl group of $\mathfrak{k}$ 4, Chap. X-3, Thm. 3.29]. The Weyl group is generated by simple reflections. So there exist $\alpha_{i} \in \mathrm{D}_{\mathfrak{k}}$ and $h \in \operatorname{aut}\left(\mathrm{D}_{\mathfrak{k}}\right)$ such that $f_{\mathfrak{k}}=r_{\alpha_{n}} \cdot \ldots \cdot r_{\alpha_{1}} \cdot h$. Each reflection $r_{\alpha_{i}}$ extends to aut $(\Delta)$, and $f_{\mathfrak{k}}$ also extends to $f \in \operatorname{aut}(\Delta)$. So the same holds for $h$, namely $h \in \operatorname{aut}(\Delta)$. So $h$ permutes $\Pi_{\mathfrak{k}}$; it also permutes the corresponding lowest weights of $\mathfrak{p}$, namely $h \in$ aut $\left(\mathrm{P}^{r}\right)$. By Corollary 5.2. $c^{\prime}=F_{\alpha_{n}} \cdot \ldots \cdot F_{\alpha_{1}} \cdot h \cdot c$, where $h \cdot c$ is the diagram symmetry (5.1). By Definition 5.3. $(c, d)$ and $\left(c^{\prime}, d\right)$ are equivalent. If in addition we can choose $f_{\mathfrak{k}}$ such that $h=1$ (namely $(c, d)$ and $\left(c^{\prime}, d\right)$ are inner equivalent), then $\Pi_{\mathfrak{k}}, \Pi_{\mathfrak{k}}^{\prime}$ are conjugate by $\operatorname{int}(\mathfrak{k})$ [8, Ch. VII, Thm. 7.8].

Finally, consider the change of root vectors in (5.11)(d). Fix a $\sigma$-stable $\Pi$, represented by the vertices of $\mathrm{P}^{r}$. Let $(c, d)$ and $\left(c^{\prime}, d\right)$ be the markings which represent $\sigma$ with respect to the root vectors $\left\{X_{\alpha}\right\}_{\alpha \in \mathrm{P}^{r}}$ and $\left\{X_{\alpha}^{\prime}\right\}_{\alpha \in \mathrm{P}^{r}}$, respectively, in Definition 1.2. Let $\{\beta, \gamma\}$ be a 2-element $d$-orbit (a similar argument for a $d$-orbit 
of $D_{2 p}^{1}$ with more elements). Then

$$
\sigma X_{\beta}=\sigma_{c} \cdot \sigma_{d} X_{\beta}=\sigma_{c} X_{\gamma}=c_{\gamma} X_{\gamma}, \sigma X_{\gamma}=\sigma_{c} \cdot \sigma_{d} X_{\gamma}=\sigma_{c} X_{\beta}=c_{\beta} X_{\beta} .
$$

Similarly, $\sigma X_{\beta}^{\prime}=c_{\gamma}^{\prime} X_{\gamma}^{\prime}$ and $\sigma X_{\gamma}^{\prime}=c_{\beta}^{\prime} X_{\beta}^{\prime}$. Let $X_{\beta}^{\prime}=a X_{\beta}$ and $X_{\gamma}^{\prime}=b X_{\gamma}$. Then $c_{\beta}=c_{\beta}^{\prime} \frac{a}{b}$ and $c_{\gamma}=c_{\gamma}^{\prime} \frac{b}{a}$, which imply that $c_{\beta} c_{\gamma}=c_{\beta}^{\prime} c_{\gamma}^{\prime}$. So $(c, d)$ and $\left(c^{\prime}, d\right)$ are related by (5.9) (b). By Definition 5.3, they are inner equivalent.

We have shown that $\sigma$ is represented by distinct markings due to different choices in (5.11) if and only if these markings are equivalent. Furthermore, the choices in (5.11) are conjugate by $\operatorname{int}(\mathfrak{g})$ if and only if the markings are inner equivalent. This proves the theorem.

Theorems 1.3 and 1.4 provide a bijective correspondence between the equivalence (resp. inner equivalence) classes of admissible markings on $\mathrm{P}^{r}$ and the finite order $\mathfrak{g}_{0}$-automorphisms up to conjugation by $\operatorname{aut}\left(\mathfrak{g}_{0}\right)$ (resp. $\left.\operatorname{int}\left(\mathfrak{g}_{0}\right)\right)$. In Examples 9.1 and 9.3 we use this method to classify the automorphisms on $\mathfrak{s u}(3,1)$ of order 3 and the outer automorphisms on $\mathfrak{s u}(2,1)$ of order $\leq 6$.

An example of $\mathfrak{g}_{0}$-automorphisms which are conjugate by aut $\left(\mathfrak{g}_{0}\right)$ but not by $\operatorname{int}\left(\mathfrak{g}_{0}\right)$ is illustrated in [2, Ex. 6.2]. It provides two markings which are equivalent but not inner equivalent.

\section{Extensions of AUtomorphisms: $\mathfrak{g}_{0}$ HeRmitian}

Let $\sigma$ be a finite order $\mathfrak{g}_{0}$-automorphism. By Corollary 2.4 we can regard $\sigma$ as an extension from $\operatorname{aut}_{n}\left(\mathfrak{k}_{0}\right)$ to $\operatorname{aut}_{q n}\left(\mathfrak{g}_{0}\right)$ for some positive integers $n$ and $q$. In this section, we study this extension for $\mathfrak{g}_{0}$ of Hermitian type (2.2) (a) and prove Corollary 1.5. We will treat $\mathfrak{g}_{0}$ of non-Hermitian type (2.2) (b), (c) in the next section.

For convenience we drop the subscript 0 and deal with $\mathfrak{k}$ and $\mathfrak{g}$. Let $\sigma \in \operatorname{aut}_{n}(\mathfrak{k})$ be represented by a marking $(c, d)$ on $\mathrm{D}_{\mathfrak{k}}$. Thus $c: \mathrm{D}_{\mathfrak{k}} \longrightarrow U$ and $d \in \operatorname{aut}\left(\mathrm{D}_{\mathfrak{k}}\right)$, where $U$ is the multiplicative group (1.3). Write $\mathfrak{k}=[\mathfrak{k}, \mathfrak{k}]+\mathfrak{z}$. There are four cases to consider, depending on whether $\sigma$ is an inner or outer automorphism on $[\mathfrak{k}, \mathfrak{k}]$ and $\mathfrak{z}$. Applying (4.4) to $[\mathfrak{k}, \mathfrak{k}]$ and $D_{\mathfrak{k}}$, we get

$$
\begin{aligned}
& \text { (a) } \quad \sigma \in \operatorname{int}([\mathfrak{k}, \mathfrak{k}]) \Longleftrightarrow d=1 \text { on } \mathrm{D}_{\mathfrak{k}} \text {; } \\
& \text { (b) } \sigma \in \operatorname{out}([\mathfrak{k}, \mathfrak{k}]) \Longleftrightarrow d \neq 1 \text { on } \mathrm{D}_{\mathfrak{k}} \text {. }
\end{aligned}
$$

The painted diagram $\mathrm{P}^{1}$ has two black vertices $\{\gamma, \delta\}$. By Proposition 4.5.

$$
\begin{aligned}
& \text { (a) } \sigma \in \operatorname{int}(\mathfrak{z}) \quad \Longleftrightarrow \quad d \text { fixes } \gamma, \delta ; \\
& \text { (b) } \sigma \in \operatorname{out}(\mathfrak{z}) \Longleftrightarrow d \text { interchanges } \gamma, \delta .
\end{aligned}
$$

By (1.7), let $w=\Pi_{\beta \in S_{\mathfrak{k}}}(-1)^{a_{\beta}} \Pi_{\alpha \in \mathrm{D}_{\mathfrak{k}}} c_{\alpha}^{a_{\alpha}}$. The black vertices satisfy $a_{\gamma}=a_{\delta}=1$. By (1.5),$(c, d)$ extends to an admissible marking on $\mathrm{P}^{1}$ if and only if $d$ extends to aut $\left(\mathrm{P}^{1}\right)$, and

$$
\begin{array}{ll}
\text { (a) } \quad c_{\gamma} c_{\delta} w=1 & \text { if }\{\gamma, \delta\} \text { is a not a special } d \text {-orbit; } \\
\text { (b) } c_{\gamma} c_{\delta} w=-1 & \text { if }\{\gamma, \delta\} \text { is a special } d \text {-orbit. }
\end{array}
$$

By Theorem 1.3. if $(c, d)$ extends to an admissible marking, then it represents some $\sigma \in \operatorname{aut}_{m}(\mathfrak{g})$, where

(a) $m=\operatorname{lcm}\left\{n, \operatorname{ord}\left(c_{\gamma}\right), \operatorname{ord}\left(c_{\delta}\right)\right\} \quad$ if $d$ fixes $\gamma$ and $\delta$;

(b) $m=\operatorname{lcm}\left\{n, 2 \operatorname{ord}\left(c_{\gamma} c_{\delta}\right)\right\} \quad$ if $d$ interchanges $\gamma$ and $\delta$. 
We now prove Corollary 1.5, which describes the extensions of $\sigma \in \operatorname{aut}_{n}\left(\mathfrak{k}_{0}\right)$ to $\operatorname{aut}_{q n}\left(\mathfrak{g}_{0}\right)$ for $\mathfrak{g}_{0}$ of Hermitian type.

Proof of Corollary 1.5. Let $\sigma \in \operatorname{aut}_{n}(\mathfrak{k})$ be represented by a marking $(c, d)$ on $\mathrm{D}_{\mathfrak{k}}$. We consider its extension to $\operatorname{aut}_{m}(\mathfrak{g})$. It is necessary that $n$ divides $m$ and also that $d$ extends to aut $\left(\mathrm{P}^{r}\right)$, by Proposition 4.4 So let us assume these conditions. We divide the proof into four cases, depending on whether $\sigma$ is inner or outer on $[\mathfrak{k}, \mathfrak{k}]$ and $\mathfrak{z}$.

Suppose that $\sigma \in \operatorname{int}(\mathfrak{k})$. By (6.1) (a) and (6.2) (a), $d=1$ on $\mathrm{D}_{\mathfrak{k}}$, and we extend $(c, d)$ to an admissible marking on $\mathrm{P}^{1}$ of order $m$ with $d=1$. So we want $c_{\gamma}, c_{\delta}$ to satisfy (6.3) (a) and (6.4)(a). They can always be achieved, for instance, by $c_{\gamma}=\exp \frac{2 \pi i}{m}$ and $c_{\delta}=\exp \frac{-2 \pi i}{m} w^{-1}$. This proves Corollary [1.5(a).

Suppose that $\sigma \in \operatorname{int}([\mathfrak{k}, \mathfrak{k}]) \oplus \operatorname{out}(\mathfrak{z})$. By (6.1) (a) and (6.2) (b), we look for admissible markings $(c, d)$ on $\mathrm{P}^{1}$ such that $d=1$ on $\mathrm{D}_{\mathfrak{k}}$ and that $d$ interchanges $\gamma, \delta$. In Figure 1 of Section 3, the only painted diagrams which allow such $d$ are $\mathfrak{s u}(2,2)$ (a loop with four vertices, and opposite colors on adjacent vertices), $\mathfrak{s p}(2, \mathbb{R})$ and $\mathfrak{s o}(2, q)$. In these cases,

$$
\begin{array}{rlrl}
m & =\operatorname{lcm}\left\{n, 2 \operatorname{ord}\left(c_{\gamma} c_{\delta}\right)\right\} & \text { by }(6.4)(\mathrm{b}) \\
& =\operatorname{lcm}\left\{n, 2 \operatorname{ord}\left(c_{\gamma} c_{\gamma}^{-1} w^{-1}\right)\right\} & \text { by (6.3) }(\mathrm{a}) \\
& =\operatorname{lcm}\{n, 2 \operatorname{ord}(w)\} . & &
\end{array}
$$

This proves Corollary 1.5(b).

Suppose that $\sigma \in \operatorname{out}([\mathfrak{k}, \mathfrak{k}]) \oplus \operatorname{int}(\mathfrak{z})$. By $(6.1)(b)$ and $(6.2)(a)$, we look for admissible markings $(c, d)$ on $\mathrm{P}^{1}$ such that $d \neq 1$ on $\mathrm{D}_{\mathfrak{k}}$, and that $d$ fixes $\gamma, \delta$. In Figure 1 of Section 3, the only painted diagrams which allow such $d$ are $\mathfrak{s u}(p, p)$ and $\mathfrak{s o}(2,2 q)$. In these two cases,

$$
\begin{array}{rlrl}
m & =\operatorname{lcm}\left\{n, \operatorname{ord}\left(c_{\gamma}\right), \operatorname{ord}\left(c_{\delta}\right)\right\} & & \text { by }(6.4)(\mathrm{a}) \\
& =\operatorname{lcm}\left\{n, \operatorname{ord}\left(c_{\gamma}\right), \operatorname{ord}\left(c_{\gamma}^{-1} w^{-1}\right)\right\} & \text { by (6.3) (a). }
\end{array}
$$

This can always be satisfied, for example, by $c_{\gamma}=\exp \frac{2 \pi i}{m}$ and $c_{\delta}=\exp \frac{-2 \pi i}{m} w^{-1}$. This proves Corollary 1.5(c).

Finally suppose that $\sigma \in \operatorname{out}([\mathfrak{k}, \mathfrak{k}]) \oplus \operatorname{out}(\mathfrak{z})$. By (6.1) (b) and (6.2) (b), we look for admissible markings $(c, d)$ on $\mathrm{P}^{1}$ such that $d$ acts nontrivially on the white vertices as well as the black vertices. For $\mathfrak{g}_{0} \neq \mathfrak{s u}(1, q)$, the black vertices are not adjacent, so we repeat (6.5) and get $m=\operatorname{lcm}\{n, 2 \operatorname{ord}(w)\}$. For $\mathfrak{s u}(1, q)$, the black vertices are adjacent, so

$$
\begin{aligned}
m & =\operatorname{lcm}\left\{n, 2 \operatorname{ord}\left(c_{\gamma} c_{\delta}\right)\right\} & \text { by }(\underline{6.4)})(\mathrm{b}) \\
& =\operatorname{lcm}\left\{n, 2 \operatorname{ord}\left(c_{\gamma}\left(-c_{\gamma}^{-1} w^{-1}\right)\right)\right\} & \text { by }(6.3)(\mathrm{b}) \\
& =\operatorname{lcm}\{n, 2 \operatorname{ord}(-w)\} . &
\end{aligned}
$$

This proves Corollary $1.5(\mathrm{~d})$.

\section{Extensions of AUTOMORPHiSMS: $\mathfrak{g}_{0}$ NON-HERMitian}

Let $\mathfrak{g}_{0}$ be of non-Hermitian type (2.2) (b), (c). In this section, we prove Corollary 1.6. Just as in the previous section, by Corollary 2.4, we may complexify and work on the extension from $\mathfrak{k}$ to $\mathfrak{g}$.

The next proposition and corollary impose a restriction on the order of the extension of $\sigma$ to $\mathfrak{g}$. The Cartan involution $\theta$ of $\mathfrak{g}_{0}$ satisfies $\theta= \pm 1$ on $\mathfrak{k}$ and $\mathfrak{p}$. 
Proposition 7.1. Suppose that $\mathfrak{g}_{0}$ is of non-Hermitian type. The identity map on $\mathfrak{k}$ has only two extensions to $\mathfrak{g}$, namely 1 and $\theta$.

Proof. Suppose that the identity map $1 \in \operatorname{aut}(\mathfrak{k})$ extends to $\tilde{1} \in \operatorname{aut}(\mathfrak{g})$. The unique black vertex $\gamma$ of $\mathrm{P}^{r}$ is the lowest weight of $\mathfrak{p}$. Since $\tilde{1}$ preserves the simple root spaces of $\mathfrak{k}$, it also preserves the lowest weight space $\mathfrak{p}_{\gamma}$. Hence $\tilde{1}$ acts as multiplication by $z \in \mathbb{C}$ on $\mathfrak{p}_{\gamma}$. Given a vector of the form $[X, Y] \in \mathfrak{p}$ where $X \in \mathfrak{k}$ and $Y \in \mathfrak{p}_{\gamma}$, we have $\tilde{1}[X, Y]=[\tilde{1} X, \tilde{1} Y]=[X, z Y]=z[X, Y]$. Hence $\tilde{1}$ acts as multiplication by $z$ on $[X, Y]$. Since $\mathfrak{p}_{\gamma}$ is the lowest weight space, we continue this argument on the higher weight spaces of $\mathfrak{p}$ and conclude that $\tilde{1}$ acts as multiplication by $z$ on the entire $\mathfrak{p}$.

Since $\mathfrak{g}$ is simple and $[\mathfrak{p}, \mathfrak{p}]+\mathfrak{p}$ is an ideal of $\mathfrak{g}$, we get $[\mathfrak{p}, \mathfrak{p}]=\mathfrak{k}$. Pick $[X, Y] \in \mathfrak{k}$, where $X, Y \in \mathfrak{p}$. Then $[X, Y]=\tilde{1}[X, Y]=[\tilde{1} X, \tilde{1} Y]=[z X, z Y]=z^{2}[X, Y]$. Hence $z^{2}=1$. If $z=1$, then $\tilde{1}$ is the identity map. If $z=-1$, then $\tilde{1}=\theta$.

Corollary 7.2. Suppose that $\mathfrak{g}_{0}$ is of non-Hermitian type. Then any $\sigma \in \operatorname{aut}_{n}(\mathfrak{k})$ may only extend to $\operatorname{aut}_{n}(\mathfrak{g})$ or $\operatorname{aut}_{2 n}(\mathfrak{g})$.

Proof. Let $\tilde{\sigma} \in \operatorname{aut}(\mathfrak{g})$ be an extension of $\sigma \in \operatorname{aut}_{n}(\mathfrak{k})$. Then $\tilde{\sigma}^{n}$ is an extension of $\sigma^{n}=1$. By Proposition 7.1, either $\tilde{\sigma}^{n}=1$ or $\tilde{\sigma}^{n}=\theta$. Therefore, either $\tilde{\sigma} \in \operatorname{aut}_{n}(\mathfrak{g})$ or $\tilde{\sigma} \in \operatorname{aut}_{2 n}(\mathfrak{g})$.

We now prove Corollary 1.6, which describes the extensions of $\sigma \in \operatorname{aut}_{n}\left(\mathfrak{k}_{0}\right)$ to $\operatorname{aut}\left(\mathfrak{g}_{0}\right)$ for $\mathfrak{g}_{0}$ of non-Hermitian type.

Proof of Corollary 1.6. Let $\sigma \in \operatorname{aut}_{n}(\mathfrak{k})$, represented by a marking $(c, d)$ on $\mathrm{D}_{\mathfrak{k}}$. We consider its extension to $\operatorname{aut}_{m}(\mathfrak{g})$. By Corollary 7.2, $m \in\{n, 2 n\}$. Let $\gamma$ be the unique black vertex of $\mathrm{P}^{r}$, so $r a_{\gamma}=2$. Let $w$ be as defined in (1.7). By Proposition 4.4. if $\sigma$ extends to $\mathfrak{g}$, then $d$ extends to $\mathrm{P}^{r}$. Conversely, if $d$ extends to $\mathrm{P}^{r}$, then we get an admissible marking (1.5) by letting $c_{\gamma}$ satisfy

$$
c_{\gamma}^{2} w=1 \text {. }
$$

There are two solutions of $c_{\gamma}$ in (7.1), and by Theorem 1.3, each solution extends $\sigma$ to some $\operatorname{aut}_{m}(\mathfrak{g})$, where

$$
\begin{aligned}
m & =\operatorname{lcm}\left\{n, \operatorname{ord}\left(c_{\gamma}\right)\right\} \\
& =\operatorname{lcm}\left\{n, \operatorname{ord}\left( \pm w^{\frac{-1}{2}}\right)\right\} \quad \text { by (7.1) } \\
& =\operatorname{lcm}\{n, \operatorname{ord}( \pm \sqrt{w})\} .
\end{aligned}
$$

This proves Corollary 1.6

\section{FiXed POINTS OF AUtOMORPHISMS}

This section studies the fixed point set $\mathfrak{g}_{0}^{\sigma}=\left\{X \in \mathfrak{g}_{0} ; \sigma X=X\right\}$. We assume that $\sigma$ is represented by a marking which satisfies (1.8). We shall construct Dynkin diagrams $\mathrm{D}\left(\sigma_{\mathfrak{g}}\right), \mathrm{D}\left(\sigma_{\mathfrak{k}}\right)$ and integers $p, q$, and then prove Theorem 1.7 .

Given $\sigma \in$ aut $_{m}\left(\mathfrak{g}_{0}\right)$, let $\sigma_{\mathfrak{g}}$ be its natural extension to $\mathfrak{g}$. Let $c$ be a marking on $\mathrm{D}^{r}$ which represents $\sigma_{\mathfrak{g}}$. Given another marking $c^{\prime}$ on $\mathrm{D}^{r}$, we say that $c$ and $c^{\prime}$ are $\mathfrak{g}$-equivalent if there exists a sequence

$$
c=c_{1} \longrightarrow c_{2} \longrightarrow \ldots \longrightarrow c_{s}=c^{\prime}
$$

such that each $c_{i} \longrightarrow c_{i+1}$ is given by the formula $F_{\alpha}$ of (5.2), where $\alpha$ may be a white or black vertex. Unlike (5.2), here we allow $\alpha$ to be white or black. So here 
$c$ and $c^{\prime}$ are $\mathfrak{g}$-equivalent if and only if they represent conjugate $\mathfrak{g}$-automorphisms, whereas Definition 5.3 is concerned with conjugate $\mathfrak{g}_{0}$-automorphisms. We say that $c$ is $\mathfrak{g}$-standard if it satisfies the condition of Theorem 2.1, namely $c_{\alpha}=\left(\exp \frac{2 \pi i}{m}\right)^{n_{\alpha}}$, where $n_{\alpha}$ are nonnegative integers without nontrivial common factor such that

$$
r \sum_{\mathrm{D}^{r}} a_{\alpha} n_{\alpha}=m
$$

By Theorem 2.1 $c$ is $\mathfrak{g}$-equivalent to a unique $\mathfrak{g}$-standard marking $c_{\mathfrak{g}}$ on $\mathrm{D}^{r}$ (more explanation in Remark 8.1). Define the subdiagram

$$
\mathrm{D}\left(\sigma_{\mathfrak{g}}\right)=\left\{\alpha \in \mathrm{D}^{r} ;\left(c_{\mathfrak{g}}\right)_{\alpha}=1\right\} .
$$

Let $\sharp(\cdot)$ denote the number of vertices, and let

$$
p=\sharp\left\{\alpha \in \mathrm{D}^{r} ;\left(c_{\mathfrak{g}}\right)_{\alpha} \neq 1\right\}-1 .
$$

The Dynkin diagram of $\mathfrak{k}$ is given by the white vertices $\mathrm{D}_{\mathfrak{k}} \subset \mathrm{P}^{r}$. We tentatively assume that $\mathrm{D}_{\mathfrak{k}}$ is connected. Let $\left(\mathrm{D}_{\mathfrak{k}}\right)^{1}$ be its corresponding affine diagram formed by adding the lowest $\mathfrak{k}$-root $\varphi$ to $D_{\mathfrak{k}}$, and let $\left\{b_{\alpha}\right\}_{\left(D_{\mathfrak{k}}\right)^{1}}$ be the positive integers without nontrivial common factor such that $\sum_{\left(\mathrm{D}_{\mathfrak{k}}\right)^{1}} b_{\alpha} \alpha=0$. This is analogous to (1.1) for $a_{\alpha}$. Let $c_{\varphi}=\Pi_{\mathrm{D}_{\mathfrak{k}}} c_{\alpha}^{-b_{\alpha}}$ so that $\Pi_{\left(\mathrm{D}_{\mathfrak{k}}\right)^{1}} c_{\alpha}^{b_{\alpha}}=1$. If $c^{\prime}$ is another marking on $\left(D_{\mathfrak{k}}\right)^{1}$, we say that $c$ and $c^{\prime}$ are $\mathfrak{k}$-equivalent (as markings on $\left(\mathrm{D}_{\mathfrak{k}}\right)^{1}$ ) if they are related by $c=c_{1} \longrightarrow c_{2} \longrightarrow \ldots \longrightarrow c_{s}=c^{\prime}$, where each $c_{i} \longrightarrow c_{i+1}$ is a reflection algorithm $F_{\alpha}$ for some $\alpha \in\left(\mathrm{D}_{\mathfrak{k}}\right)^{1}$. Similar to the proof of Theorem 1.4, $c$ and $c^{\prime}$ are $\mathfrak{k}$-equivalent if and only if they represent conjugate $\mathfrak{k}$-automorphisms. We say that $c$ is $\mathfrak{k}$-standard if $c_{\alpha}=\exp \frac{2 \pi i k_{\alpha}}{m}$, where $k_{\alpha}$ are nonnegative integers without nontrivial common factor such that

$$
\sum_{\left(D_{\mathfrak{k}}\right)^{1}} b_{\alpha} k_{\alpha}=m .
$$

Let $\sigma_{\mathfrak{k}}$ be the restriction of $\sigma$ to $\mathfrak{k}$. In general, $\operatorname{ord}\left(\sigma_{\mathfrak{k}}\right) \leq \operatorname{ord}(\sigma)=m$. If indeed $\operatorname{ord}\left(\sigma_{\mathfrak{k}}\right)<m$, we replace $m$ by $\operatorname{ord}\left(\sigma_{\mathfrak{k}}\right)$ in (8.5). But to avoid cumbersome notation, let us assume that $\operatorname{ord}\left(\sigma_{\mathfrak{k}}\right)=m$.

By Theorem 2.1, $c$ is $\mathfrak{k}$-equivalent to a unique $\mathfrak{k}$-standard marking $c_{\mathfrak{k}}$ on $\left(\mathrm{D}_{\mathfrak{k}}\right)^{1}$. Define the subdiagram

$$
\mathrm{D}\left(\sigma_{\mathfrak{k}}\right)=\left\{\alpha \in\left(\mathrm{D}_{\mathfrak{k}}\right)^{1} ;\left(c_{\mathfrak{k}}\right)_{\alpha}=1\right\}
$$

If $D_{\mathfrak{k}}$ is not connected, we perform the above construction on every connected component $\kappa$ of $D_{\mathfrak{k}}$. Namely, for each $\kappa$, the above method provides a $\mathfrak{k}$-standard marking $c_{\mathfrak{k}}^{\kappa}$ on $\kappa^{1}$ and hence a subdiagram of $\kappa^{1}$ formed by the vertices $\alpha$ with $\left(c_{\mathfrak{k}}^{\kappa}\right)_{\alpha}=1$. Then let $\mathrm{D}\left(\sigma_{\mathfrak{k}}\right)$ be the union over these components $\kappa$. We also let

$$
q= \begin{cases}\sum_{\kappa}\left(\sharp\left\{\alpha \in \kappa^{1} ;\left(c_{\mathfrak{k}}^{\kappa}\right)_{\alpha} \neq 1\right\}-1\right) & \text { if } \mathfrak{g}_{0} \text { is of non-Hermitian type; } \\ 1+\sum_{\kappa}\left(\sharp\left\{\alpha \in \kappa^{1} ;\left(c_{\mathfrak{k}}^{\kappa}\right)_{\alpha} \neq 1\right\}-1\right) & \text { if } \mathfrak{g}_{0} \text { is of Hermitian type. }\end{cases}
$$

We now prove Theorem 1.7. It uses the diagrams $\mathrm{D}\left(\sigma_{\mathfrak{g}}\right), \mathrm{D}\left(\sigma_{\mathfrak{k}}\right)$ and integers $p, q$ to describe the fixed point set $\mathfrak{g}_{0}^{\sigma}$.

Proof of Theorem 1.7. By Theorem 2.1(b), $\mathfrak{g}^{\sigma}$ is complex reductive, with Dynkin diagram $\mathrm{D}\left(\sigma_{\mathfrak{g}}\right)$ and center of dimension $p$. Also, $\mathfrak{k}^{\sigma}$ has Dynkin diagram $\mathrm{D}\left(\sigma_{\mathfrak{k}}\right)$. We next consider the center of $\mathfrak{k}^{\sigma}$. 
Suppose that $\mathfrak{g}_{0}$ is of non-Hermitian type. Then $\mathfrak{k}$ is semisimple. If $\mathfrak{k}^{\prime}$ is a simple ideal of $\mathfrak{k}$, its Dynkin diagram is a connected component $\kappa$ of $D_{\mathfrak{k}}$. Theorem 2.1(b) says that the center of $\mathfrak{k}^{\sigma} \cap \mathfrak{k}^{\prime}=\left(\mathfrak{k}^{\prime}\right)^{\sigma}$ has dimension $\sharp\left\{\alpha \in \kappa^{1} ;\left(c_{\mathfrak{k}}^{\kappa}\right)_{\alpha} \neq 1\right\}-1$. By (8.7), $\mathfrak{k}^{\sigma}$ has center of dimension $q$.

Suppose that $\mathfrak{g}_{0}$ is of Hermitian type. Then $\mathfrak{k}=[\mathfrak{k}, \mathfrak{k}]+\mathfrak{z}$, where $[\mathfrak{k}, \mathfrak{k}]$ is semisimple and $\mathfrak{z}$ is a one-dimensional center. By the above argument, $[\mathfrak{k}, \mathfrak{k}]^{\sigma}$ has center of dimension $\sum_{\kappa}\left(\sharp\left\{\alpha \in \kappa^{1} ;\left(c_{\mathfrak{k}}^{\kappa}\right)_{\alpha} \neq 1\right\}-1\right)$. The marking on $\mathrm{D}_{\mathfrak{k}}$ has trivial diagram automorphism, so by Proposition 4.5(a), $\mathfrak{z} \subset \mathfrak{k}^{\sigma}$. Hence $\mathfrak{k}^{\sigma}=[\mathfrak{k}, \mathfrak{k}]^{\sigma}+\mathfrak{z}$, and by (8.7), $\mathfrak{k}^{\sigma}$ has center of dimension $q$. We have proved Theorem 1.7(a), (b).

By Theorem 1.7(a),

$$
\operatorname{rank} \mathfrak{g}^{\sigma}=\sharp\left(\mathrm{D}\left(\sigma_{\mathfrak{g}}\right)\right)+p=\sharp\left(\mathrm{D}^{r}\right)-1 .
$$

Suppose that $\mathfrak{g}_{0}$ is of non-Hermitian type. By (2.2), $\sharp\left(\mathrm{D}_{\mathfrak{k}}\right)=\sharp\left(\mathrm{D}^{r}\right)-1$. By Theorem 1.7(b),

$$
\operatorname{rank} \mathfrak{k}^{\sigma}=\sharp\left(\mathrm{D}\left(\sigma_{\mathfrak{k}}\right)\right)+q=\sum_{\kappa} \sharp(\kappa)=\sharp\left(\mathrm{D}_{\mathfrak{k}}\right)=\sharp\left(\mathrm{D}^{r}\right)-1 .
$$

By (8.8) and (8.9), rank $\mathfrak{g}^{\sigma}=\operatorname{rank} \mathfrak{k}^{\sigma}$.

Suppose that $\mathfrak{g}_{0}$ is of Hermitian type. By (2.2), $\sharp\left(D_{\mathfrak{k}}\right)=\sharp\left(\mathrm{D}^{r}\right)-2$, and we replace (8.9) by

$$
\operatorname{rank} \mathfrak{k}^{\sigma}=\sharp\left(\mathrm{D}\left(\sigma_{\mathfrak{k}}\right)\right)+q=1+\sum_{\kappa} \sharp(\kappa)=1+\sharp\left(\mathrm{D}_{\mathfrak{k}}\right)=\sharp\left(\mathrm{D}^{r}\right)-1 .
$$

Once again, rank $\mathfrak{g}^{\sigma}=\operatorname{rank} \mathfrak{k}^{\sigma}$.

We have shown that rank $\mathfrak{g}^{\sigma}=\operatorname{rank} \mathfrak{k}^{\sigma}$, so the center of $\mathfrak{g}_{0}^{\sigma}$ is compact. This proves Theorem 1.7 (c).

A real simple Lie algebra is determined by its complexification and maximal compact subalgebra [4, Ch. X-6, Thm. 6.2]. Although here $\mathfrak{g}_{0}^{\sigma}$ is merely real reductive, Theorem 1.7 gives an effective way to compute $\mathfrak{g}_{0}^{\sigma}$. The condition rank $\mathfrak{g}^{\sigma}=\operatorname{rank} \mathfrak{k}^{\sigma}$ is essential in determining $\mathfrak{g}_{0}^{\sigma}$. For example, suppose that $\mathfrak{g}^{\sigma}=A_{2}+\mathbb{C}$ and $\mathfrak{k}^{\sigma}=A_{1}+\mathbb{C}$. Here rank $\mathfrak{g}^{\sigma}=3$ and rank $\mathfrak{k}^{\sigma}=2$. Then $\mathfrak{g}_{0}^{\sigma}$ can be $\mathfrak{s l}(3, \mathbb{R})+\mathfrak{s o}(2)$ or $\mathfrak{s u}(2,1)+\mathbb{R}$. The notation $\mathfrak{s o}(2) \subset \mathfrak{g}_{0}^{\sigma}$ and $\mathbb{R} \subset \mathfrak{g}_{0}^{\sigma}$ for the center of $\mathfrak{g}_{0}^{\sigma}$ are used to distinguish whether it is contained in $\mathfrak{k}_{0}$ or $\mathfrak{p}_{0}$. We shall work out some examples of $\mathfrak{g}_{0}^{\sigma}$ in the next section.

Remark 8.1. In (8.1) through (8.2), we let $\sigma_{\mathfrak{g}} \in \operatorname{aut}_{m}(\mathfrak{g})$ be represented by $c$, and mention that $c$ is $\mathfrak{g}$-equivalent to a unique $\mathfrak{g}$-standard marking $c_{\mathfrak{g}}$ on $\mathrm{P}^{r}$. We now discuss this in more detail.

Let $\mathcal{C}$ be all the admissible markings on $\mathrm{P}^{r}$ with $d=1$. By (1.5), $c \in \mathcal{C}$ if and only if $\Pi_{\mathrm{P}^{r}} c_{\alpha}^{r a_{\alpha}}=1$, namely

$$
\text { (a) } \Pi_{\mathrm{P}^{1}} c_{\alpha}^{a_{\alpha}}=1 \text { or } \quad \text { (b) } \Pi_{\mathrm{P}^{2}} c_{\alpha}^{a_{\alpha}}= \pm 1 \text {. }
$$

Let $\sigma_{\mathfrak{g}}$ be a finite order $\mathfrak{g}$-automorphism.

If $r=1$, apply Theorem 2.1 and represent $\sigma_{\mathfrak{g}}$ by integers $0 \leq n_{\alpha}<m$ such that $\sum_{\mathrm{P}^{1}} a_{\alpha} n_{\alpha}=m$. Then $\left(c_{\mathfrak{g}}\right)_{\alpha}=\exp \left(\frac{2 \pi i}{m} n_{\alpha}\right)$ is $\mathfrak{g}$-standard and satisfies (8.10) (a). By Theorem 2.1, every $c \in \mathcal{C}$ is $\mathfrak{g}$-equivalent to some $c_{\mathfrak{g}}$ constructed in this way.

The situation is more subtle with $r=2$. By (8.10) (b), define

$$
f: \mathcal{C} \longrightarrow\{ \pm 1\}, \quad f(c)=\Pi_{\mathrm{P}^{2}} c_{\alpha}^{a_{\alpha}} .
$$


Let $\sigma_{\mathfrak{g}}$ be represented by $c$. We claim that

(a) $\sigma_{\mathfrak{g}}$ is inner $\Longrightarrow f(c)=1$;

(b) $\sigma_{\mathfrak{g}}$ is outer $\Longrightarrow f(c)=-1$.

We first prove (8.11) (b). Suppose that $f(c)=1$. Define another marking $\sqrt{c}$ on $\mathrm{P}^{2}$ by $(\sqrt{c})_{\alpha}=\sqrt{c_{\alpha}}$ for all $\alpha \in \mathrm{P}^{2}$. Then $\Pi_{\mathrm{P}^{2}}(\sqrt{c})_{\alpha}^{a_{\alpha}}=-1$, so $\sqrt{c}$ is admissible. It represents a $\mathfrak{g}$-automorphism $\tau$, where

$$
\tau^{2}=\sigma_{\mathfrak{g}} .
$$

We now use (8.12) to show that $\sigma_{\mathfrak{g}}$ is inner. Recall that $\operatorname{aut}(\mathfrak{g}) / \operatorname{int}(\mathfrak{g})=\operatorname{aut}(\mathrm{D})$, where $\mathrm{D}$ is the Dynkin diagram of $\mathfrak{g}$. If $\mathfrak{g} \neq D_{4}$, then aut(D) is trivial or $\mathbb{Z}_{2}$, so (8.12) implies that $\sigma_{\mathfrak{g}}$ is inner. Suppose that $\mathfrak{g}=D_{4}$. Let $\beta$ be the unique black vertex of $\mathrm{P}^{2}$. Let $\theta \in$ aut $_{2}(\mathfrak{g})$ be the Cartan involution on $\mathfrak{g}_{0}$. By Theorem 2.1. we represent $\theta$ by $n_{\beta}=1$ and $n_{\alpha}=0$ for other $\alpha$, so that it coincides with the painting. By (4.4) (b), $\theta$ is outer. The corresponding marking is $c_{\alpha}^{\prime}=\exp \left(i \pi n_{\alpha}\right)$, namely $c_{\beta}=-1$ and $c_{\alpha}=1$ for other $\alpha$. Since $c^{\prime}$ and $\sqrt{c}$ commute, the same is true for $\theta, \tau \in \operatorname{aut}(\mathfrak{g})$, as well as their coset elements $[\theta],[\tau] \in \operatorname{aut}(\mathfrak{g}) / \operatorname{int}(\mathfrak{g})=S_{3}$. Since $\operatorname{ord}([\theta])=2$, the centralizer of $[\theta]$ in $S_{3}$ is $\{1,[\theta]\}$. Hence $[\tau] \in\{1,[\theta]\}$, and therefore $\left[\tau^{2}\right]=1$. By (8.12), $\sigma_{\mathfrak{g}}$ is inner. This proves (8.11) (b).

To prove (8.11) (a), suppose that $\sigma_{\mathfrak{g}}$ is inner. Let $\theta \in$ aut $_{2}(\mathfrak{g})$ be the Cartan involution of $\mathfrak{g}_{0}$. As discussed above it is outer, and it is represented by the marking $c^{\prime}$ with value -1 at the black vertex and 1 elsewhere. Hence $f\left(c^{\prime}\right)=-1$. Then $\sigma_{\mathfrak{g}} \cdot \theta$ is outer and is represented by $c c^{\prime}$. By (8.11) (b), $-1=f\left(c c^{\prime}\right)=f(c) f\left(c^{\prime}\right)=$ $f(c) \cdot(-1)$, which implies that $f(c)=1$. This proves (8.11) (a).

By Theorem 2.1] and (4.4), if $\sigma_{\mathfrak{g}}$ is represented by a marking $c$ on $\mathrm{P}^{2}$, then $c$ is $\mathfrak{g}$-equivalent to a $\mathfrak{g}$-standard marking if and only if $\sigma_{\mathfrak{g}}$ is outer (i.e. for $\sigma_{\mathfrak{g}}$ inner, Theorem 2.1 uses $\mathrm{P}^{1}$ instead of $\left.\mathrm{P}^{2}\right)$. By (8.11), this is equivalent to $\Pi_{\mathrm{P}^{2}} c_{\alpha}^{a_{\alpha}}=-1$, which is precisely the condition in (1.8). We summarize our discussion in the following proposition.

Proposition 8.2. Let $\sigma$ be a finite order $\mathfrak{g}_{0}$-automorphism, represented by a marking $c$ on $\mathrm{P}^{r}$ with $d=1$.

(a) If $r=1$, then $\sigma$ is inner.

(b) Suppose $r=2$. Then $\sigma$ is inner if $\Pi_{\mathrm{P}^{2}} c_{\alpha}^{a_{\alpha}}=1$ and $\sigma$ is outer if $\Pi_{\mathrm{P}^{2}} c_{\alpha}^{a_{\alpha}}=-1$.

In the above discussion, for a finite order $\mathfrak{g}_{0}$-automorphism $\sigma$ represented on $\mathrm{P}^{2}$, we construct $\theta \in \operatorname{aut}_{2}(\mathfrak{g})$ from the Cartan involution such that $\theta$ is outer and commutes with $\sigma$. Thus one of $\{\sigma, \sigma \theta\}$ is inner while the other is outer. So when $\sigma$ is an involution, the associated symmetric pairs of $\mathfrak{g}_{0}$ arising from $\sigma$ and $\sigma \theta$ are not conjugate. Hence in the diagrammatic classification of the symmetric pairs on $A_{n}^{2}, D_{n}^{2}$ and $E_{6}^{2}$ [2, pp. 1746-1747], the diagrams of $\sigma$ and $\sigma \theta$ always appear in nonequivalent pairs (they coincide on $\mathrm{D}_{\mathfrak{k}}$, but the black vertex is circled in one diagram and not circled in the other).

Theorem 1.7 assumes that the diagram automorphism $d$ is trivial. The situation is more complicated if $d$ is nontrivial. Suppose that $\operatorname{ord}(d)=2, d$ preserves $\mathrm{D} \subset \mathrm{D}^{1}$, and $\Pi_{\alpha \in \mathcal{O}} c_{\alpha}=1$ for every $d$-orbit $\mathcal{O}$. Then we can form the quotient diagram whose vertices are $d$-orbits, whose edges follow the root lengths

$$
\text { special } d \text {-orbit < nonspecial } d \text {-orbit }<\text { vertex fixed by } d \text {, }
$$

and use the above method to find $\mathfrak{g}_{0}^{\sigma}$. But for more general markings, we do not yet know how they reveal $\mathfrak{g}_{0}^{\sigma}$. 


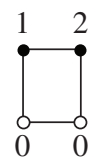

(a)

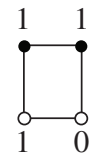

(b)

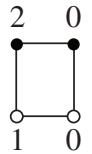

(c)

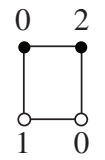

(d)

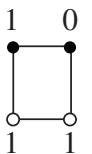

(e)

FiguRE 5

\section{EXAMPLES}

In this section, we give three examples to illustrate the ideas discussed before.

Example 9.1. Automorphisms on $\mathfrak{s u}(3,1)$ of order 3 .

We shall classify $\operatorname{aut}_{3}(\mathfrak{s u}(3,1))$ up to conjugation by aut $(\mathfrak{s u}(3,1))$ and study their fixed point sets. By Theorem 1.3. the nontrivial diagram automorphism on the painted diagram of $\mathfrak{s u}(3,1)$ leads to even order automorphisms, so it is irrelevant in $\operatorname{aut}_{3}(\mathfrak{s u}(3,1))$ here. The marking $c$ assigns values of $\left(\exp \frac{2 \pi i}{3}\right)^{a}$ to the vertices, where $a \in \mathbb{Z}_{3}$. To simplify notation, we identify $a \in \mathbb{Z}_{3}$ with $\left(\exp \frac{2 \pi i}{3}\right)^{a} \in \mathbb{C}$ and work with $\mathbb{Z}_{3}$. So $c_{\alpha} \in \mathbb{Z}_{3}=\{0,1,2\}$ in Figure 5 . We claim that Figure 5 classifies $\operatorname{aut}_{3}(\mathfrak{s u}(3,1))$.

We now construct admissible markings $c$, namely $\sum_{\mathrm{P}^{1}} c_{\alpha}=0 \in \mathbb{Z}_{3}$. Let $\alpha, \beta$ be the left and right white vertices of $\mathrm{P}^{1}$ as they appear in Figure 5. Given $\sigma \in$ $\operatorname{aut}_{3}(\mathfrak{s u}(3,1))$, by Corollary 2.4, $\sigma$ is an extension from $\mathfrak{k}_{0}=\mathfrak{s}(\mathfrak{u}(3) \oplus \mathfrak{u}(1))$. So we start with the following markings $\left(c_{\alpha}, c_{\beta}\right)$ on $\mathrm{D}_{\mathfrak{k}}$, where $c_{\alpha}, c_{\beta} \in \mathbb{Z}_{3}$ :

$$
A=(0,0), B=(1,0), C=(1,1), D=(2,0), E=(2,1), F=(2,2) .
$$

We intend to classify $\operatorname{aut}_{3}(\mathfrak{s u}(3,1))$ only up to conjugation by aut $(\mathfrak{s u}(3,1))$ and not $\operatorname{int}(\mathfrak{s u}(3,1))$. So by Theorem 1.4, we omit markings which differ by diagram symmetry. For example we omit $(0,1)$ because we have listed $(1,0)$. We can further shorten the list (9.1) by the reflection algorithms of Theorem 1.4. For the additive group $\mathbb{Z}_{3}$, (5.2) gives $\left(F_{\alpha} c\right)_{\beta}=c_{\alpha}+c_{\beta}$ if $\alpha, \beta$ are adjacent vertices, and $\left(F_{\alpha} c\right)_{\alpha}=-c_{\alpha}$. For instance $F_{\alpha} B=F_{\alpha}(1,0)=(-1,1+0)=(2,1)$, so any extension of $E$ is conjugate to an extension of $B$. Also, $F_{\beta} E=F_{\beta}(2,1)=(1+2,-1)=(0,2)$, so any extension of $E$ is conjugate to an extension of $D$. Hence we keep $B$ and omit $D$ and $E$ from (9.1). Similarly, since $F_{\alpha} C=F_{\alpha}(1,1)=(-1,1+1)=(2,2)$, we keep $C$ and omit $F$. Therefore, we only need extensions of $A, B$ and $C$ to admissible markings. Admissible extensions of $A$ and $B$ lead to Figures 5(a)-(d). The marking $C$ has two admissible extensions, $C_{1}=\left(\begin{array}{ll}1 & 0 \\ 1 & 1\end{array}\right)$ and $C_{2}=\left(\begin{array}{ll}2 & 2 \\ 1 & 1\end{array}\right)$. Since $F_{\alpha} \cdot F_{\beta}$ transforms $C_{2}$ to $C_{1}$, we list only $C_{1}$ as Figure $5(\mathrm{e})$. We conclude that Figure 5 represents all the conjugate classes of $\operatorname{aut}_{3}(\mathfrak{s u}(3,1))$.

Next we use Theorem 1.7 to compute the fixed point sets $\mathfrak{g}_{0}^{\sigma}$. Each marking of Figure 5 is already $\mathfrak{g}$-standard in the sense of (8.2), so we can use it to compute $\mathfrak{g}^{\sigma}$ directly.

We start with Figure 5(a). Its zero entries form an $A_{2}$-diagram, and it has two nonzero entries. By Theorem 1.7(a),

$$
\mathfrak{g}^{\sigma}=A_{2}+\mathbb{C} .
$$




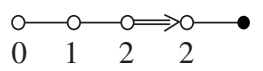

(a)

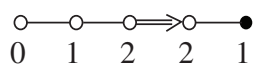

(b)

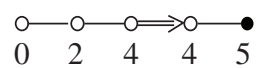

(c)

FiguRE 6

All white vertices are assigned zero, so $\sigma=1$ on $\mathfrak{k}_{0}$. Hence

$$
\mathfrak{k}^{\sigma}=A_{2}+\mathbb{C} .
$$

By (9.2) and (9.3), $\mathfrak{g}_{0}^{\sigma}=\mathfrak{s u}(3)+\mathfrak{s o}(2)$.

Next consider Figure $5(\mathrm{~b})$. Its zero entries form an $A_{1}$-diagram, and it has three nonzero entries. By Theorem 1.7(a),

$$
\mathfrak{g}^{\sigma}=A_{1}+\mathbb{C}^{2} .
$$

The two white vertices are assigned 1 and 0 . Add a vertex $\varphi$ to form $\left(D_{\mathfrak{k}}\right)^{1}=A_{2}^{1}$, and let $c_{\varphi}=2$ so that $\sum_{\left(\mathrm{D}_{\mathfrak{k}}\right)^{1}} b_{\alpha} c_{\alpha}=\sum_{\left(\mathrm{D}_{\mathfrak{k}}\right)^{1}} c_{\alpha}=0 \in \mathbb{Z}_{3}$. Here $\left\{b_{\alpha}=1\right\}$ are the labels of $\left(D_{\mathfrak{k}}\right)^{1}$. Since $\left(D_{\mathfrak{k}}\right)^{1}$ has one zero entry and two nonzero entries, by Theorem 1.7(b),

$$
\mathfrak{k}^{\sigma}=A_{1}+\mathbb{C}^{2} .
$$

By (9.4) and (9.5), $\mathfrak{g}_{0}^{\sigma}=\mathfrak{s u}(2)+\mathfrak{s o}(2)+\mathfrak{s o}(2)$.

Now consider Figure $5(\mathrm{c})$. Its zero entries form an $A_{2}$-diagram, and it has two nonzero entries. By Theorem 1.7(a),

$$
\mathfrak{g}^{\sigma}=A_{2}+\mathbb{C} .
$$

The two white vertices are assigned 1 and 0 , which is the same as Figure $5(\mathrm{~b})$. So we again have (9.5). By (9.5) and (9.6), $\mathfrak{g}_{0}^{\sigma}=\mathfrak{s u}(2,1)+\mathfrak{s o}(2)$.

One may similarly check that Figure $5(\mathrm{~d})$ gives

$$
\mathfrak{g}^{\sigma}=A_{1}+A_{1}+\mathbb{C}, \mathfrak{k}^{\sigma}=A_{1}+\mathbb{C}^{2} \Longrightarrow \mathfrak{g}_{0}^{\sigma}=\mathfrak{s u}(2)+\mathfrak{s u}(1,1)+\mathfrak{s o}(2)
$$

and Figure 5(e) gives

$$
\mathfrak{g}^{\sigma}=A_{1}+\mathbb{C}^{2}, \mathfrak{k}^{\sigma}=\mathbb{C}^{3} \Longrightarrow \mathfrak{g}_{0}^{\sigma}=\mathfrak{s u}(1,1)+\mathfrak{s o}(2)+\mathfrak{s o}(2) .
$$

Example 9.2. Extensions of $\sigma \in \operatorname{aut}_{3}(\mathfrak{s o}(9))$ to aut $\left(\mathfrak{f}_{4(-20)}\right)$.

Consider $\mathfrak{g}_{0}=\mathfrak{f}_{4(-20)}$, with $\mathfrak{k}_{0}=\mathfrak{s o}(9)$. This example demonstrates the extensions of some $\sigma \in$ aut $_{3}\left(\mathfrak{k}_{0}\right)$ to $\operatorname{aut}_{m}\left(\mathfrak{g}_{0}\right)$ and studies their fixed point sets. By Corollary 1.6, $m \in\{3,6\}$. The painted diagram of $\mathfrak{f}_{4(-20)}$ appears in Figure 2 of Section 3 .

Let $\sigma \in$ aut $_{3}\left(\mathfrak{k}_{0}\right)$ be represented by the marking $c: \mathrm{D}_{\mathfrak{k}} \longrightarrow \mathbb{Z}_{3}$ in Figure 6(a), where we again identify $a \in \mathbb{Z}_{3}$ with $\left(\exp \frac{2 \pi i}{3}\right)^{a} \in \mathbb{C}$.

The labels $a_{\alpha}$ of $F_{4}^{1}$ are 1,2,3,4,2 from left to right in Figure 6. By (1.7), Figure $6\left(\right.$ a) gives $w=\Pi_{\mathrm{D}_{\mathfrak{k}}} c_{\alpha}^{a_{\alpha}}=\left(\exp \frac{2 \pi i}{3}\right)^{0 \cdot 1+1 \cdot 2+2 \cdot 3+2 \cdot 4}=\left(\exp \frac{2 \pi i}{3}\right)^{16}=\exp \frac{2 \pi i}{3}$. Therefore, by Corollary 1.6, $\sigma$ extends to aut $\left(\mathfrak{g}_{0}\right)$ of order

$$
\operatorname{lcm}\left\{n, \operatorname{ord}\left( \pm w^{\frac{1}{2}}\right)\right\}=\operatorname{lcm}\left\{3, \operatorname{ord}\left( \pm(\exp 2 \pi i / 3)^{\frac{1}{2}}\right)\right\} .
$$

The positive sign in (9.7) gives $\operatorname{lcm}\left\{3, \operatorname{ord}\left(\exp \frac{\pi i}{3}\right)\right\}=\operatorname{lcm}\{3,6\}=6$. The negative sign in (9.7) gives $\operatorname{lcm}\left\{3, \operatorname{ord}\left(\exp \frac{4 \pi i}{3}\right)\right\}=\operatorname{lcm}\{3,3\}=3$. Hence $\sigma$ has one extension to $\operatorname{aut}_{3}\left(\mathfrak{g}_{0}\right)$ and one extension to $\operatorname{aut}_{6}\left(\mathfrak{g}_{0}\right)$. 


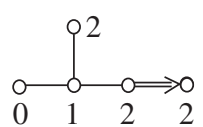

(a)

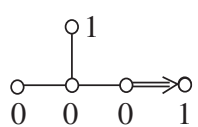

(b)

FiguRE 7

We write $a \in \mathbb{Z}_{3}$ for $\left(\exp \frac{2 \pi i}{3}\right)^{a} \in \mathbb{C}$ in Figure $6(\mathrm{~b})$ and write $a \in \mathbb{Z}_{6}$ for $\left(\exp \frac{2 \pi i}{6}\right)^{a} \in \mathbb{C}$ in Figure 6(c). Then Figure 6(b), (c) indeed represent the extensions of $\sigma$ to aut ${ }_{3}\left(\mathfrak{g}_{0}\right)$ and aut $_{6}\left(\mathfrak{g}_{0}\right)$, respectively. In Figure $6(\mathrm{~b})$,

$$
\Pi_{\mathrm{P}^{1}} c_{\alpha}^{a_{\alpha}}=\left(\exp \frac{2 \pi i}{3}\right)^{0 \cdot 1+1 \cdot 2+2 \cdot 3+2 \cdot 4+1 \cdot 2}=\left(\exp \frac{2 \pi i}{3}\right)^{18}=1,
$$

so it is an admissible marking. Similarly, in Figure 6(c),

$$
\Pi_{\mathrm{P}^{1}} c_{\alpha}^{a_{\alpha}}=\left(\exp \frac{2 \pi i}{6}\right)^{0 \cdot 1+2 \cdot 2+4 \cdot 3+4 \cdot 4+5 \cdot 2}=\left(\exp \frac{2 \pi i}{6}\right)^{42}=1,
$$

so it is also an admissible marking. One can further check that $1 \in \mathbb{Z}_{3}$ and $5 \in \mathbb{Z}_{6}$ on the black vertex are the only admissible extensions of Figure 6(a).

We next apply Theorem 1.7 to find the fixed points $\mathfrak{g}_{0}^{\sigma}$ for Figures $6(\mathrm{~b}),(\mathrm{c})$. We start with Figure 6(b). We again work with $a \in \mathbb{Z}_{3}$. By (8.2), Figure 6(b) is not $\mathfrak{g}$-standard, so we apply reflection algorithms (8.1) to obtain a $\mathfrak{g}$-standard by

$$
\begin{aligned}
& (0,1,2, \underline{2}, 1) \rightarrow(0, \underline{1}, 0,1,0) \rightarrow(1,2, \underline{1}, 1,0) \\
& \longrightarrow(1,0,2, \underline{2}, 0) \rightarrow(1,0,0,1, \underline{2}) \rightarrow(1,0,0,0,1) .
\end{aligned}
$$

Each underlined entry in (9.8) indicates the vertex $\alpha$ for the reflection algorithm $F_{\alpha}$. For example, the first step of (9.8) applies (5.2) and transforms $(0,1,2,2,1)$ to $(0,1,2(2)+2,-2,2+1)=(0,1,0,1,0)$. Similarly, the second step of (9.8) transforms $(0,1,0,1,0)$ to $(1+0,-1,1+0,1,0)=(1,2,1,1,0)$. The last marking of (9.8) is $\mathfrak{g}$-standard because it satisfies (8.2). Applying Theorem 1.7(a) to the last marking of (9.8), we get

$$
\mathfrak{g}^{\sigma}=B_{3}+\mathbb{C}
$$

Next we compute $\mathfrak{k}^{\sigma}$ of Figure $6(\mathrm{~b})$. Add the lowest root $\varphi$ of $\mathfrak{k}$ to the white vertices $\mathrm{D}_{\mathfrak{k}}$ to obtain $\left(\mathrm{D}_{\mathfrak{k}}\right)^{1}=B_{4}^{1}$, and let $\left\{b_{\alpha}\right\}$ be its labels. Let $c_{\varphi}=2$ so that $\Pi_{\left(\mathrm{D}_{\mathfrak{k}}\right)^{1}} c_{\alpha}^{b_{\alpha}}=\left(\exp \frac{2 \pi i}{3}\right)^{0 \cdot 1+1 \cdot 2+2 \cdot 2+2 \cdot 2+2 \cdot 1}=1$. This leads to Figure $7(\mathrm{a})$.

By (8.5), Figure $7(\mathrm{a})$ is not $\mathfrak{k}$-standard. Apply reflection algorithms to Figure 7(a), and obtain Figure 7(b) by

$$
\left(\begin{array}{cccc} 
& 2 & & \\
0 & 1 & 2 & \underline{2}
\end{array}\right) \rightarrow\left(\begin{array}{cccc} 
& \underline{2} & & \\
0 & 1 & 0 & 1
\end{array}\right) \rightarrow\left(\begin{array}{cccc} 
& 1 & & \\
0 & 0 & 0 & 1
\end{array}\right)
$$

Figure 7(b) is k-standard. Applying Theorem 1.7(b) to Figure 7(b), we get

$$
\mathfrak{k}^{\sigma}=A_{3}+\mathbb{C} .
$$

By (9.9) and (9.10), we conclude that $\mathfrak{g}_{0}^{\sigma}=\mathfrak{s o}(6,1)+\mathfrak{s o}(2)$ in Figure 6(b).

Next we find $\mathfrak{g}_{0}^{\sigma}$ for Figure $6(\mathrm{c})$. We work with $\mathbb{Z}_{6}$ below. By (8.2), Figure 6(c) is not $\mathfrak{g}$-standard, so we apply reflection algorithms (8.1) and get

$$
\begin{aligned}
& (0,2,4,4, \underline{5}) \rightarrow(0,2,4, \underline{3}, 1) \rightarrow(0,2,4,3, \underline{4}) \\
& \rightarrow(0,2, \underline{4}, 1,2) \rightarrow(0,0,2, \underline{5}, 2) \rightarrow(0,0,0,1,1) .
\end{aligned}
$$




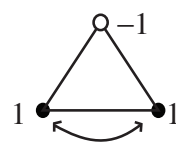

(a)

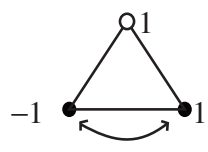

(b)

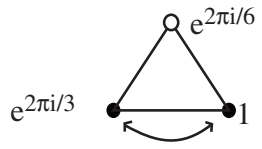

(c)

FIGURE 8

The last marking of (9.11) is $\mathfrak{g}$-standard because it satisfies (8.2). Applying Theorem 1.7 (a) to the last marking of (9.11), we get

$$
\mathfrak{g}^{\sigma}=A_{3}+\mathbb{C} .
$$

By (9.10) and (9.12), we conclude that $\mathfrak{g}_{0}^{\sigma}=\mathfrak{s u}(4)+\mathfrak{s o}(2)$ in Figure 6(c).

It can be difficult to judge equivalent markings or find standard markings, such as finding the reflection algorithms in (9.8) and (9.11) to obtain $\mathfrak{g}$-standard markings. A systematic treatment is given in [1 for $\mathfrak{g}$-involutions, but the combinatorial method for higher order automorphisms remains unclear.

Example 9.3. Outer automorphisms on $\mathfrak{s u}(2,1)$ of order $\leq 6$.

In this example, we classify outer automorphisms on $\mathfrak{s u}(2,1)$ of order $\leq 6$, up to conjugation by aut $(\mathfrak{s u}(2,1))$. The painted diagram $\mathrm{P}^{1}$ of $\mathfrak{s u}(2,1)$ has one white vertex $\alpha$ and two black vertices $\gamma, \delta$. If $\sigma \in \operatorname{out}_{m}(\mathfrak{s u}(2,1))$ is represented by a marking $(c, d)$ on $\mathrm{P}^{1}$, then $d$ interchanges $\gamma$ and $\delta$. Since $\{\gamma, \delta\}$ is a special $d$-orbit, condition (1.5) for an admissible marking is

$$
-c_{\alpha} c_{\gamma} c_{\delta}=1
$$

If it is admissible, then by Theorem 1.3 its order is

$$
m=\operatorname{lcm}\left\{\operatorname{ord}\left(c_{\alpha}\right), 2 \operatorname{ord}\left(c_{\gamma} c_{\delta}\right)\right\} .
$$

So $m$ has to be even, and we consider $m=2,4,6$. We claim that they are all given by Figure 8 . The numbers in Figure 8 belong to $U$.

We now solve for $c_{\alpha}$ and $c_{\gamma} c_{\delta}$ in (9.13) and (9.14). By Theorem 1.4 and (5.9) (b), we need not solve $c_{\gamma}$ and $c_{\delta}$ separately because they all result in equivalent markings.

For $m=2$, the only solution of (9.13) and (9.14) is $c_{\alpha}=-1$ and $c_{\gamma} c_{\delta}=1$ so that $-c_{\alpha} c_{\gamma} c_{\delta}=-(-1) 1=1$ and

$$
\operatorname{lcm}\left\{\operatorname{ord}\left(c_{\alpha}\right), 2 \operatorname{ord}\left(c_{\gamma} c_{\delta}\right)\right\}=\operatorname{lcm}\{\operatorname{ord}(-1), 2 \operatorname{ord}(1)\}=\operatorname{lcm}\{2,2\}=2 .
$$

Let $c_{\gamma}=c_{\delta}=1$, and we get Figure $8(\mathrm{a})$.

For $m=4$, the only solution of (9.13) and (9.14) is $c_{\alpha}=1$ and $c_{\gamma} c_{\delta}=-1$ so that $-c_{\alpha} c_{\gamma} c_{\delta}=-(1)(-1)=1$ and

$$
\operatorname{lcm}\left\{\operatorname{ord}\left(c_{\alpha}\right), 2 \operatorname{ord}\left(c_{\gamma} c_{\delta}\right)\right\}=\operatorname{lcm}\{\operatorname{ord}(1), 2 \operatorname{ord}(-1)\}=\operatorname{lcm}\{1,4\}=4 .
$$

We choose $c_{\gamma}=-1, c_{\delta}=1$, and get Figure $8(\mathrm{~b})$.

For $m=6$, there are two solutions of (9.13) and (9.14) given by

$$
\text { (a) }\left(c_{\alpha}, c_{\gamma} c_{\delta}\right)=\left(e^{2 \pi i / 6}, e^{2 \pi i / 3}\right), \quad \text { (b) }\left(c_{\alpha}^{\prime}, c_{\gamma}^{\prime} c_{\delta}^{\prime}\right)=\left(e^{10 \pi i / 6}, e^{4 \pi i / 3}\right) \text {. }
$$

We verify that (9.15) (a) satisfies $-c_{\alpha} c_{\gamma} c_{\delta}=-e^{2 \pi i / 6} e^{2 \pi i / 3}=1$ and

$$
\operatorname{lcm}\left\{\operatorname{ord}\left(c_{\alpha}\right), 2 \operatorname{ord}\left(c_{\gamma} c_{\delta}\right)\right\}=\operatorname{lcm}\left\{\operatorname{ord}\left(e^{2 \pi i / 6}\right), 2 \operatorname{ord}\left(e^{2 \pi i / 3}\right)\right\}=\operatorname{lcm}\{6,6\}=6 .
$$




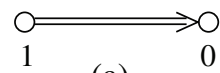

(a)

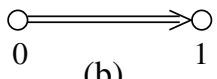

(b)

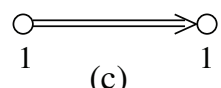

(c)

Figure 9

From (9.15) (a), choose $c_{\gamma}=e^{2 \pi i / 3}$ and $c_{\delta}=1$. This gives Figure $8(\mathrm{c})$. The values in (9.15) (b) are multiplicative inverses of (9.15) (a), so (9.15) (a), (b) represent automorphisms which are inverses of each other. Since $F_{\alpha} c_{\alpha}=c_{\alpha}^{-1}=c_{\alpha}^{\prime}$, any extension of a $\mathfrak{k}$-automorphism represented by $c_{\alpha}^{\prime}$ is conjugate by aut( $\left.\mathfrak{g}\right)$ to some extension of a $\mathfrak{k}$-automorphism represented by $c_{\alpha}$. Hence (9.15) (b) is redundant. We may also directly check that (9.15) (a), (b) are equivalent by

$$
\begin{array}{rlrl}
\left(c_{\alpha}, c_{\gamma}, c_{\delta}\right) & =\left(e^{2 \pi i / 6}, e^{2 \pi i / 3}, 1\right) & \\
& \rightarrow\left(e^{-2 \pi i / 6}, e^{2 \pi i / 6} e^{2 \pi i / 3}, e^{2 \pi i / 6}\right) & \text { by } F_{\alpha} \text { in (5.9) (a) } \\
& =\left(e^{10 \pi i / 6},-1, e^{2 \pi i / 6}\right) & & \\
& \rightarrow\left(e^{10 \pi i / 6}, e^{4 \pi i / 3}, 1\right) & & \text { by (15.9) (b) } \\
& =\left(c_{\alpha}^{\prime}, c_{\gamma}^{\prime}, c_{\delta}^{\prime}\right) . &
\end{array}
$$

We conclude that Figure 8 classifies all out $_{m}(\mathfrak{s u}(2,1))$ for $m \leq 6$, up to conjugation by $\operatorname{aut}(\mathfrak{s u}(2,1))$.

If $\sigma \in \operatorname{out}_{m}(\mathfrak{s u}(2,1))$, its complexifies to out $m(\mathfrak{s l}(3, \mathbb{C}))$. Let $A_{2}^{2}$ be the affine Dynkin diagram resulting from the involution on $A_{2}$. By Theorem 2.1(a) and (4.4)(b), $\sigma \in \operatorname{out}_{m}(\mathfrak{s l}(3, \mathbb{C}))$ can be represented by nonnegative integers $\left\{n_{\alpha}\right\}_{A_{2}^{2}}$ without nontrivial common factor such that $2 \sum_{A_{2}^{2}} a_{\alpha} n_{\alpha}=m$. The labels $a_{\alpha}$ of $A_{2}^{2}$ are 1,2 from left to right as they appear in Figure 9. We check that Figure 9 provides all solutions of $\left\{n_{\alpha}\right\}_{A_{2}^{2}}$ for $m \leq 6$.

In Figure 9(a), $2 \sum_{A_{2}^{2}} a_{\alpha} n_{\alpha}=2(1 \cdot 1+2 \cdot 0)=2$, so it represents $\operatorname{out}_{2}(\mathfrak{s l}(3, \mathbb{C}))$. In Figure 9(b), $2 \sum_{A_{2}^{2}} a_{\alpha} n_{\alpha}=2(1 \cdot 0+2 \cdot 1)=4$, so it represents out $4(\mathfrak{s l}(3, \mathbb{C}))$. In Figure 9(c), $2 \sum_{A_{2}^{2}} a_{\alpha} n_{\alpha}=2(1 \cdot 1+2 \cdot 1)=6$, so it represents out $6(\mathfrak{s l}(3, \mathbb{C}))$. Hence the complexifications of Figure 8(a), (b), (c) lead to Figure 9(a), (b), (c) respectively. Figure 8 is more informative than Figure 9, as it takes the real form $\mathfrak{s u}(2,1)$ into account.

\section{Appendix: InNer isomorphic REAL FORMS}

In Section 3, Figures 1, 2 and 3 provide all the real forms up to isomorphism. Given isomorphic real forms $\mathfrak{g}_{0}, \mathfrak{g}_{1} \subset \mathfrak{g}$, if there exists an isomorphism which extends to $\operatorname{int}(\mathfrak{g})$, we say that $\mathfrak{g}_{0}$ and $\mathfrak{g}_{1}$ are inner isomorphic. We now show that the additional inner isomorphic classes are given by Figure 4 of Section 3, so that Figures 1, 2, 3 and 4 provide a complete list of inner isomorphic classes of real forms.

Let $\tilde{\mathfrak{h}}$ be a Cartan subalgebra of $\mathfrak{g}$ (to be consistent with earlier notation, we reserve $\mathfrak{h}$ for the Cartan subalgebra of $\mathfrak{k})$. Let $W \subset \operatorname{aut}(\Delta(\mathfrak{g}, \tilde{\mathfrak{h}}))$ be the Weyl group of $\mathfrak{g}$.

Proposition 10.1 ([8, Ch. VII, Thm. 7.8]). Suppose that $\phi \in \operatorname{aut}(\mathfrak{g})$ preserves $\tilde{\mathfrak{h}}$. Define $d \in \operatorname{aut}(\Delta(\mathfrak{g}, \tilde{\mathfrak{h}}))$ by $\phi \mathfrak{g}_{\alpha}=\mathfrak{g}_{d \alpha}$. Then $\phi \in \operatorname{int}(\mathfrak{g})$ if and only if $d \in W$.

We first discuss affine Dynkin diagrams of type $\mathrm{D}^{1}$. Let $\mathcal{P}$ be the family of all paintings (assignments of white or black) on the vertices of $\mathrm{D}^{1}$. Let $c \in \mathcal{P}$. Thus 
$c_{\alpha}$ is white or black for all $\alpha \in \mathrm{D}^{1}$. If $c_{\alpha}$ is black, define another painting $F_{\alpha} c \in \mathcal{P}$ by:

$$
\begin{aligned}
& c_{\beta} \text { and }\left(F_{\alpha} c\right)_{\beta} \text { have opposite colors } \Longleftrightarrow \\
& \alpha, \beta \text { are adjacent, and either }|\alpha| \geq|\beta| \text { or } \beta \equiv \equiv>\alpha .
\end{aligned}
$$

Here "三三>" is the notation from Proposition 2.7. Formula (10.1) is the same as [1. (2.1)]. We use it to define an equivalence relation on $\mathcal{P}$ by $c_{0} \sim c_{1}$ if and only if $c_{1}=F_{\alpha_{n}} \cdot \ldots \cdot F_{\alpha_{1}} c_{0}$ for some $F_{\alpha_{i}}$. This is similar to Definition 5.3 for the markings.

Recall that, by Corollary 2.2 if a painting on $\mathrm{D}^{1}$ satisfies $\sum_{\text {black }} a_{\alpha}=2$, then it represents a real form of $\mathfrak{g}$ in the sense of Definition 1.1. As explained in (2.2), for $\mathrm{D}^{1}, \mathfrak{g}$ and $\mathfrak{k}$ have the common Cartan subalgebra $\tilde{\mathfrak{h}}=\mathfrak{h}$. Let $\Delta=\Delta(\mathfrak{g}, \mathfrak{h})$.

Proposition 10.2. Let $c_{0}, c_{1} \in \mathcal{P}$ represent real forms $\mathfrak{g}_{0}, \mathfrak{g}_{1} \subset \mathfrak{g}$, respectively. Then $c_{0} \sim c_{1}$ if and only if $\mathfrak{g}_{0}$ and $\mathfrak{g}_{1}$ are inner isomorphic.

Proof. Suppose that $c \in \mathcal{P}$ represents $\mathfrak{g}_{0}$ by letting the vertices of $\mathrm{D}^{1}$ correspond to $\Pi$. We may also write $c_{\alpha}=1$ if $\alpha$ is white and $c_{\alpha}=-1$ if $\alpha$ is black. Pick a black vertex $\alpha$, namely $c_{\alpha}=-1$. Let $r_{\alpha}$ be the reflection on $\Delta$ defined by $\alpha$. Similar to the arguments of Propositions 2.7 and [5.1, the painting with respect to $r_{\alpha} \Pi$ is $F_{\alpha} c$, where $F_{\alpha}$ is given by (5.2). In (5.2), $c_{\alpha}^{s} c_{\beta}=-c_{\beta}$ if and only if $s$ is odd, so $\left(F_{\alpha} c\right)_{\beta}=-c_{\beta}$ if and only if $\alpha, \beta$ are adjacent, and either $|\alpha| \geq|\beta|$ or $\beta \equiv \equiv>\alpha$. This is the condition in (10.1).

For $i=0,1$, let $c_{i} \in \mathcal{P}$ represent $\mathfrak{g}_{i}$ with respect to the simple system $\Pi_{i}$ of $\Delta$. Let $\phi \in \operatorname{aut}(\mathfrak{g})$ satisfy $\phi\left(\mathfrak{g}_{0}\right)=\mathfrak{g}_{1}$ and preserve $\mathfrak{h}$. Define $d \in \operatorname{aut}(\Delta)$ by $\phi \mathfrak{g}_{\alpha}=\mathfrak{g}_{d \alpha}$, and $d \Pi_{0}=\Pi_{1}$. By Proposition 10.1 $\phi \in \operatorname{int}(\mathfrak{g})$ if and only if $d \in W$. Here $W$ is generated by the simple reflections $\left\{r_{\alpha}\right\}_{\alpha}$, and the effect of $r_{\alpha}$ on the painting is given by (10.1). Hence there exists $\phi \in \operatorname{int}(\mathfrak{g})$ satisfying $\phi\left(\mathfrak{g}_{0}\right)=\mathfrak{g}_{1}$ if and only if $c_{0} \sim c_{1}$.

We look for all diagram symmetries of the painted diagrams in Figures 1 and 2. For example, in Figure 1 all diagram symmetries of $\mathfrak{s u}(p, q)$ are obtained by shifting the pair of black vertices along the loop $A_{n}^{1}$. We then check whether the resulting painted diagrams are equivalent to the original ones. By Proposition 10.2. this determines whether the corresponding real forms are inner isomorphic.

The painted diagrams on $\mathrm{D}^{1}$ with nontrivial diagram symmetries are as follows:

Figure 1: $\mathfrak{s u}(p, q), \mathfrak{s o}(2,2 n-2), \mathfrak{s o}^{*}(2 n), \mathfrak{e}_{6(-14)}$.

Figure 2: $\mathfrak{s p}(p, q), \mathfrak{s o}(2 p, 2 q), \mathfrak{e}_{6(2)}, \mathfrak{e}_{7(-5)}$.

If the vertices of $\mathrm{D}^{1}$ are labeled by $0,1, \ldots, n$, let $\left(i_{1}, \ldots, i_{k}\right)$ denote the painting where vertices $i_{1}, \ldots, i_{k} \in\{0, \ldots, n\}$ are black, while the remaining vertices are white. For example, if the vertices of $A_{n}^{1}$ are labeled $0, \ldots, n$ consecutively, then the painted diagram for $\mathfrak{s u}(p, q)$ is $(0, p)$, and (10.1) gives $F_{p}(0, p)=(0, p-1, p, p+1)$. We now check the above cases one by one.

$$
\mathfrak{s u}(p, q)
$$

Label the vertices of $A_{n}^{1}$ by $\{0,1, \ldots, n\}$ consecutively. The painted diagram for $\mathfrak{s u}(p, q)$ is $(0, p)$. We have $F_{1} \cdot \ldots \cdot F_{p}(0, p)=F_{1} \cdot \ldots \cdot F_{p-1}(0, p-1, p, p+1)=\ldots=$ $F_{1}(0,1,2, p+1)=(1, p+1)$ (a slight modification if $\left.p=n\right)$. Hence $(0, p) \sim(1, p+1)$. Continue this argument inductively; we see that all diagram symmetries of $(0, p)$ are equivalent. 
The next two cases deal with painted diagrams on $D_{n}^{1}$, where $n \geq 5$. We shall discuss $D_{4}^{1}$ separately later, due to its extra symmetry.

$$
\mathfrak{s o}(2,2 n-2), n \geq 5
$$

Label the vertices of $D_{n}^{1}$ by

$$
\left(\begin{array}{ccccc}
0 & & & & n-1 \\
& 2 & \ldots & n-2 & \\
1 & & & & n
\end{array}\right) .
$$

The painted diagram in Figure 1 is $(0,1)$, and its nontrivial diagram symmetry is $(n-1, n)$. Apply $F_{0}, F_{2}, F_{3}, \ldots, F_{n-1}$ to $(0,1)$, and we obtain $(n-1, n)$. So $(0,1) \sim(n-1, n)$.

$$
\mathfrak{s o}^{*}(2 n), n \geq 5
$$

This appears on $D_{n}^{1}$. Use the labeling (10.2), and the painted diagram in Figure 1 is $(0, n-1)$. Its three nontrivial diagram symmetries are $(1, n),(1, n-1),(0, n)$. For $i=0,1$, we apply $F_{i}, F_{2}, F_{3}, \ldots, F_{n-2}, F_{n}$ to $(i, n-1)$. This leads to

$$
(0, n-1) \sim(1, n),(1, n-1) \sim(0, n) .
$$

In (10.3), it remains to consider whether $(0, n) \sim(1, n)$. We shall show that

$$
n \text { is odd } \Longleftrightarrow(0, n) \sim(1, n) .
$$

Suppose that $n$ is odd. We claim that for all $p=4, \ldots, n-2$,

$$
\text { (a) }(0, p, n) \sim(1, p-1, n) \sim(0, p-2, n) \text {, (b) }(0, n) \sim(1, n-3, n) \text {. }
$$

We get (10.5) (a) by applying $F_{0}, F_{2}, F_{3}, \ldots, F_{p-1}$ to $(0, p, n)$, followed by $F_{1}, F_{2}, \ldots$, $F_{p-2}$ to $(1, p-1, n)$. We also get (10.5) (b) by applying $F_{0}, F_{2}, F_{3}, \ldots, F_{n-3}, F_{n}$ to $(0, n)$. This proves (10.5), as claimed.

By (10.5) (a), (b), we get $(0, n) \sim(1, n-3, n) \sim(1, n-5, n) \sim \ldots \sim(1,2, n)$ because $n$ is odd. Finally, $(1,2, n) \sim(1, n)$. This proves the " $\Longrightarrow$ " part of (10.4).

For the converse part of (10.4), suppose that $n$ is even. Assume that $F_{i_{r}} \cdot \ldots$. $F_{i_{1}}(0, n)=(1, n)$. The colors of vertices 0,1 imply that 2 appears an odd number of times in $\left\{i_{1}, \ldots, i_{r}\right\}$ because $F_{2}$ is the only operation that changes the colors of vertices 0 and 1 . Since vertex 3 remains white, it further implies that 4 also appears an odd number of times in $\left\{i_{1}, \ldots, i_{r}\right\}$ because $F_{2}$ and $F_{4}$ are the only operations that change the color of vertex 3 . Similarly, $6,8, \ldots, n-2$ all appear an odd number of times in $\left\{i_{1}, \ldots, i_{r}\right\}$. The last condition is a contradiction because the colors of vertices $n-1, n$ have not changed. This completes the proof of (10.4). By (10.3) and (10.4), we obtain the painted diagrams in Figure 4.

$$
\mathfrak{s o}(4,4)
$$

Label the vertices of $D_{4}^{1}$ by $0, \ldots, 4$, where 0 is the central vertex. There are six painted diagrams for $\mathfrak{s o}(4,4)$, and direct computation shows that

$$
(1,2) \sim(3,4),(1,3) \sim(2,4),(1,4) \sim(2,3) .
$$

For example $F_{3} \cdot F_{0} \cdot F_{1}(1,2)=(3,4)$, and so on. Vertices $1,2,3,4$ may change colors only by $F_{0}$, and $F_{0}$ changes the colors of vertices $1,2,3,4$ simultaneously. This implies that the three partitions in (10.6) are mutually not equivalent. For instance, any painted diagram which is equivalent to $(1,2)$ needs to have the same 


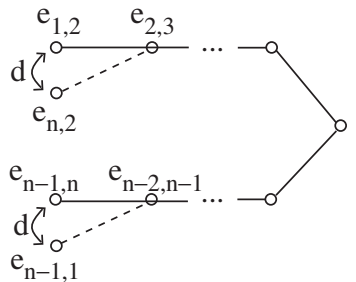

(a)

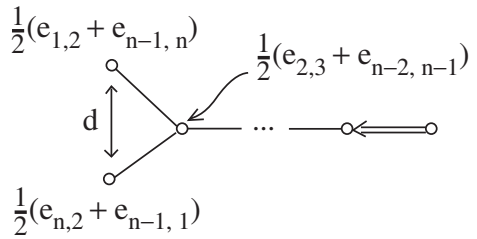

(b)

FiguRe 10

color for vertices 1 and 2 , so $(1,2)$ cannot be equivalent to $(1,3)$, and so on. Hence there are altogether three equivalence classes, and we list them in Figure 4.

Label the vertices by $\left(\begin{array}{rrrrr} & & \mathfrak{e}_{6(-14)} & & \\ & & 1 & & \\ 2 & 3 & 4 & 5 & 6\end{array}\right)$. The painted diagram in Figure 1 is $(2,6)$. By applying $F_{2}, F_{3}, F_{4}, F_{1}, F_{0}, F_{6}$, we get $(2,6) \sim(0,6)$. Similar arguments give $(2,6) \sim(0,2)$.

$$
\mathfrak{s p}(p, q), \mathfrak{s o}(2 p, 2 q) \text { and } \mathfrak{e}_{6(2)}
$$

Write $\mathrm{D}^{1}=\mathrm{D} \cup\{$ lowest root\}. By ignoring the lowest root, 1, Table 1] implies that the painted diagram on $\mathrm{D}^{1}$ is equivalent to its diagram symmetry.

$$
\mathfrak{e}_{7(-5)}
$$

The Dynkin diagram of $\mathfrak{g}=E_{7}$ has no nontrivial symmetry, so $\operatorname{int}(\mathfrak{g})=\operatorname{aut}(\mathfrak{g})$.

Proposition 10.2 does not apply to $\mathrm{D}^{2}$ because its vertices $\alpha$ belong to $\mathfrak{h}^{*}$ instead of $\tilde{\mathfrak{h}}^{*}$, so $\left\{r_{\alpha}\right\}_{\alpha}$ do not generate the Weyl group of $\mathfrak{g}$. However, Figure 3 has nontrivial diagram symmetries only on $\mathfrak{s l}(p, \mathbb{H})$ and $\mathfrak{s o}(2 p+1,2 q+1)$, hence we can treat them individually. The idea is to "unfold" $\mathrm{D}^{2}$ to recover the original Dynkin diagram.

$$
\mathfrak{s l}(p, \mathbb{H})
$$

The complexification of $\mathfrak{s l}(p, \mathbb{H})$ is $\mathfrak{g}=\mathfrak{s l}(2 p, \mathbb{C})$. Let $n=2 p$. Let $E_{i, j}$ be the elementary matrix with 1 at the $(i, j)$-entry and 0 elsewhere. Let $\tilde{\mathfrak{h}}$ be the Cartan subalgebra of $\mathfrak{g}$ given by the diagonal matrices. Its root system is $\Delta_{\mathfrak{g}}=\Delta(\mathfrak{g}, \tilde{\mathfrak{h}})=$ $\left\{e_{i, j} ; i, j=1, \ldots, n\right.$ and $\left.i \neq j\right\}$, where $e_{i, j}\left(E_{k, k}\right)=\delta_{i, k}-\delta_{j, k}$. Let the Dynkin diagram represent the simple system $\Pi_{\mathfrak{g}}=\left\{e_{i, i+1} ; i=1, \ldots, n-1\right\}$.

Let $f$ permute $\{1, \ldots, n\}$ by $f(1)=n, f(n)=1$ and $f(i)=i$ for other $i$. Then define $\sigma \in \operatorname{aut}(\mathfrak{g})$ by $\sigma E_{i, j}=E_{f(i), f(j)}$. Define $d \in \operatorname{aut}\left(\Delta_{\mathfrak{g}}\right)$ by $d e_{i, j}=e_{f(i), f(j)}$. Then $\sigma \mathfrak{g}_{\alpha}=\mathfrak{g}_{d \alpha}$. The effect of $d$ on $\Pi_{\mathfrak{g}}$ is shown by Figure 10(a).

Use the Cartan involution of $\mathfrak{s l}(p, \mathbb{H})$ whose extension to $\mathfrak{g}$ preserves $\tilde{\mathfrak{h}}$ and switches the simple root spaces of the upper and lower rows of Figure 10(a). Let $\mathfrak{s l}(p, \mathbb{H})=\mathfrak{k}_{0}+\mathfrak{p}_{0}$ be the corresponding Cartan decomposition. So $\mathfrak{h}=\tilde{\mathfrak{h}} \cap \mathfrak{k}$ is a Cartan subalgebra of $\mathfrak{k}$. By restricting $\Pi_{\mathfrak{g}} \subset \Delta_{\mathfrak{g}}$ to $\mathfrak{h}$, we obtain $\Pi_{\mathfrak{k}} \subset \Delta(\mathfrak{g}, \mathfrak{h})$. Here $\Pi_{\mathfrak{k}}$ is given by averaging the vertices in the upper and lower rows of Figure $10(\mathrm{a})$, for example $\frac{1}{2}\left(e_{1,2}+e_{n-1, n}\right) \in \Pi_{\mathfrak{k}}$. The vertices of the affine diagram $A_{n}^{2}$ 

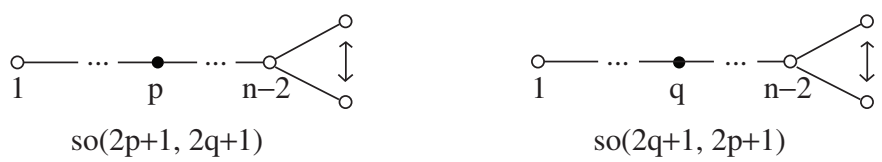

FIgURE 11

are $\Pi_{\mathfrak{k}} \cup\left\{\frac{1}{2}\left(e_{n, 2}+e_{n-1,1}\right)\right\}$, where $\frac{1}{2}\left(e_{n, 2}+e_{n-1,1}\right)$ is the lowest weight of the $\mathfrak{k}$ representation on $\mathfrak{p}$. This leads to Figure $10(\mathrm{~b})$. The $d$-action on $\Delta_{\mathfrak{g}}$ leads to a $d$-action on $\Delta(\mathfrak{g}, \mathfrak{h})$, and this is indicated in Figure 10(b).

Replace $\tilde{\mathfrak{h}}$ by another Cartan subalgebra $\tilde{\mathfrak{h}}^{\prime}$ of $\mathfrak{g}$, given by the span of

$$
\left\{E_{i, i}-E_{i+1, i+1}\right\}_{i=2}^{n-2} \cup\left\{E_{1, n}+E_{n, 1}, \frac{n-2}{2}\left(E_{1,1}+E_{n, n}\right)-\sum_{i=2}^{n-1} E_{i, i}\right\} .
$$

Then $\sigma$ fixes $\tilde{\mathfrak{h}}^{\prime}$, namely $\tilde{\mathfrak{h}}^{\prime} \subset \mathfrak{g}^{\sigma}$. By Proposition 10.1 $\sigma \in \operatorname{int}(\mathfrak{g})$. So the additional painted diagram resulting from the diagram symmetry $d$ in Figure 10(b) does not contribute to an extra inner isomorphic class of real form.

$$
\mathfrak{s o}(2 p+1,2 q+1)
$$

The Lie algebras $\mathfrak{s o}(2 p+1,2 q+1)$ and $\mathfrak{s o}(2 q+1,2 p+1)$ are isomorphic, and their painted diagrams are symmetric to each other. We unfold the affine diagram $D_{n}^{2}$

and draw their Vogan diagrams (Theorem 4.1) on $D_{n}$ directly as shown by Figure 11 .

By [1, Table 2], there is a sequence of $\left\{F_{\alpha}\right\}$ which transform the first diagram to the second. By Proposition 10.2 $\mathfrak{s o}(2 p+1,2 q+1)$ and $\mathfrak{s o}(2 q+1,2 p+1)$ are inner isomorphic.

\section{ACKNOWLEDGEMENTS}

The author is grateful to R. Sjamaar and D. Vogan for their helpful comments, and also to J. S. Huang and F. H. Zhu for discussions and hospitality at the Chern Institute of Mathematics.

\section{REFERENCES}

[1] M. K. Chuah and C. C. Hu, Equivalence classes of Vogan diagrams, J. Algebra 279 (2004), 22-37. MR2078384 (2005g:17021)

[2] M. K. Chuah and J. S. Huang, Double Vogan diagrams and semisimple symmetric spaces, Trans. Amer. Math. Soc. 362 (2010), 1721-1750. MR2574875 (2011a:17016)

[3] F. R. Gantmacher, Canonical representation of automorphisms of a complex semi-simple Lie group, Rec. Math. (Moscou) 5(47) (1939), 101-146. MR0000998(1:163d)

[4] S. Helgason, Differential Geometry, Lie Groups, and Symmetric Spaces, Graduate Studies in Math. vol. 34, Amer. Math. Soc., Providence 2001. MR.1834454 (2002b:53081)

[5] J. E. Humphreys, Introduction to Lie Algebras and Representation Theory, Springer-Verlag, New York 1972. MR0323842(48:2197)

[6] V. G. Kac, Automorphisms of finite order of semisimple Lie algebras, Funkcional Anal. $i$ Prilozen 3 (1969), 94-96. MR0251091 (40:4322)

[7] V. G. Kac, Infinite Dimensional Lie Algebras, 3rd. ed., Cambridge Univ. Press, Cambridge 1990. MR1104219 (92k:17038)

[8] A. W. Knapp, Lie Groups beyond an Introduction, 2nd. ed., Progr. Math. vol. 140, Birkhäuser, Boston 2002. MR 1920389 (2003c:22001)

[9] F. Levstein, A classification of involutive automorphisms of an affine Kac-Moody Lie algebra, J. Algebra 114 (1988), 489-518. MR936987 (90g:17025) 
[10] G. D. Mostow, Self-adjoint groups, Annals of Math. 62 (1955), 44-55. MR0069830 (16:1088a)

[11] A. L. Onishchik and E. B. Vinberg, Lie Groups and Algebraic Groups, Springer-Verlag, Berlin, Heidelberg 1990. MR.1064110 (91g:22001)

[12] T. A. Springer, Linear Algebraic Groups, 2nd. ed., Progr. Math. vol. 9, Birkhäuser, Boston 1998. MR1642713 (99h:20075)

Department of Mathematics, National Tsing Hua University, Hsinchu, Taiwan

E-mail address: chuah@math.nthu.edu.tw 